\title{
Hard color-singlet exchange in dijet events in proton-proton collisions at $\sqrt{s}=13 \mathrm{TeV}$
}

\author{
A. M. Sirunyan et al. \\ (CMS Collaboration $)^{\dagger}$ \\ (TOTEM Collaboration) $)^{\ddagger}$
}

(Received 13 February 2021; accepted 20 May 2021; published 26 August 2021)

\begin{abstract}
Events where the two leading jets are separated by a pseudorapidity interval devoid of particle activity, known as jet-gap-jet events, are studied in proton-proton collisions at $\sqrt{s}=13 \mathrm{TeV}$. The signature is expected from hard color-singlet exchange. Each of the highest transverse momentum $\left(p_{\mathrm{T}}\right)$ jets must have $p_{\mathrm{T}}^{\text {jet }}>40 \mathrm{GeV}$ and pseudorapidity $1.4<\left|\eta^{\text {jet }}\right|<4.7$, with $\eta^{\text {jet } 1} \eta^{\text {jet2 }}<0$, where jet 1 and jet 2 are the leading and subleading jets in $p_{\mathrm{T}}$, respectively. The analysis is based on data collected by the CMS and TOTEM experiments during a low luminosity, high- $\beta^{*}$ run at the CERN LHC in 2015 , with an integrated luminosity of $0.66 \mathrm{pb}^{-1}$. Events with a low number of charged particles with $p_{\mathrm{T}}>0.2 \mathrm{GeV}$ in the interval $|\eta|<1$ between the jets are observed in excess of calculations that assume only color-exchange. The fraction of events produced via color-singlet exchange, $f_{\mathrm{CSE}}$, is measured as a function of $p_{\mathrm{T}}^{\mathrm{jet} 2}$, the pseudorapidity difference between the two leading jets, and the azimuthal angular separation between the two leading jets. The fraction $f_{\mathrm{CSE}}$ has values of $0.4-1.0 \%$. The results are compared with previous measurements and with predictions from perturbative quantum chromodynamics. In addition, the first study of jet-gap-jet events detected in association with an intact proton using a subsample of events with an integrated luminosity of $0.40 \mathrm{pb}^{-1}$ is presented. The intact protons are detected with the Roman pot detectors of the TOTEM experiment. The $f_{\mathrm{CSE}}$ in this sample is $2.91 \pm 0.70$ (stat $)_{-1.01}^{+1.08}$ (syst) times larger than that for inclusive dijet production in dijets with similar kinematics.
\end{abstract}

DOI: $10.1103 /$ PhysRevD.104.032009

\section{INTRODUCTION}

Quantum chromodynamics (QCD) is the established theory of strong interactions and it is especially successful at very short distances, where physical observables can be computed in a perturbative expansion in powers of the strong coupling, $\alpha_{\mathrm{S}}$. However, there remain corners of phase space where predictions from perturbative QCD (pQCD) have yet to be confirmed. One such kinematic region is the highenergy limit of strong interactions, which is particularly important for better understanding the initial state in hadronic collisions and for studies of high-energy scattering [1,2].

In $2 \rightarrow 2$ parton scattering, the high-energy limit of QCD is mathematically represented by $\hat{s} \gg-\hat{t} \gg \Lambda_{\mathrm{QCD}}^{2}$, where $\hat{s}$ is the square of the partonic center-of-mass energy, $\hat{t}$ is the square of the partonic four-momentum transfer, and $\Lambda_{\mathrm{QCD}}$ is

${ }^{*}$ Full author list given at the end of the article.

Published by the American Physical Society under the terms of the Creative Commons Attribution 4.0 International license. Further distribution of this work must maintain attribution to the author(s) and the published article's title, journal citation, and DOI. Funded by SCOAP ${ }^{3}$. the energy scale below which QCD becomes strongly coupled. In this limit, some powers of $\alpha_{\mathrm{S}}$ are multiplied by a large logarithm of $\hat{s}$ in the perturbative expansion, compensating for the smallness of $\alpha_{\mathrm{S}} \ll 1$ such that $\alpha_{\mathrm{S}} \ln (\hat{s} /|\hat{t}|) \lesssim 1$. Thus, the fixed-order perturbation theory approach is no longer valid. These logarithmically enhanced terms correspond to multiple-parton splittings that are strongly ordered in rapidity. The Balitsky-Fadin-KuraevLipatov (BFKL) evolution equation resums these terms to all orders in $\alpha_{\mathrm{S}}$ in the perturbative expansion [3-5], and its solutions are known up to next-to-leading logarithmic (NLL) accuracy [6,7]. In dijet production, the expected onset of BFKL dynamics is reached in configurations where the two jets are separated by a large rapidity interval. The BFKL radiation pattern is also expected to be important in the study of parton distribution functions (PDFs) of hadrons [3-5]. In this context, the high-energy limit of QCD corresponds to the regime of very small values of the parton momentum fraction $x$ at low momentum transfer. The resummation of $\ln (1 / x)$ terms to all orders in $\alpha_{\mathrm{S}}$ predicts a power-law growth of gluon densities at small $x$.

At the CERN LHC, dedicated studies of BFKL dynamics include measurements of azimuthal angular $(\phi)$ 
decorrelations between jets in forward-backward dijet configurations [8] and cross section measurements at large values of the rapidity difference between the jets $[9,10]$. Exclusive vector meson production at the LHC [11-17] can be treated within the BFKL framework, as discussed in Refs. $[18,19]$. Measurements of inclusive jet or multijet cross sections at different center-of-mass energies show no significant deviations from predictions based on the Dokshitzer-Gribov-Lipatov-Altarelli-Parisi (DGLAP) evolution equations [20-22], where parton emissions are strongly ordered in transverse momentum $\left(p_{\mathrm{T}}\right)$, distinct from the BFKL ordering in rapidity, over a large region of phase space [9,10,23-36]. State-of-the-art global PDF fits highlight the importance of including resummation of small $x$ terms to all orders in $\alpha_{\mathrm{S}}$ to describe inclusive deep inelastic scattering data collected by the DESY HERA experiments [37]. A lesson from these studies is that BFKL dynamical effects associated with multiple parton splittings are very difficult to separate from other effects predicted by higher-order corrections in $\mathrm{pQCD}$. More restrictive finalstate studies, where other effects expected from $\mathrm{pQCD}$ are suppressed, may provide clearer indications of BFKL dynamics.

A study of events is presented in proton-proton $(p p)$ collisions with two jets separated by a large pseudorapidity $(\eta)$ interval devoid of particle activity. These are known as Mueller-Tang jets [38] or jet-gap-jet events. The jet-gap-jet events in this study are observed with the CMS detector. Previous studies of jet-gap-jet events have been carried out by the H1 and ZEUS Collaborations in dijet photoproduction in electron-proton collisions at the DESY HERA $[39,40]$, by the CDF and D0 Collaborations in $p p$ collisions at center-of-mass energies $\sqrt{s}=0.63$ and $1.8 \mathrm{TeV}$ at the Fermilab Tevatron [41-46], and by CMS at $7 \mathrm{TeV}$ in $p p$ collisions at the CERN LHC [47]. The pseudorapidity gap is indicative of an underlying $t$-channel hard color-singlet exchange [48-51]. In the BFKL framework, hard colorsinglet exchange is described by $t$-channel two-gluon ladder exchange between the interacting partons, as shown in Fig. 1, where the color charge carried by the exchanged

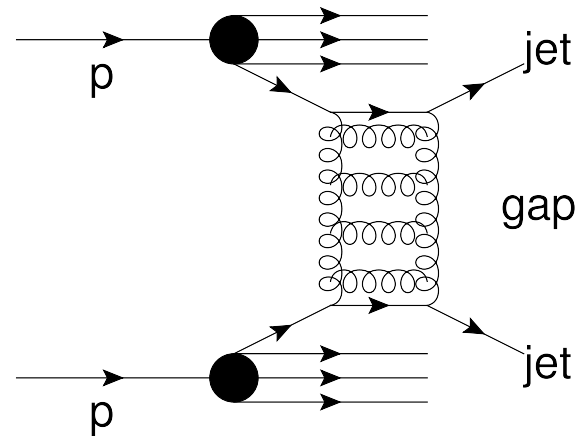

gluons cancel, leading to a suppression of particle production between the final-state jets. This is known as perturbative pomeron exchange [3-5]. Color-singlet exchange can occur in quark-quark, quark-gluon, and gluon-gluon scattering. Of these, gluon-gluon scattering is expected to be substantially favored as a result of the larger color charge of gluons [49-51]. In contrast, in most collisions that lead to dijet production, the net color charge exchange between partons results in final-state particle production over wide intervals of rapidity between the jets. These color-exchange dijet events are referred to in this paper as "background" events. Dynamical effects predicted by the DGLAP evolution equations are largely suppressed in events with pseudorapidity gaps, since the predicted dijet production rate is strongly reduced by way of a Sudakov form factor [48-51]. This factor, which accounts for the probability of having no additional parton emissions between the hard partons, is not necessary for BFKL pomeron exchange [38]. The ratio of jet-gap-jet yields to inclusive dijet yields is sensitive to dynamical effects predicted by the BFKL evolution equations, as first suggested in Ref. [38] and further studied in Refs. [52-56].

The presence of soft rescattering effects between partons and the proton remnants modify the visible cross section of jet-gap-jet events. These soft interactions can induce the production of particles in the $\eta$ interval that would otherwise be devoid of particles. This results in a reduction of the number of events identified as having a jet-gap-jet signature. This reduction is parametrized using a multiplicative factor known as the rapidity gap survival probability, $|\mathcal{S}|^{2}$. The survival probability is a process-dependent, nonperturbative quantity [48,57-61] that is expected to have values of the order of $|\mathcal{S}|^{2}=1-10 \%$ at LHC energies. This factor is often assumed to be largely independent of the dijet event kinematics [48], although some nonperturbative models, such as the soft color interactions (SCI) model $[53,56]$, suggest that this is not always the case. In particular, multiple-parton interactions (MPI) can further reduce the survival probability in dijet events with a central gap, as discussed in Refs. [53,56,62].

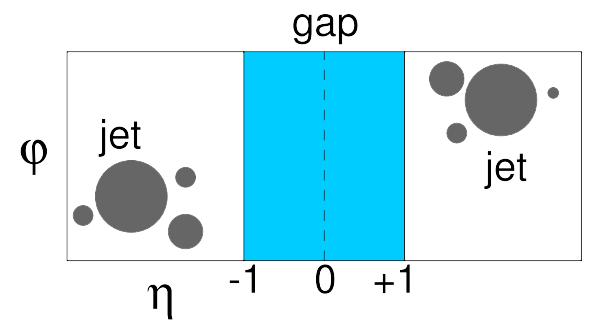

FIG. 1. Left: schematic diagram of a jet-gap-jet event by hard color-singlet exchange in $p p$ collisions. The lines following the protons represent the proton breakup. Right: jet-gap-jet event signature in the $\eta$ - $\phi$ plane. The filled circles represent final-state particles. The shaded rectangular area between the jets denotes the interval $|\eta|<1$ devoid of charged particles. 

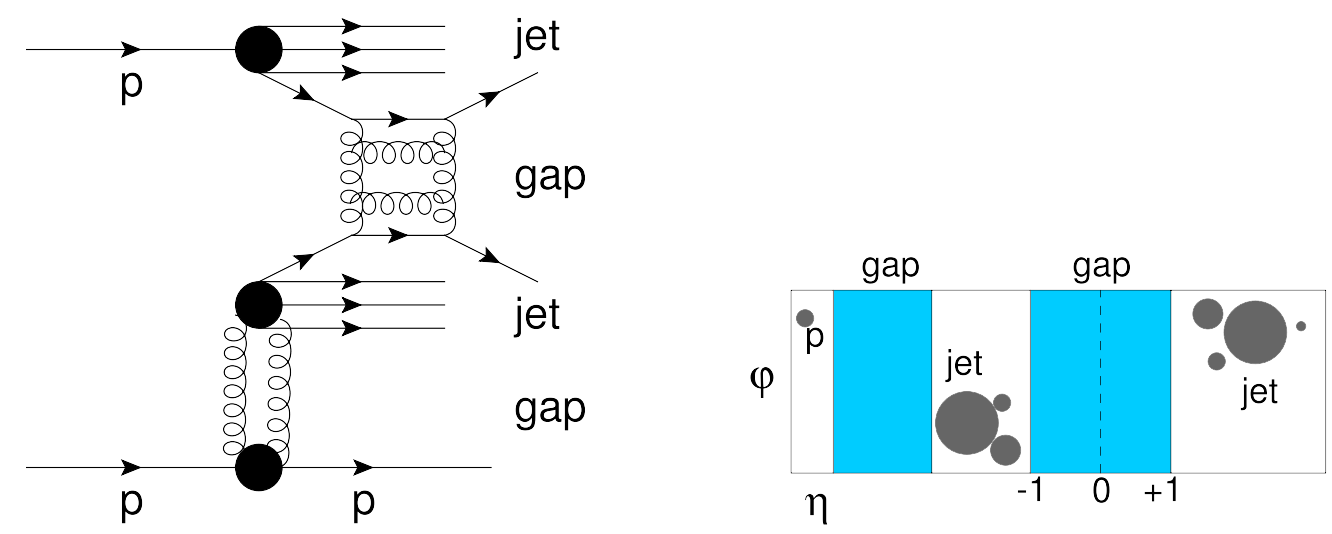

FIG. 2. Left: schematic diagram of a jet-gap-jet event by hard color-singlet exchange with an intact proton in $p p$ collisions. The jetgap-jet is reconstructed in the CMS detector, while the intact proton is detected with one of the forward proton spectrometers of the TOTEM experiment. Right: proton-gap-jet-gap-jet event signature in the $\eta-\phi$ plane. The filled circles represent final-state particles. The shaded rectangular areas denote the central gap region $|\eta|<1$ devoid of charged particles and the forward gap that is inferred from the forward proton detection.

Soft rescattering effects can be suppressed in processes where one or both of the colliding protons remain intact after the interaction, such as in single- or central-diffractive dijet processes or in dijet photoproduction. These can be used to better separate events with a central gap between the jets, as discussed in Ref. [63]. Hence, parallel to the study of jet-gap-jet events in inclusive dijet production, a study of jet-gap-jet events with an intact proton, as shown in Fig. 2, is also presented. Although no forward rapidity gap is required in the analysis, these events are referred to as "proton-gap-jet-gap-jet" throughout the paper, where the forward rapidity gap signature is inferred from the detection of the intact proton. This part of the analysis uses a subset of dijet events that, in addition, have intact protons detected with the forward proton spectrometers of the TOTEM experiment [64]. This diffractive event topology has not been previously measured.

The present study is based on low instantaneous luminosity data collected in $p p$ collisions at $\sqrt{s}=13 \mathrm{TeV}$ by the CMS and TOTEM experiments at the CERN LHC. These data were recorded with special LHC optics settings, $\beta^{*}=90 \mathrm{~m}$, where $\beta^{*}$ is the betatron amplitude function at the interaction point [65]. Data were recorded by CMS with an integrated luminosity of $0.66 \mathrm{pb}^{-1}$; a subset of the data with $0.40 \mathrm{pb}^{-1}$ was collected jointly with the TOTEM experiment. The present analysis uses a similar event selection and central gap definition as the previous measurement by CMS at $7 \mathrm{TeV}$ [47]. Each of the two highest $p_{\mathrm{T}}$ jets must have $p_{\mathrm{T}}^{\text {jet }}>40 \mathrm{GeV}$ and $1.4<\left|\eta^{\mathrm{jet}}\right|<4.7$, and they must be in opposite hemispheres of the detector $\eta^{\mathrm{jet} 1} \eta^{\mathrm{jet} 2}<0$, where jet 1 and jet2 denote the leading and subleading jets in $p_{\mathrm{T}}$, respectively. The charged particle multiplicity ( $N_{\text {tracks }}$ ) in the interval $|\eta|<1$ between the two leading jets, where each charged particle must have $p_{\mathrm{T}}>200 \mathrm{MeV}$, is used to isolate color-singlet exchange dijet events from color-exchange dijet events. Jet-gap-jet events due to color-singlet exchange are characterized by a sharp excess at the lowest $N_{\text {tracks }}$ values above the expected contribution of color-exchange dijet events. The increase in $\sqrt{s}$ to $13 \mathrm{TeV}$ provides improved conditions to study the hard color-singlet exchange process in an unexplored region of phase space. The increased sample size relative to the previous analysis at $7 \mathrm{TeV}$ allows finer binning in the kinematic variables of interest and an improved precision in the determination of the fraction of dijet events produced via hard color-singlet exchange. Furthermore, the analysis based on CMS and TOTEM data provides a first investigation of dijet events with a central gap and an intact proton. This analysis can elucidate the role of soft parton exchanges in the creation and destruction mechanisms of pseudorapidity gaps in strong interactions [63]. The intact protons in the analysis have a fractional momentum loss $(\xi)$ of up to $20 \%$, with values of the square of the fourmomentum transfer at the proton vertex $(t)$ in the range between -4 and $-0.025 \mathrm{GeV}^{2}$.

The paper is organized as follows. The CMS and TOTEM detectors are introduced in Sec. II. The data sample used in the analysis is described in Sec. III. The event selection requirements are presented in Sec. IV. The central pseudorapidity gap and observable definitions are discussed in Secs. V and VI, respectively. Section VII gives a description of the background treatment used in the analysis. The systematic uncertainties are detailed in Sec. VIII. The results of the paper are shown in Sec. IX. A summary of the paper is found in Sec. X.

\section{THE CMS AND TOTEM DETECTORS}

The central feature of the CMS apparatus is a superconducting solenoid of $6 \mathrm{~m}$ internal diameter, providing a magnetic field of $3.8 \mathrm{~T}$. Within the solenoid volume are a silicon pixel and strip tracker, a lead tungstate crystal electromagnetic calorimeter (ECAL), and a brass and 
scintillator hadron calorimeter (HCAL), each composed of a barrel and two endcap sections. Forward calorimeters extend the $\eta$ coverage provided by the barrel and endcap detectors. Muons are detected in gas-ionization chambers embedded in the steel flux-return yoke outside the solenoid.

The silicon tracker measures charged particles within the range $|\eta|<2.5$. It consists of 1440 silicon pixel and 15148 silicon strip detector modules. For nonisolated particles with $1<p_{\mathrm{T}}<10 \mathrm{GeV}$ and $|\eta|<1.4$, the track resolutions are typically $1.5 \%$ in $p_{\mathrm{T}}$ and $25-90(45-150) \mu \mathrm{m}$ in the transverse (longitudinal) impact parameter [66].

The particle-flow (PF) algorithm [67] aims to reconstruct and identify each individual particle (physics-object) in an event, with an optimized combination of information from the various elements of the CMS detector. The energy of photons is obtained from the ECAL measurement. The energy of electrons is determined from a combination of the electron momentum at the primary interaction vertex as determined by the tracker, the energy of the corresponding ECAL cluster, and the energy sum of all bremsstrahlung photons spatially compatible with originating from the electron track. The energy of muons is obtained from the curvature of the corresponding track. The energy of charged hadrons is determined from a combination of their momentum measured in the tracker and the matching ECAL and HCAL energy deposits, corrected for the response function of the calorimeters to hadronic showers. Finally, the energy of neutral hadrons is obtained from the corresponding corrected ECAL and HCAL energies.

Tracks are reconstructed with the standard iterative algorithm of CMS [66]. To reduce the misidentification rate, tracks are required to pass standard CMS quality criteria, referred to as high-purity criteria. High-purity tracks satisfy requirements on the number of hits and the $\chi^{2}$ of the track-fit. The requirements are functions of the charged particle track $p_{\mathrm{T}}$ and $\eta$, as well as the number of layers with a hit. A more detailed discussion of the combinatorial track finding algorithm and the definition of high-purity tracks is reported in Ref. [66]. The reconstruction efficiency for high-purity tracks is about $75 \%$ with $p_{\mathrm{T}}>200 \mathrm{MeV}$. The candidate vertex with the largest value of summed physics-object $p_{\mathrm{T}}^{2}$ is taken to be the primary $p p$ interaction vertex. In the vertex fit, each track is assigned a weight between 0 and 1 , which reflects the likelihood that it genuinely belongs to the vertex. The number of degrees of freedom in the fit is strongly correlated with the number of tracks arising from the interaction region, as described in Ref. [66].

The jets are clustered using the infrared- and collinearsafe anti- $k_{\mathrm{T}}$ algorithm $[68,69]$, with a distance parameter of $R=0.4$. The clustering is performed with the FastJet package [69]. The key feature of the anti- $k_{\mathrm{T}}$ algorithm is the resilience of the jet boundary with respect to soft radiation. This leads to cone-shaped hard jets. The jet momentum is determined as the vector sum of all particle momenta in the jet. The simulations show the CMS detector response is within 5-10\% of the true hadron-level momentum over a wide range of the jet $p_{\mathrm{T}}$ and $\eta$. Jet energy corrections are derived from simulation to bring, on average, the measured jet energies to the known energies at the generator level [70]. In situ measurements of the momentum balance in dijet, photon + jet, $Z+$ jet, and multijet events are used to correct any residual differences in the jet energy scale in data and simulation [70]. The jet energy resolution typically amounts to $15 \%$ at $10 \mathrm{GeV}, 8 \%$ at $100 \mathrm{GeV}$, and $4 \%$ at $1 \mathrm{TeV}$. A more detailed description of the CMS detector, together with a definition of the coordinate system used and the relevant kinematic variables, is described in Ref. [71].

The proton spectrometer of the TOTEM experiment consists of two sets of telescopes, known as Roman pot (RP) stations [64] that are located close to the beamline. The arms are referred to as sectors 45 and 56 for positive and negative $\eta$, respectively. An RP that contains silicon strip detectors can approach the LHC beam to a distance of a few millimeters without affecting the LHC operation [64]. The RPs are used to detect protons deflected at scattering angles of only a few microradians relative to the beam. During the 2015 special run, there were two RP stations operating in each sector located at $\pm 210 \mathrm{~m}$ and $\pm 220 \mathrm{~m}$ relative to the interaction point. The configuration during 2015 is depicted in Fig. 3. The station at $210 \mathrm{~m}$ has one unit of RPs, while the station at $220 \mathrm{~m}$ has two units of RPs.

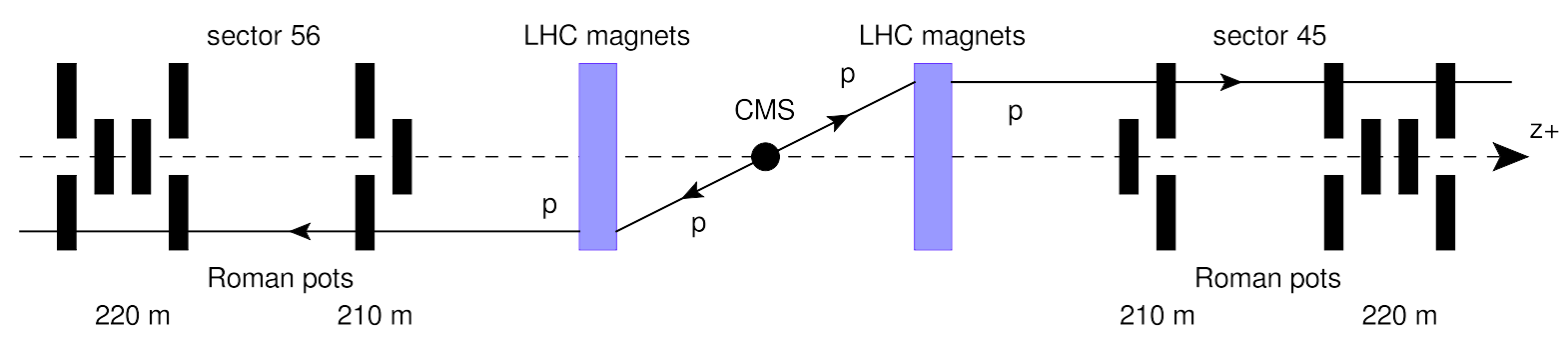

FIG. 3. Profile schematic of the CMS-TOTEM detector configuration during the 2015 run. The horizontal dashed line represents the beamline. The CMS detector is denoted by the filled circle in the center. The intact proton(s) are transported via the accelerator magnetic fields (violet light rectangles), eventually passing through the silicon detectors housed in the Roman pots (black dark rectangles) of the TOTEM experiment. Sectors 45 and 56 are located in the positive and negative $\eta$ regions in the CMS coordinate system, respectively. 
Each unit has three RPs: one located above ("top"), one below ("bottom"), and one to one side ("horizontal") of the LHC beam [64]. Before being detected, the trajectories of protons that have lost a small amount of their original momentum slightly deviate from the beam trajectory, with the deviation dependent on the momentum of the proton. The intact proton kinematics are reconstructed after modeling the transport of the protons from the interaction point to the RP location $[64,72]$. With the $\beta^{*}=90 \mathrm{~m}$ conditions, small horizontal displacements of the forward proton tracks at the RPs are directly proportional to $\xi$. The detection of the forward protons also enables the reconstruction of $t$, which is related to the horizontal and vertical scattering angles of the proton track at the RPs $[73,74]$. The resolution in $\xi$ is 0.008 for $\xi \approx 0$ and 0.002 for $\xi=0.2$ [73]. The RPs are aligned following the standard techniques developed by the TOTEM Collaboration [73]. The TOTEM detector is described in Refs. [64,73].

\section{DATA SAMPLE AND TRIGGER SELECTION}

The $p p$ collision data used in this analysis were collected in a combined special run by the CMS and TOTEM experiments in 2015 at $\sqrt{s}=13 \mathrm{TeV}$, when the LHC operated in a mode with low probability of overlapping $p p$ interactions in the same bunch crossing (pileup). With $\beta^{*}=$ $90 \mathrm{~m}$ optics at the interaction point of CMS, there were about $0.05-0.10$ pileup interactions per event. Events were selected by trigger signals delivered simultaneously to the CMS and TOTEM detectors. The CMS orbit-counter reset signal, delivered to the TOTEM electronics at the start of the run, assures the time synchronization of the two experiments. The samples were combined offline by matching bunch crossing and orbit numbers, as in the previous CMS and TOTEM combined run at $\sqrt{s}=8 \mathrm{TeV}$ [75]. Since CMS and TOTEM collected data simultaneously for a fraction of the special run, the integrated luminosity for the CMS-TOTEM sample corresponds to $0.40 \mathrm{pb}^{-1}$. The data were collected with an unprescaled inclusive dijet trigger. This trigger requires at least two leading jets (jet1, jet2), both with $p_{\mathrm{T}}>32 \mathrm{GeV}$ with $|\eta|<5$ [76]. The trigger is about $85 \%$ efficient for $p_{\mathrm{T}}^{\mathrm{jet} 2}=40 \mathrm{GeV}$, and is fully efficient at $p_{\mathrm{T}}^{\text {jet }}>55 \mathrm{GeV}$, as measured with dijet events in a zero-bias sample collected using a random trigger in the presence of nonempty bunch crossings. Trigger efficiency effects largely cancel in the ratio of yields of events with a central gap, $f_{\mathrm{CSE}}$, the main observable measured in this analysis, which is described in Sec. VI. Thus, no efficiency correction is applied in the analysis. A subset of events of the zero-bias sample that contains forward proton information collected by the TOTEM experiment is used for systematic checks in the analysis.

\section{EVENT SELECTION}

\section{A. Dijet event selection}

The following selection requirements are used for the study of jet-gap-jet events within inclusive dijet events as well as for the analysis of jet-gap-jet events with an intact proton:

(i) Each of the two leading jets is required to have $p_{\mathrm{T}}^{\text {jet }}>40 \mathrm{GeV}$. This selection maximizes the number of dijet events considered in the analysis, while ensuring high dijet reconstruction efficiency. The phase space explored in the present analysis is similar to that studied in the previous CMS measurement at $7 \mathrm{TeV}$ [47]. There are no requirements on additional jets that may be produced in the collision.

(ii) The two leading jets are measured in opposite hemispheres of the CMS detector, $\eta^{\text {jet1 }} \eta^{\text {jet2 }}<0$, and must have $1.4<\left|\eta^{\text {jet }}\right|<4.7$. This selection favors the phase space region for production of jet-gap-jet events. Jets are reconstructed with the anti- $k_{\mathrm{T}}$ algorithm distance parameter $R=0.4$ and the adopted jet $\eta$ range thus locates the jets at least one unit of $R$ away from the $|\eta|<1$ region used to extract the multiplicity of charged particles.

(iii) The number of reconstructed primary vertices in the event is required to be at most one. This requirement is used to reject residual pileup interactions. For this analysis, a primary vertex is kept if it has at least two degrees of freedom as defined in Ref. [66]. Keeping events with no primary vertex retains forward-backward dijet configurations that have too few tracks to establish a primary vertex, as is likely for the jet-gapjet topology.

(iv) The primary vertex, if present, is required to be located within a longitudinal distance of $24 \mathrm{~cm}$ of the nominal interaction point of CMS.

There were 362915 dijet events satisfying these selection requirements.

\section{B. Intact proton selection}

For the study of jet-gap-jet events with an intact proton (proton-gap-jet-gap-jet), in addition to the dijet event selection described in Sec. IVA, the following selection requirements on the protons reconstructed in the RPs are also applied:

(i) At least one proton must be detected in either sector 45 or 56 RP stations.

(ii) The proton track must cross at least two overlapping RP units (e.g., top-top, bottom-bottom), to ensure quality proton reconstruction.

(iii) The $\xi$ reconstructed with the $\mathrm{RP}\left(\xi_{p}(\mathrm{RP})\right)$ must have values of $\xi_{p}(\mathrm{RP})<0.2$ and $t$ must have values of $-4<t<-0.025 \mathrm{GeV}^{2}$. These bounds are based on acceptance studies of the RPs. 
(iv) The proton track impact location at the RP must satisfy the fiducial selection requirements $8<$ $|y(\mathrm{RP})|<30 \mathrm{~mm}$ and $0<x(\mathrm{RP})<20 \mathrm{~mm}$ for vertical RPs, and $|y(\mathrm{RP})|<25 \mathrm{~mm}$ and $7<x(\mathrm{RP})<$ $25 \mathrm{~mm}$ for horizontal RPs, where $x(\mathrm{RP})$ and $y(\mathrm{RP})$ denote the horizontal and vertical coordinates of the tracks in the plane transverse to the beamline at the $\mathrm{RP}$. The beam position is at $x(\mathrm{RP})=y(\mathrm{RP})=0$. This selection requirement ensures good proton reconstruction efficiency and acceptance within the RPs, and is based on acceptance studies of the RPs.

For the final selection requirement, the main goal is the removal of beam background events, which consist mostly of dijet events paired with uncorrelated beam halo particles or protons from residual pileup interactions. The beam halo is created by the interaction of beam particles with the collimation instrumentation or with residual gas in the vacuum chamber. To suppress these contributions, the following condition is applied:

(i) Events must satisfy $\xi_{p}(\mathrm{PF})-\xi_{p}(\mathrm{RP})<0$, where $\xi_{p}(\mathrm{PF})=\sum_{i}\left(E^{i} \pm p_{z}^{i}\right) / \sqrt{s}$ is the fractional momentum loss of the proton calculated with the PF candidates of CMS. Here, $E^{i}$ and $p_{z}^{i}$ are the energy and longitudinal momentum of the $i$ th PF candidate in the event, respectively. The positive or negative sign in the sum corresponds to the scattered proton moving toward the positive or negative $z$ direction in the CMS coordinate system, corresponding to the sector 45 or 56 directions, respectively. The PF candidates considered in the analysis have $|\eta|<5.2$.

For the calculation of $\xi_{p}(\mathrm{PF})$, charged $\mathrm{PF}$ objects in $|\eta|<2.5$ with a minimum $p_{\mathrm{T}}>200 \mathrm{MeV}$ are considered. A minimum energy of 1.7 and $1.2 \mathrm{GeV}$ is used at $|\eta|<1.4$ for neutral hadrons and photon candidates of the PF candidate collection, respectively. For neutral hadrons and photon candidates in the region $1.4<|\eta|<2.5$, a respective minimum energy of 3.25 and $3.00 \mathrm{GeV}$ is used. In the forward region, $2.5<|\eta|<5.2$, PF candidates with an energy greater than $5 \mathrm{GeV}$ are selected. These $\eta$-dependent energy thresholds were optimized based on zero-bias data collected during the same run conditions as in the dijet data sample. This follows from a similar procedure used in the $7 \mathrm{TeV}$ single-diffractive dijet analysis by CMS [77] and the $8 \mathrm{TeV}$ CMS-TOTEM study on diffractive dijet production [75].

Ideally, it is expected that the fractional momentum loss reconstructed with the central detector or the forward proton detectors should be the same, i.e., $\xi_{p}(\mathrm{PF})=\xi_{p}(\mathrm{RP})$. However, because of reconstruction inefficiencies and acceptance limitations of the CMS detector, and the use of energy thresholds applied for each PF candidate reconstructed in CMS, these events satisfy instead the inequality $\xi_{p}(\mathrm{PF})-\xi_{p}(\mathrm{RP})<0$, i.e., the fractional momentum loss is underestimated by the CMS detector. Therefore, the region
$\xi_{p}(\mathrm{PF})-\xi_{p}(\mathrm{RP})>0$ is dominated by events with uncorrelated forward protons that arise from pileup interactions or beam halo activity, since they do not have to satisfy the same bounds as the physical diffractive events. There is a residual contribution from these events in $\xi_{p}(\mathrm{PF})-\xi_{p}(\mathrm{RP})<0$, which is subtracted from the data, as explained in Sec. VII B. The same selection requirement that targets the suppression of beam background contributions was also used in the measurement of single-diffractive dijet production at $\sqrt{s}=$ $8 \mathrm{TeV}$ by the CMS and TOTEM Collaborations [75].

There are 341 and 336 events satisfying the dijet and intact proton selection requirements in sectors 45 and 56, respectively.

\section{CENTRAL GAP BETWEEN THE JETS}

Jet-gap-jet events arising from color-singlet exchange cannot be identified on an event-by-event basis since colorexchange dijet events can also have central gaps through fluctuations in the particle activity between the two jets. Nevertheless, the color-singlet exchange dijet process is expected to lead to an increase in the number of dijet events at the lowest particle multiplicities over those expected to arise from color exchange.

In this analysis, the charged particle activity between the two leading jets is used to characterize the pseudorapidity gap between the jets. The multiplicity of charged particles, $N_{\text {tracks }}$, is defined as the number of reconstructed charged particle tracks between the two leading jets, where each charged particle is in the interval $|\eta|<1$ and has $p_{\mathrm{T}}>200 \mathrm{MeV}$. The measured relative $p_{\mathrm{T}}$ uncertainty of each charged particle is required to be smaller than $10 \%$; this reduces the contribution from badly reconstructed or low-quality tracks. Reconstructed charged particle tracks satisfy the high-purity criteria of CMS described in Ref. [66]. The central gap is defined as the absence of charged particle production for $|\eta|<1$, which is the same definition used in the previous study at $\sqrt{s}=7 \mathrm{TeV}$ [47].

The fixed pseudorapidity gap region $|\eta|<1$ is the same as that employed in previous measurements by the CDF, D0, and CMS Collaborations [42,43,45-47], which facilitates the comparison of the findings of the present analysis with those previously reported at lower $\sqrt{s}$. Since $\left|\eta^{\text {jet } 1,2}\right|>$ 1.4 and $\eta^{\text {jet1 }} \eta^{\text {jet2 }}<0$, the separation between the jet axes starts at about 3 units in $\eta$, which is the minimum gap width typically used in studies of diffractive reactions in high energy physics. At the same time, the $\eta$ region is large enough to allow for a controlled subtraction of colorexchange dijet contributions. There are 1650 jet-gap-jet candidate events with $N_{\text {tracks }}=0$ in the sample. Although it is expected that jet-gap-jet events should only yield $N_{\text {tracks }}=0$, events with multiplicities up to $N_{\text {tracks }}=2$ occur in the signal when jet constituents are emitted at wide angles into the $|\eta|<1$ region, as discussed in Sec. VII. 
For a central gap definition based on neutral hadrons or photons, the corresponding $p_{\mathrm{T}}$ thresholds cannot be lowered to the $200 \mathrm{MeV}$ scale as with charged particle tracks. The noise level $p_{\mathrm{T}}$ thresholds are 0.5 and $2 \mathrm{GeV}$ for photons and neutral particles at central pseudorapidities, respectively, which leads to a looser definition of an $\eta$ interval devoid of particle activity. Consequently, neutral hadrons and photons are not used in the definition of the central gap in this analysis.

When an intact proton is included, the same definition of the central gap between the jets described above is used. The forward gap is inferred from the direct detection of the scattered proton, i.e., no calorimeter-based rapidity gap is applied. A total of 11 events are found with $N_{\text {tracks }}=0$ for dijet events with an intact proton.

Features of the dijet sample enriched in jet-gap-jet events are presented in Fig. 4. Events with $N_{\text {tracks }}=0$ are dominated by jet-gap-jet events, whereas events with

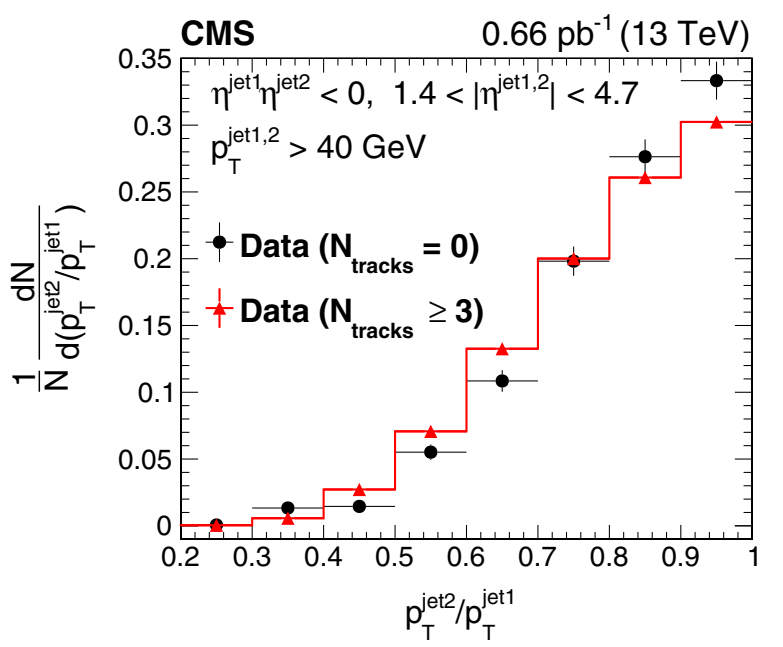

$N_{\text {tracks }} \geq 3$ are dominated by color-exchange dijet events. Jet-gap-jet candidates have the two leading jets strongly correlated in their transverse momenta, as shown in the upper panels of Fig. 4. This is characteristic of the nearly elastic parton-parton hard scattering process that initiated the jet production. The jet multiplicity, where each extra jet has $p_{\mathrm{T}}{ }^{\text {extra-jet }}>15 \mathrm{GeV}$ and $\left|\eta^{\text {extra-jet }}\right|<4.7$, is shown in the lower panel of Fig. 4. Most of the jet-gap-jet event candidates consist of two-jet events, whereas colorexchange dijet events feature multiple jets.

\section{COLOR-SINGLET EXCHANGE FRACTION}

Ideally, hard color-singlet exchange events should have only $N_{\text {tracks }}=0$. Occasionally, however, charged particles created during the fragmentation process are produced at large angles with respect to the jet boundary, such that they are emitted into the $|\eta|<1$ region. This leads to spillage of
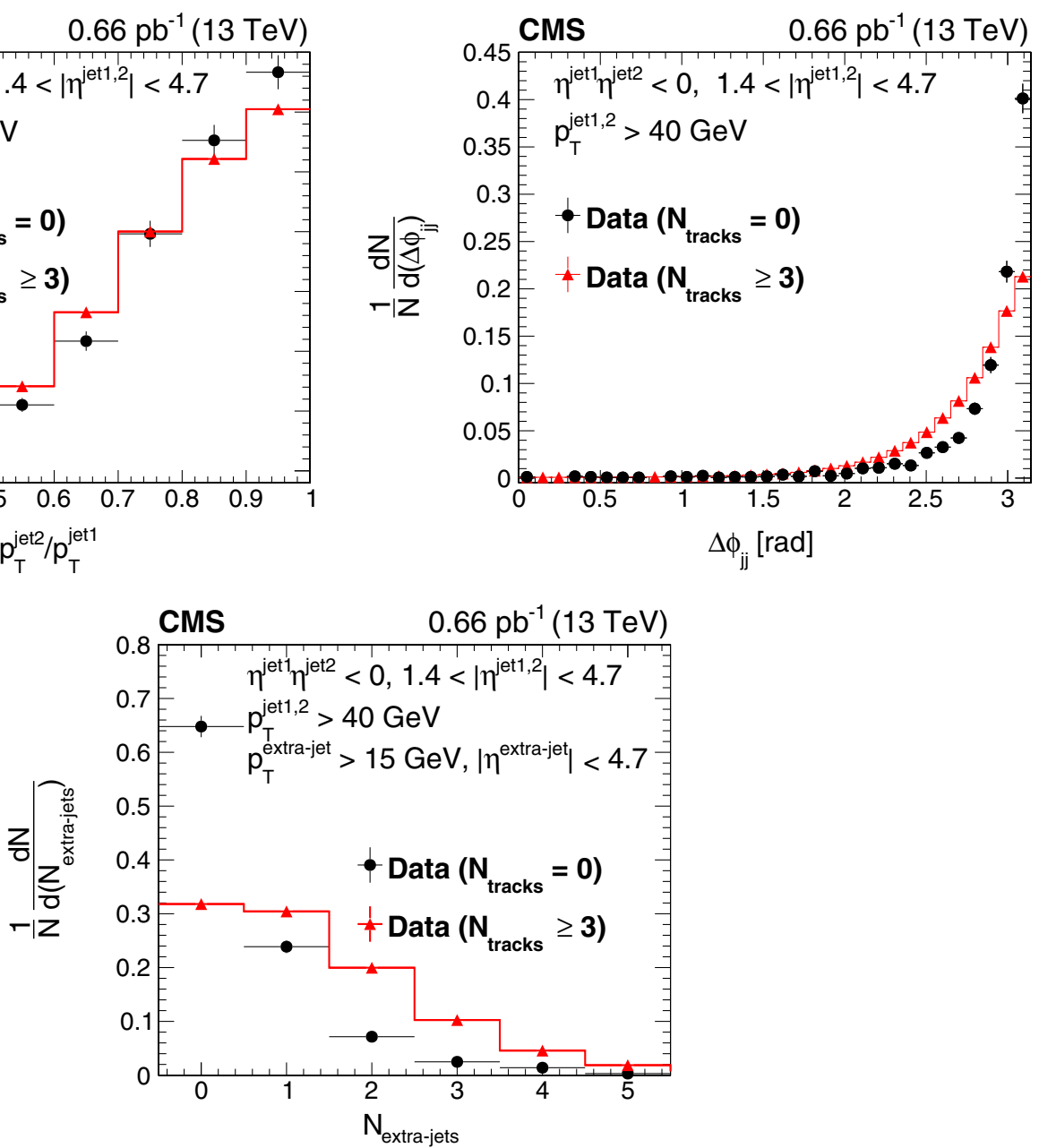

FIG. 4. Distributions of the ratio of the subleading jet to leading jet transverse momenta $p_{\mathrm{T}}^{\text {jet } 2} / p_{\mathrm{T}}^{\text {jet } 1}$ (left panel), the azimuthal angular separation between the two leading jets $\Delta \phi_{\mathrm{jj}}$ (right panel), and the number of additional jets $N_{\text {extra-jets }}$ with $p_{\mathrm{T}}{ }^{\text {extra-jet }}>15 \mathrm{GeV}$ (lower panel), for jet-gap-jet candidates with $N_{\text {tracks }}=0$ in $|\eta|<1$ (black circle) and color-exchange dijet candidates $N_{\text {tracks }} \geq 3$ in $|\eta|<1$ (red triangle). The vertical bars represent the statistical uncertainties, which are smaller than the marker for some data points. The horizontal bars represent the bin width. The distributions are normalized to unity. 
the color-singlet exchange signal events into the neighboring multiplicity counts. Therefore, the jet-gap-jet contributions are extracted for multiplicities up to $N_{\text {tracks }}=2$. The integration interval $N_{\text {tracks }}<3$ is optimized based on the background studies described in Sec. VII, where the excess of events over the expected number of colorexchange dijet events at low multiplicities is observed to stabilize, within the statistical uncertainties, at $N_{\text {tracks }}<3$.

The number of dijet events with $N_{\text {tracks }}<3$ is denoted by $N^{\mathrm{F}}$, the number of dijet events with no underlying colorsinglet exchange with $N_{\text {tracks }}<3$ by $N_{\text {non-CSE}}^{\mathrm{F}}$, and the total number of dijet events by $N$. The yields $N^{\mathrm{F}}$ and $N$ are extracted directly via event counting, whereas $N_{\text {non-CSE }}^{\mathrm{F}}$ requires modeling of color-exchange dijet events, which is discussed in Section VII.

The fraction of color-singlet exchange dijet events is given by

$$
f_{\mathrm{CSE}}=\frac{N^{\mathrm{F}}-N_{\text {non-CSE }}^{\mathrm{F}}}{N},
$$

and is measured as a function of kinematic variables of interest. Because $f_{\mathrm{CSE}}$ is a ratio of yields, jet reconstruction uncertainties approximately cancel. The $f_{\mathrm{CSE}}$ fraction can be measured as a function of:

(i) The pseudorapidity separation of the two leading jets, $\Delta \eta_{\mathrm{jj}} \equiv|\eta\rangle^{\mathrm{jet} 1}-\eta^{\mathrm{jet} 2} \mid$.

(ii) The subleading jet transverse momentum, $p_{\mathrm{T}}^{\mathrm{jet} 2}$.

(iii) The azimuthal angular separation between the two leading jets, $\Delta \phi_{\mathrm{jj}} \equiv\left|\phi^{\mathrm{jet} 1}-\phi^{\mathrm{jet} 2}\right|$.

The fraction $f_{\mathrm{CSE}}$, measured as a function of $\Delta \eta_{\mathrm{jj}}$, is particularly sensitive to predictions based on perturbative calculations within the BFKL framework [52-56], since it is directly related with the resummation of large logarithms of energy. The fraction $f_{\mathrm{CSE}}$, as a function of $p_{\mathrm{T}}^{\text {jet2 }}$, can be compared with phenomenology studies that predict a weak dependence of this fraction on $p_{\mathrm{T}}^{\text {jet} 2}$ based on BFKL calculations [52-55]. This $p_{\mathrm{T}}^{\mathrm{jet} 2}$ dependence also compares better with previous measurements by D0 $[42,43]$ and CMS [47]. The fraction $f_{\mathrm{CSE}}$, as a function of $\Delta \phi_{\mathrm{jj}}$, is sensitive to deviations from the back-to-back topology of jet-gap-jet events caused by higher-order perturbative QCD corrections, e.g., those induced by higher order corrections to the impact factors, which are related to the coupling of the perturbative pomeron to quarks and gluons [78,79]. The $f_{\text {CSE }}$ is extracted in bins of the kinematic variables of interest with ranges specified in Tables II-IV of Sec. IX.

For the measurement with intact protons, $f_{\mathrm{CSE}}$ is the ratio of the number of proton-gap-jet-gap-jet events to the number of standard diffractive dijet events. In this case, signal events are extracted in the first two multiplicity bins, $N_{\text {tracks }}<2$. The integration region of $N_{\text {tracks }}<2$ is optimized based on the background studies described in Sec. VII B, where an excess of events over background expectations is observed up to $N_{\text {tracks }}<2$, and on the lower mean multiplicity found in data in events with intact protons. Because of the limited sample size, a measurement as a function of kinematic variables is not possible. Thus, the respective $f_{\mathrm{CSE}}$ is extracted using the entire sample of events with the intact proton.

\section{BACKGROUND TREATMENT}

Two independent, data-based techniques are used to describe the contribution of color-exchange dijet events in the lowest multiplicity bins. The first method relies on a data sample independent of the nominal sample, whereas the second method relies on a parametrization of particle multiplicity distributions in hadronic collisions. These techniques avoid model-dependent treatment of the underlying event activity, hadronization effects, and other effects that impact the description of particle activity between the jets that are embedded in Monte Carlo events.

\section{A. Background for jet-gap-jet events}

In the first approach, a separate $N_{\text {tracks }}$ distribution is obtained from a sample of events where the two leading jets are reconstructed on the same side of the CMS detector $\left(\eta^{\mathrm{jet} 1} \eta^{\mathrm{jet} 2}>0\right)$ with jets satisfying the requirements $1.4<$ $\left|\eta^{\text {jet }}\right|<4.7$ and $p_{\mathrm{T}}^{\text {jet }}>40 \mathrm{GeV}$. The independent sample of events where jets are produced on the same side is referred to as "SS dijet sample." The nominal sample, where jets are reconstructed on opposite sides of the detector $\left(\eta^{\text {jet1 }} \eta^{\text {jet2 }}<0\right)$, is denoted by "OS dijet sample." To suppress single-diffractive dijet contributions in the SS sample (dijet production with a forward pseudorapidity gap), which could affect the shape of the multiplicity distribution at very low multiplicities, at least one calorimeter tower with a minimum energy of $5 \mathrm{GeV}$ above the calorimeter noise level in the forward region opposite to the dijet system within $3<|\eta|<5.2$ is required. This SS method for estimating the color-exchange contributions in jetgap-jet analyses has been used by the CDF and CMS Collaborations [44-47].

Although the multiplicity distribution of charged particles in $|\eta|<1$ has a similar shape in the SS and the OS dijet samples, the SS dijet sample has a lower mean $N_{\text {tracks }}$ than the OS sample. To compensate for this difference and obtain a better superposition of the $N_{\text {tracks }}$ distributions of the SS and the OS dijet samples for multiplicities of $N_{\text {tracks }}>2$, the $\eta$ region for the SS dijet sample is adjusted. The adjustment is estimated by matching the mean multiplicity of the distributions of the SS and OS samples by varying the pseudorapidity gap width in the SS sample. The optimal $\eta$ interval for the SS dijet sample is $|\eta|<1.2$, consistent with findings by the CDF and CMS Collaborations at lower $\sqrt{s}$ [44-47]. The multiplicity distribution in the SS sample is then normalized to the one of the OS dijet sample in an interval dominated by 
color-exchange dijet events, $3 \leq N_{\text {tracks }} \leq 40$. The number of events of the SS sample in the first multiplicity bins $N_{\text {tracks }}<3$ becomes the estimated number of colorexchange events contributing in the color-singlet exchange signal region. This is illustrated in the left panel of Fig. 5 for one of the bins in the region $40<p_{\mathrm{T}}^{\text {jet2 }}<50 \mathrm{GeV}$ used in the analysis. An excess of OS dijet events at low multiplicities above the expected color-exchange events is observed, which is interpreted as the contribution of hard color-singlet exchange dijet events. The $f_{\text {CSE }}$ fraction is observed to stabilize up to $N_{\text {tracks }}=2$ with the SS method, within the statistical uncertainties, and thus this is the integration region used for the $f_{\mathrm{CSE}}$ extraction in the analysis. For events at low nonzero $N_{\text {tracks }}$, strong correlations in $\eta-\phi$ between the charged particles and the jets are observed. This suggests that events with low nonzero $N_{\text {tracks }}$ are due to charged particle constituents of the jet falling into the $|\eta|<1$ region.

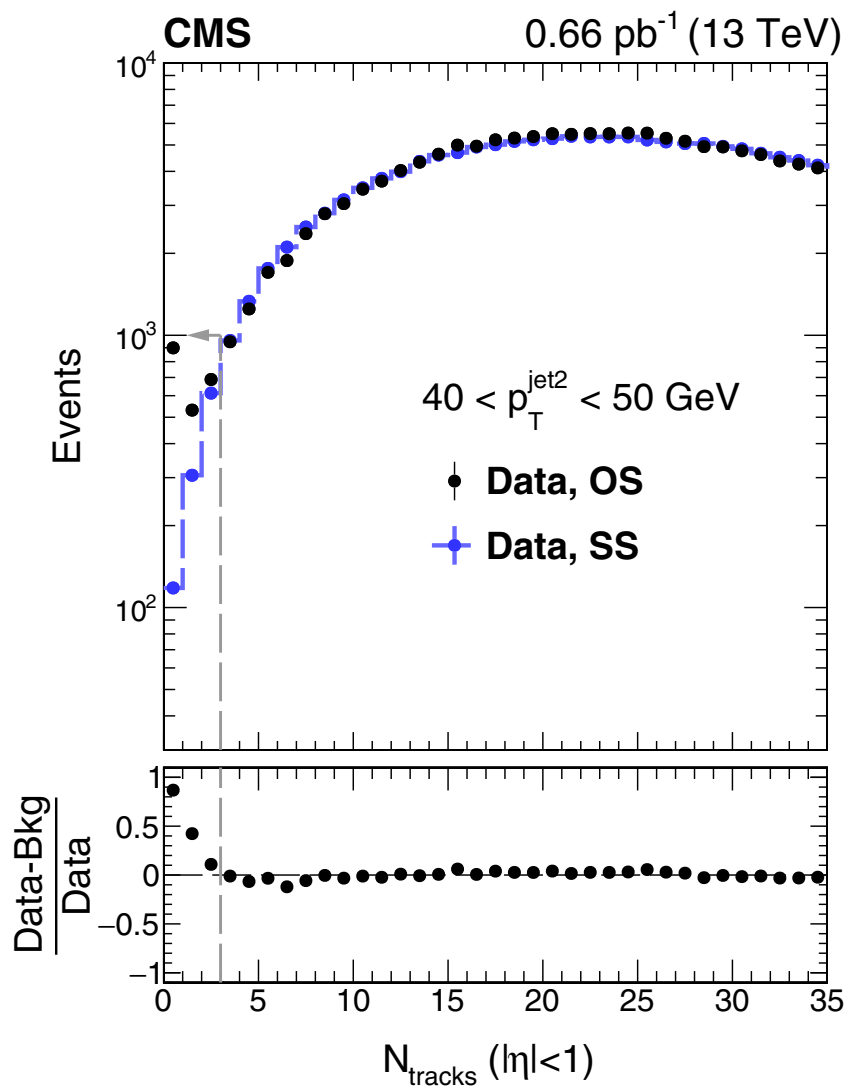

The second method used to estimate the color-exchange background relies on a fit to the $N_{\text {tracks }}$ distribution with a negative binomial distribution (NBD) function. This distribution is used to describe $N_{\text {tracks }}$ distributions with underlying color charge exchanges in hadronic collisions [80,81], as first reported by the UA5 Collaboration [82,83] at $\sqrt{s}=540 \mathrm{GeV}$. The NBD functional form has also been used to describe $p p$ collision data at several $\sqrt{s}$ values by the ALICE Collaboration [84]. The NBD function is less successful in describing the high multiplicity tails of $N_{\text {tracks }}$ distributions for $\sqrt{s}$ larger than $900 \mathrm{GeV}[83,84]$, and requires the use of more complex phenomenological parametrizations necessary for very wide multiplicity intervals. For the study of jet-gap-jet events, a single NBD function fit is sufficient, since the main focus is at low $N_{\text {tracks. }}$. The NBD method for estimating the color-exchange contributions in jet-gap-jet analyses has been used by the D0 and CMS Collaborations [41-43,47].

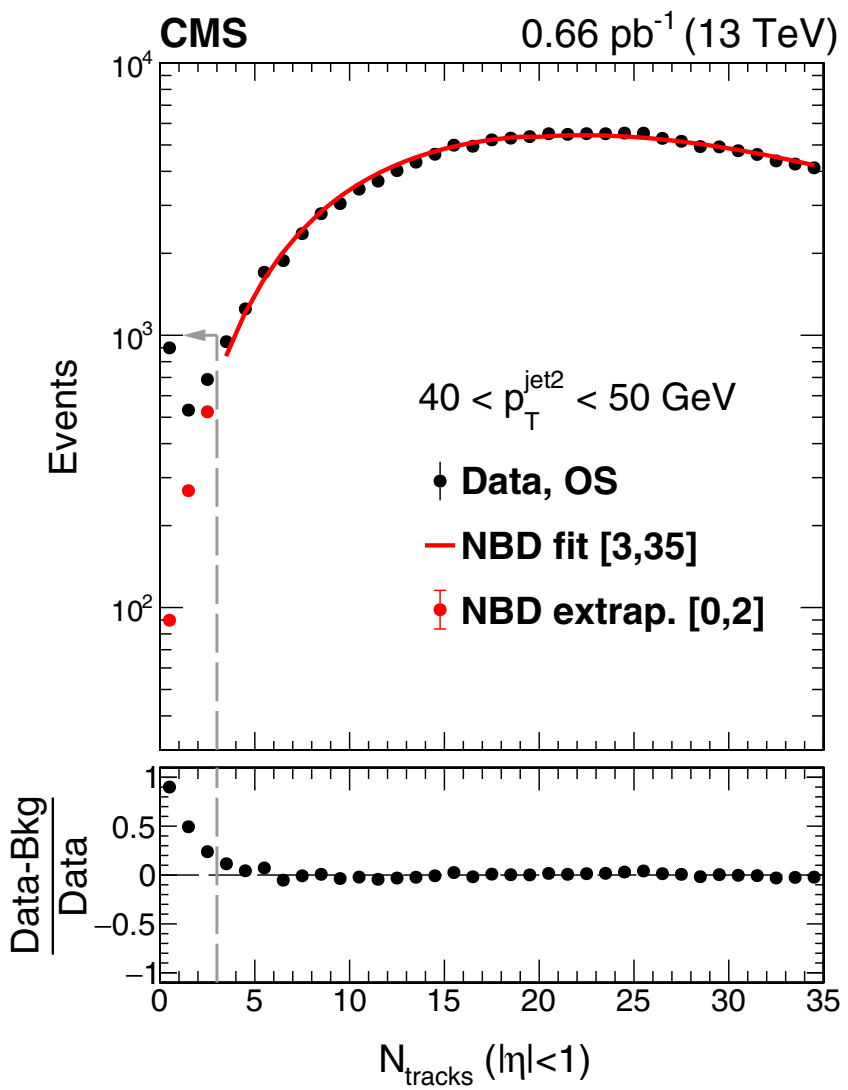

FIG. 5. Charged particle multiplicity distribution $N_{\text {tracks }}$ in the $|\eta|<1$ region for charged particle tracks with $p_{\mathrm{T}}>200 \mathrm{MeV}$ for opposite side (OS) dijet events satisfying $\eta^{\text {jet1 }} \eta^{\text {jet2 }}<0$ with $40<p_{\mathrm{T}}^{\text {jet2 }}<50 \mathrm{GeV}$. The vertical bars, which represent statistical uncertainties, are smaller than the markers for most data points. Results from color-exchange dijet background estimation based on the same side (SS) dijet events and the negative binomial distribution (NBD) function fit are shown on the left and right panels, respectively. The NBD function is fit in the interval $3 \leq N_{\text {tracks }} \leq 35$, and extrapolated to $N_{\text {tracks }}=0$. The dashed-line arrow represents the jet-gap-jet signal region used in the analysis, $N_{\text {tracks }} \leq 2$. The vertical bars of the NBD extrapolation points, which are smaller than the markers, represent the uncertainty in the extrapolation based on the fit parameter uncertainties extracted in the $3 \leq N_{\text {tracks }} \leq 35$ interval. The fraction $f_{\text {CSE }}$ corresponds to the ratio of the excess of events at low multiplicities relative to the integrated number of events, as described in the text. 
The NBD function is fit in the interval $3 \leq N_{\text {tracks }} \leq 35$, which is expected to be dominated by color-exchange dijet events. The range of $3 \leq N_{\text {tracks }} \leq 35$ also compares better to the $7 \mathrm{TeV}$ analysis, since the shape of the $N_{\text {tracks }}$ distribution is similar. The NBD function is extrapolated to $N_{\text {tracks }}=0$ to estimate the contribution of colorexchange dijet background counts. This is illustrated in the right panel of Fig. 5 for one of the bins, $40<p_{\mathrm{T}}^{\text {jet2 }}<50 \mathrm{GeV}$, used in the analysis. As with the SS method, an excess at low $N_{\text {tracks }}$ over the NBD extrapolation is observed. The fraction $f_{\mathrm{CSE}}$ is observed to stabilize by integrating the excess up to $N_{\text {tracks }}=2$ with the NBD method, within the statistical uncertainties, and hence this is the integration region used to extract $f_{\mathrm{CSE}}$. The estimated color-exchange dijet yield in the signal region is stable with respect to variations of the starting and ending points of the fit region, as verified explicitly by changing the fit interval to $3 \leq N_{\text {tracks }} \leq 25,3 \leq N_{\text {tracks }} \leq 45$, or $4 \leq N_{\text {tracks }} \leq 35$. The shape of the $N_{\text {tracks }}$ distribution is very similar for events with low $\Delta \eta_{\mathrm{jj}}$ (more central dijets) compared with those with large $\Delta \eta_{\mathrm{jj}}$ (very forward-backward dijet configurations). This is because, for the majority of the events, the gap region is far from the edges of the jets due to the $\left|\eta^{\mathrm{jet}}\right|>1.4$ requirement, which reduces the contamination of soft radiation from the jet.

The NBD method is used to extract the main results in the analysis, since it computes the fraction $f_{\mathrm{CSE}}$ as a function of the kinematic variables of interest. It also provides for a more direct comparison with the previous measurement by CMS at $\sqrt{s}=7 \mathrm{TeV}$ [47], where the main results are extracted with an NBD function fit in similar $N_{\text {tracks }}$ intervals. The SS method is used for systematic checks in the analysis. The SS method overestimates the contribution of color-exchange dijet events by about $15 \%$ relative to the results extracted with the NBD method in $40<p_{\mathrm{T}}^{\text {jet} 2}<50 \mathrm{GeV}$, and by about $1-5 \%$ for larger values of $p_{\mathrm{T}}^{\mathrm{jet} 2}>50 \mathrm{GeV}$. These differences are taken as a systematic uncertainty.

The performance of the NBD method is tested on the $N_{\text {tracks }}$ distribution of the SS dijet sample by performing the NBD fit in the range $3 \leq N_{\text {tracks }} \leq 35$. The extrapolation of the fit results to $N_{\text {tracks }}=0$ agrees with the SS data. As an additional check, a subset of the OS dijet sample characterized by the presence of a third leading jet with $p_{\mathrm{T}}{ }^{\text {jet3 }}>$ $15 \mathrm{GeV}$ and $\left|\eta^{\mathrm{jet} 3}\right|<1$ is analyzed. This selection yields a trijet sample enriched in color-exchange events. The NBD function fit describes correctly the $N_{\text {tracks }}$ distribution of this trijet sample, further confirming the validity of the NBD approach.

The $f_{\text {CSE }}$ fractions are extracted from the data using dijet yields uncorrected for detector effects. No unfolding of the data is necessary, since reconstruction, resolution, and migration effects cancel in the ratio of yields in $f_{\mathrm{CSE}}$. The number of color-singlet exchange dijet events in the numerator of Eq. (1) does not depend on track reconstruction inefficiencies; the latter only influence the color-exchange dijet events in the denominator of Eq. (1), which are subtracted in the analysis. Simulation events show that the results do not change within the statistical uncertainties if hadron-level or detector-level variables are used. This was also true for the $7 \mathrm{TeV}$ CMS paper [47].

For these simulation studies, inclusive dijet events (with no hard color-singlet exchange contributions) were simulated using the leading order (LO) PYTHIA8 Monte Carlo event generator [85] (version 8.212) with the PDF set NNPDF2.3LO [86,87]. The PYTHIA8 generator relies on a parton showering algorithm for resummation of soft and collinear gluon emissions at leading-logarithm accuracy, and on the Lund string fragmentation model for hadronization effects [88]. The underlying event tune CUETP8M1 [89] is used, together with initial- and final-state radiation effects. Hard color-singlet exchange events are simulated with the HERwIG6 Monte Carlo event generator [90] (version 6.520) with the PDF set CTEQ6L1 [91]. The HERWIG6 generator simulates events with hard color-singlet exchange between two partons following predictions based on simplified leading-logarithm BFKL calculations. Hadronization effects in HERWIG6 are based on the cluster fragmentation model [92]. The JIMMY package [93] is used to supplement MPI. A detailed simulation of the CMS detector response is performed with the GEANT4 toolkit [94]. The reconstruction of these simulated events uses the same algorithms as the data. Stable particles, whose decay length is greater than $20 \mathrm{~mm}$, are used for jet reconstruction and measurement of the charged particle multiplicity distribution between the jets in these studies. The hadron-level results on $f_{\mathrm{CSE}}$ are compared with those obtained when considering the detector response, and agree within the statistical uncertainties, provided that the signal extraction is performed at most at $N_{\text {tracks }}<3$. The $f_{\mathrm{CSE}}$ values in simulation are matched to those in data for these studies. For a check of the background subtraction methods used in the analysis, the $f_{\text {CSE }}$ values calculated with PYTHIA8 (color-exchange dijet events, no jet-gap-jet signal) are compared, and found consistent with those extracted using the SS or NBD methods, within the statistical uncertainties.

\section{B. Background for proton-gap-jet-gap-jet events}

In the sample with intact protons, the contribution of protons from pileup interactions and beam halo activity must be subtracted. The residual contamination that survives the selection requirement $\xi_{p}(\mathrm{PF})-\xi_{p}(\mathrm{RP})<0$, as noted in Sec. IV B, is estimated using an event mixing procedure that mimics the beam background contribution in the nominal sample, as described below.

Events from the inclusive dijet sample are paired with uncorrelated protons from events in the zero-bias data sample. The dijet events should satisfy the same event 
selection requirement described in Sec. IV. The number of events from this event mixing procedure is normalized to data with $\xi_{p}(\mathrm{PF})-\xi_{p}(\mathrm{RP})>0$, which is dominated by beam background events. Then, the number of events with $\xi_{p}(\mathrm{PF})-\xi_{p}(\mathrm{RP})<0$ is the estimated number of beam background events present in the nominal sample. The results of this procedure are presented in Fig. 6. Beam background contamination constitutes 18.6 and $21.5 \%$ of the sample in sectors 45 and 56, respectively. Similar procedures have been used in Refs. [75,95-100]. The distribution of $N_{\text {tracks }}$ from beam background, shown in Fig. 7 , is determined from the event mixing procedure. A larger number of events in the $\xi_{p}(\mathrm{PF})-\xi_{p}(\mathrm{RP})>0$ region is observed for intact protons detected in sector 56 . This is indicative of a larger beam background contamination in sector 56 in comparison to sector 45. A higher beam background activity in sector 56 has been previously observed by CMS and TOTEM in the $8 \mathrm{TeV}$ singlediffractive dijet measurement [75].

Standard single-diffractive dijet events can yield a central gap between the jets by fluctuations in $N_{\text {tracks }}$, analogous to the fluctuations of color-exchange dijet events in inclusive dijet production. The methods introduced in Sec. VII A are used to estimate these contributions with modifications that account for differences in the sample with intact protons. Generally, the $N_{\text {tracks }}$ is lower in events with an intact proton than in inclusive dijet production events. For events passing the dijet and forward proton selection requirements, the mean multiplicity in the $|\eta|<1$ region is $\left\langle N_{\text {tracks }}\right\rangle \approx 17$, compared to the larger $\left\langle N_{\text {tracks }}\right\rangle \approx 28$ in inclusive dijet production. This is consistent with the overall suppression of spectator parton interactions and lower energy available for production of particles in singlediffractive events. Since the $N_{\text {tracks }}$ distributions in sectors 45 and 56 are similar in shape, the $N_{\text {tracks }}$ values from the two sectors are summed for the analysis.

The first approach is the SS method. For the analysis with intact protons, the definition of the SS dijet sample introduced in Sec. VII A cannot be used. The mean $\eta$ of the jets is not centered at zero in single-diffractive events. This is because single-diffractive dijet events are intrinsically boosted along the beam direction, in a direction opposite to the scattered proton. Thus, in considering single-diffractive dijet events located in the same hemisphere of the CMS detector, the $N_{\text {tracks }}$ in the $|\eta|<1.2$ region is directly influenced by the intrinsic boost effects. To account for this, the $N_{\text {tracks }}$ distribution of the SS dijet sample is instead measured in intervals of $-2.0<\eta<0.4$ or $-0.4<\eta<2.0$ for protons detected in sector 45 or 56, respectively. These intervals are determined based on the mean jet $\eta$ in the data for events with an intact proton in sectors 45 and 56, which corresponds to boosts of about 0.8 units in negative and positive $\eta$, respectively. The two leading jets are located on the same side relative to these $\eta$ intervals, i.e., $\eta^{\text {jet }}<-2.2$ or $\eta^{\text {jet }}>0.6$ for intact protons in sector 45 and $\eta^{\text {jet }}<-0.6$ or
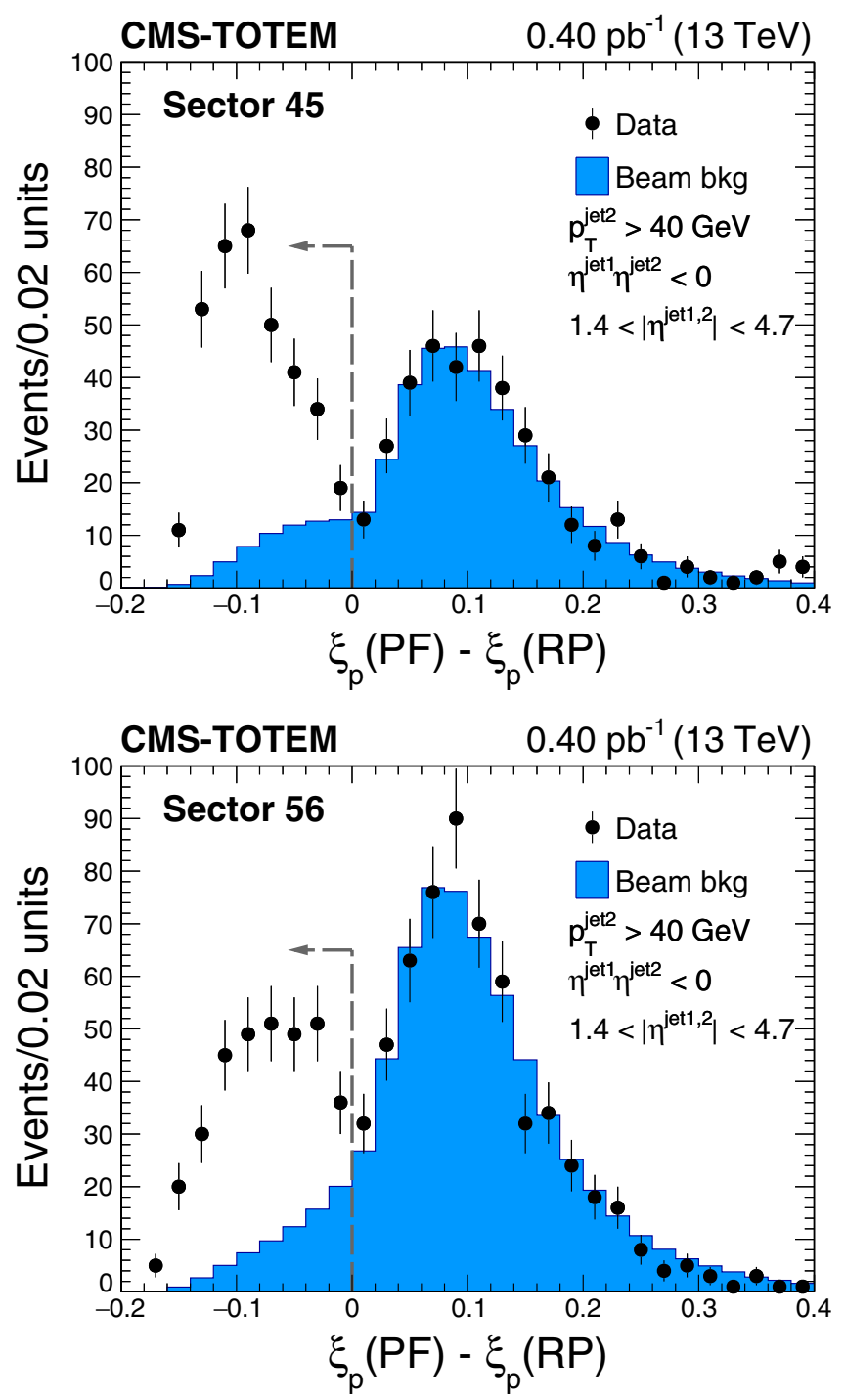

FIG. 6. Distribution of $\xi_{p}(\mathrm{PF})-\xi_{p}(\mathrm{RP})$ in sectors 45 (Upper) and 56 (Lower) in data, where $\xi_{p}(\mathrm{PF})$ and $\xi_{p}(\mathrm{RP})$ denote the fractional momentum loss of the proton reconstructed with the particle-flow (PF) candidates of CMS and the Roman pots (RP) of TOTEM, respectively. The vertical bars indicate statistical uncertainties only. The estimated background contamination (beam background events) is represented by the filled histogram, and is estimated from the data, as described in the text. The statistical uncertainties of the beam background histograms are smaller than the histogram lines. No central gap is required for this plot. The dashed-line arrow represents the requirement applied in the analysis to remove most of the beam background contribution.

$\eta^{\text {jet }}>2.2$ for protons in sector 56 . The location of the jet axes is 0.2 units away from the $\eta$ interval, as in the construction of the SS dijet sample of Sec. VII A. The resulting $N_{\text {tracks }}$ distribution of the SS dijet sample matches that of the OS sample at moderate multiplicities after these adjustments. The $N_{\text {tracks }}$ distribution of the SS dijet sample is normalized to that of the nominal sample in the range $2 \leq N_{\text {tracks }} \leq 40$. The number of events of the SS dijet 

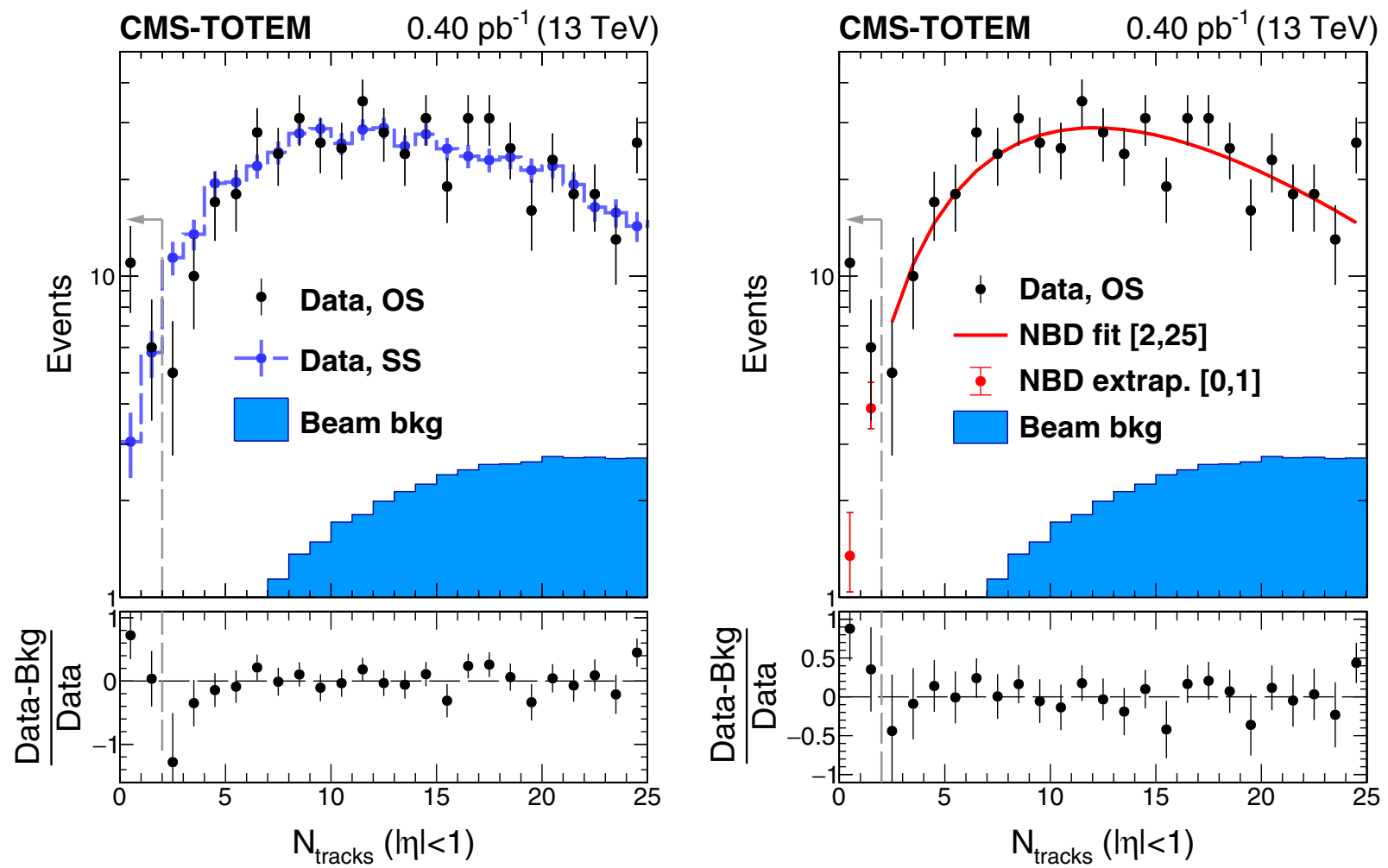

FIG. 7. Charged particle multiplicity distribution in the $|\eta|<1$ region after the dijet and proton selection. Opposite side (OS) dijet events satisfy $\eta^{\text {jet } 1} \eta^{\text {jet} 2}<0$. The vertical bars represent the statistical uncertainties. The filled histogram represents the residual beam background contamination. The contribution of standard diffractive dijet events that feature a central gap is modeled with the same side (SS) dijet events (left) and with the negative binomial distribution (NBD) function fit (right), as described in the text. The NBD function is fit in the interval $2 \leq N_{\text {tracks }} \leq 25$, and extrapolated to $N_{\text {tracks }}=0$. The dashed-line arrow represents the region $N_{\text {tracks }}<2$ used for signal extraction in the analysis. The vertical bars of the NBD extrapolation points represent the uncertainty in the extrapolation based on the fit parameter uncertainties extracted in the $2 \leq N_{\text {tracks }} \leq 25$ interval.

sample in the lowest multiplicity bins is then used to estimate the standard single-diffractive dijet production at low multiplicities $N_{\text {tracks }} \leq 1$, as shown in Fig. 7. An excess of events over the expected background counts is observed, which is attributed to the presence of proton-gap-jet-gap-jet events.

The second approach is based on the NBD method introduced in Sec. VII A. The NBD function is fit in the interval $2 \leq N_{\text {tracks }} \leq 25$, and is then extrapolated to $N_{\text {tracks }}=0$ to estimate the contribution of standard diffractive dijet events that feature a central gap, as seen in Fig. 7. The upper bound at $N_{\text {tracks }}=25$ is chosen to include the lower mean $N_{\text {tracks }}$ of the dijet sample with intact protons, and, at the same time, to avoid the contribution by beam background contamination that dominates at high multiplicities. The NBD is fit before beam background subtraction. The result is the same if the fit is carried out after the beam background subtraction, which has an effect on the extracted $f_{\mathrm{CSE}}$ of less than $2 \%$. An excess over the NBD extrapolation results is observed in the data, which provides for an interpretation in terms of proton-gap-jetgap-jet events. The NBD method is used to extract the main results in the analysis, which facilitates a comparison with the jet-gap-jet results extracted in inclusive dijet production. Because of the lower mean value of $N_{\text {tracks }}$ and the smaller width of the $N_{\text {tracks }}$ distribution, the NBD fit extrapolation is more sensitive in jet-gap-jet events with an intact proton than in inclusive dijet events. This is quantified as part of the systematic uncertainties in the $f_{\mathrm{CSE}}$ extraction.

\section{SYSTEMATIC UNCERTAINTIES}

\section{A. Systematic uncertainties in the study of jet-gap-jet events}

The sources of systematic uncertainties for the $f_{\text {CSE }}$ fraction measurement are

Jet energy scale: The $p_{\mathrm{T}}$ of each jet is varied with $p_{\mathrm{T}} \rightarrow p_{\mathrm{T}} \pm \delta p_{\mathrm{T}}\left(p_{\mathrm{T}}, \eta\right)$, where $\delta p_{\mathrm{T}}\left(p_{\mathrm{T}}, \eta\right)$ is the jet energy scale uncertainty as a function of the jet $p_{\mathrm{T}}$ and $\eta$. The new jet collection is reordered in $p_{\mathrm{T}}$, and the analysis is repeated. The difference in the extracted fraction $f_{\text {CSE }}$ relative to the results found with the nominal jet energy corrections is a measure of the associated systematic uncertainty. The resulting relative uncertainty is $0.5 \%-6.0 \%$. 
Track quality: The selection criteria used to define highpurity tracks are loosened and the difference in $f_{\mathrm{CSE}}$ with respect to the nominal selection is taken as the associated systematic uncertainty. The loose quality criteria correspond to the minimum requirements yielding well-reconstructed tracks in the CMS detector, as described in Ref. [66]. The corresponding uncertainty in $f_{\mathrm{CSE}}$ is $1.5 \%-8.0 \%$.

Charged particle $p_{\mathrm{T}}$ threshold: Charged particles with $p_{\mathrm{T}}<200 \mathrm{MeV}$ are not considered in identifying a central gap. To study the sensitivity of the results to this threshold, the analysis is repeated with $p_{\mathrm{T}}$ thresholds of 150 and $250 \mathrm{MeV}$ for particles with $|\eta|<1$. The corresponding relative differences in the measured $f_{\mathrm{CSE}}$ fractions are $1.1 \%-5.8 \%$ and are assigned as systematic uncertainties.

Background subtraction method: The background determined using the SS method is compared with the adopted NBD background approach, and the difference is the associated systematic uncertainty. This reflects the imperfect knowledge of the $N_{\text {tracks }}$ distributions for color-exchange dijet events. At lower $p_{\mathrm{T}}^{\mathrm{jet} 2}$ values, with $40<p_{\mathrm{T}}^{\text {jet2 }}<50 \mathrm{GeV}$, the relative systematic uncertainty is $14.6 \%$, whereas for larger values, $p_{\mathrm{T}}^{\text {jet2 }}>80 \mathrm{GeV}$, it is $2 \%-5 \%$.

NBD fit parameters: The NBD function has three free parameters, including an overall normalization. The colorexchange dijet yields in the signal region are recalculated by varying the NBD fit parameters within their uncertainties. Correlations between the fit parameters are included in this procedure. The maximal differences relative to the nominal results are a measure of the associated systematic uncertainty. These calculations result in a relative uncertainty of less than $2.6 \%$ in the extracted $f_{\text {CSE }}$.

Functional form of the fit: To quantify the systematic uncertainty associated with the functional form chosen to fit the $N_{\text {tracks }}$ distribution at large multiplicities, the $N_{\text {tracks }}$ distribution is fit instead with a double NBD function (a sum of two NBD functions) to extract $f_{\text {CSE }}$. The double NBD function has been found to be an alternative empirical parametrization of charged particle multiplicities in hadronic collisions at various $\sqrt{s}$, particularly for very wide $N_{\text {tracks }}$ intervals $[81,83,84]$. The symmetrized difference of the $f_{\text {CSE }}$ extracted with the double NBD fit with respect to the nominal $f_{\text {CSE }}$ fraction is taken as the respective systematic uncertainty. The relative uncertainty in the extracted $f_{\mathrm{CSE}}$ is $2 \%-7 \%$.

A summary of the systematic uncertainties is presented in Table I. The systematic uncertainties are added in quadrature and the total bin-specific systematic uncertainty varies between $7 \%$ and $23 \%$.

As mentioned in Sec. V, no neutral particles are used in the definition of the pseudorapidity gap because of the relatively large $p_{\mathrm{T}}$ thresholds above the calorimeter noise for neutral hadrons and photons. Most dijet events with low $N_{\text {tracks }}$ in the region $|\eta|<1$ have little, if any, neutral particle activity in that region. Simulation studies that include the detector response, based on the samples described in Sec. VII A, suggest that the neutral hadron and photon activity observed in data originate from the emission of jet constituents into the $|\eta|<1$ region, together with residual contributions of the calorimeter noise. The $f_{\text {CSE }}$ fractions remain mostly unaffected if the contribution of neutral particles at central $\eta$ is included in the analysis. In particular, if the vector $p_{\mathrm{T}}$ sum of the neutral hadrons and photons for $|\eta|<1$ is required to be less than $15 \mathrm{GeV}$, the results for $f_{\mathrm{CSE}}$ are the same, within the statistical uncertainties of $f_{\mathrm{CSE}}$. This is consistent since the color-exchange dijet background is already subtracted in the determination of $f_{\mathrm{CSE}}$.

TABLE I. Relative systematic uncertainties in percentage for the measurements of $f_{\text {CSE }}$ in jet-gap-jet and proton-gap-jet-gap-jet events. The jet-gap-jet results summarize the systematic uncertainties in bins of the kinematic variables of interest $p_{\mathrm{T}}^{\mathrm{jet} 2}, \Delta \eta_{\mathrm{jj}}$, and $\Delta \phi_{\mathrm{jj}}$. When an uncertainty range is given, the range of values is representative of the variation found in $f_{\mathrm{CSE}}$ in bins of the kinematic variables of interest.

\begin{tabular}{|c|c|c|c|c|}
\hline \multirow[b]{2}{*}{ Source } & \multicolumn{3}{|c|}{ Jet-gap-jet $(\%)$} & \multirow[b]{2}{*}{ Proton-gap-jet-gap-jet $(\%)$} \\
\hline & $\Delta \eta_{\mathrm{jj}}$ & $p_{\mathrm{T}}^{\mathrm{jet} 2}$ & $\Delta \phi_{\mathrm{jj}}$ & \\
\hline Jet energy scale & $1.0-5.0$ & $1.5-6.0$ & $0.5-3.0$ & 0.7 \\
\hline Track quality & $6.0-8.0$ & $5.4-8.0$ & $1.5-8.0$ & 8 \\
\hline Charged particle $p_{\mathrm{T}}$ threshold & $2.0-5.8$ & $1.6-4.0$ & $1.1-5.8$ & 11 \\
\hline Background subtraction method & $4.7-15$ & $2-15$ & 12 & 28 \\
\hline NBD fit parameters & $0.8-2.6$ & $0.6-1.7$ & $0.1-0.6$ & 7.0 \\
\hline Functional form of the fit & $2-7.3$ & $1.4-8.0$ & $0.6-7.8$ & 11.5 \\
\hline NBD fit interval & $\cdots$ & $\ldots$ & $\ldots$ & 12 \\
\hline Calorimeter energy scale & $\cdots$ & $\cdots$ & $\cdots$ & 5.0 \\
\hline Horizontal dispersion & $\cdots$ & $\cdots$ & $\cdots$ & 6.0 \\
\hline Fiducial selection requirements & $\cdots$ & $\cdots$ & $\cdots$ & 2.6 \\
\hline Total & $7-23$ & $9-15$ & $12-18.5$ & 35 \\
\hline
\end{tabular}




\section{B. Systematic uncertainties in the study of proton-gap-jet-gap-jet events}

In addition to the sources of systematic uncertainties described in Sec. VIII A, the following sources of systematic uncertainties that affect the extraction of $f_{\mathrm{CSE}}$ in proton-gap-jet-gap-jet events are considered:

NBD fit interval: Because of the lower mean $N_{\text {tracks }}$ and the limited sample size, the NBD fit extrapolation is more sensitive to the fit interval in events with an intact proton than in inclusive dijet production. The color-exchange dijet background for intervals of $2 \leq N_{\text {tracks }} \leq 15$ and $2 \leq$ $N_{\text {tracks }} \leq 35$ is evaluated. The difference of the extracted $f_{\mathrm{CSE}}$ value for these intervals relative to that for the nominal interval $2 \leq N_{\text {tracks }} \leq 25$ is taken as the associated systematic uncertainty. Based on these studies an uncertainty of $12 \%$ is assigned to the extracted $f_{\mathrm{CSE}}$. The difference of the measured $f_{\text {CSE }}$ value using the fit interval $3 \leq N_{\text {tracks }} \leq 25$ relative to the nominal fit interval is negligible.

Calorimeter energy scale: Beam background contributions are suppressed via the requirement $\xi_{p}(\mathrm{PF})-\xi_{p}(\mathrm{RP})<0$ in the analysis. Since $\xi_{p}(\mathrm{PF})$ is constructed from the PF candidates of the CMS experiment, it is affected by the energy calibration uncertainties of each PF candidate. The impact on $\xi_{p}(\mathrm{PF})$ is estimated by varying the energy of the PF candidates conservatively by $\pm 10 \%$ [67]. The corresponding relative difference in the extracted $f_{\mathrm{CSE}}$ value is $5 \%$, and is included as the associated systematic uncertainty.

Horizontal dispersion: The determination of $\xi_{p}(\mathrm{RP})$ depends on the LHC optics parametrization in the transport matrix, which connects the kinematics of the proton at the interaction point with those measured at the RPs. The horizontal dispersion term in the transport matrix directly affects the measurement of $\xi_{p}(\mathrm{RP})$ [64]. The associated systematic uncertainty is estimated by conservatively scaling the value of $\xi_{p}(\mathrm{RP})$ by $\pm 10 \%$, and repeating the analysis. The $f_{\text {CSE }}$ has an uncertainty of $6 \%$.

Fiducial selection requirements for $x(\mathrm{RP})-y(\mathrm{RP})$ coordinates at the RPs: The vertical and horizontal fiducial requirements are varied by 0.2 and $1 \mathrm{~mm}$, respectively. The relative difference of the $f_{\mathrm{CSE}}$ result with respect to that obtained with the nominal fiducial $x(\mathrm{RP})-y(\mathrm{RP})$ requirements is less than $2.6 \%$, and is assigned as the corresponding systematic uncertainty.

The systematic uncertainties are summarized in Table I. The systematic uncertainties related to the jet reconstruction and central gap definition are larger in the proton-gap-jet-gap-jet study. The total systematic uncertainty is calculated as the quadratic sum of the individual contributions, and it has a value of $35 \%$.

\section{RESULTS}

\section{A. Results for jet-gap-jet events in inclusive dijet production}

The measured fractions $f_{\mathrm{CSE}}$ are presented in Fig. 8 and Tables II-IV. As a function of $\Delta \eta_{\mathrm{jj}}$, the $f_{\mathrm{CSE}}$ fraction shows a uniform increase from 0.4 to $1.0 \%$ for $\Delta \eta_{\mathrm{jj}}$ between 3 and 6 units. Within the experimental uncertainties, $f_{\mathrm{CSE}}$ is about $0.7 \%$, and shows little, if any, dependence on $p_{\mathrm{T}}^{\mathrm{jet} 2}$. As a function of $\Delta \phi_{\mathrm{jj}}$ between the two leading jets, the $f_{\mathrm{CSE}}$ fraction exhibits a peak near $\Delta \phi_{\mathrm{jj}}=\pi$ with a value of $1 \%$, which suggests that jet-gap-jet events are more strongly correlated in the transverse plane than inclusive dijet events. A constant value of about $0.4 \%$ is found for $\Delta \phi_{\mathrm{jj}}<2.8$; this implies that color-singlet exchange dijet events decorrelate at a similar rate as color-exchange dijet events in this interval.

The present results are compared with BFKL-based theoretical calculations of Royon, Marquet, Kepka (RMK) [54,55] and Ekstedt, Enberg, Ingelman, Motyka (EEIM) $[53,56]$, the results of which are shown in Fig. 8. The RMK and EEIM model predictions include dominant NLL corrections to the BFKL evolution of the parton-level cross section using LO impact factors. The RMK predictions are supplemented with a gap survival probability of $|\mathcal{S}|^{2}=10 \%$, whose value is used to match the $f_{\mathrm{CSE}}$ values observed in data. The RMK predictions use an updated parametrization of the BFKL NLL amplitudes that include the larger phase space available at LHC energies [101], which are then implemented in the HERWIG6 generator [55]. The theoretical uncertainties in the RMK prediction are due to renormalization and factorization scale uncertainties in the BFKL calculation [55]. The EEIM predictions include soft rescattering effects based on MPI or MPI supplemented with SCI. In the EEIM approach, the spectator parton activity originating from perturbative gluons is modeled in terms of initial- and final-state parton showers, MPI, and hadronization processes, as implemented in PYTHIA6 [53,56]. The SCI accounts for possible gap destruction effects caused by color exchange with negligible momentum transfer that rearrange the color field created in the $p p$ collision [53]. The free parameters of the SCI model are fit to describe the previous $7 \mathrm{TeV}$ measurement by CMS [56]. The remaining nonperturbative corrections are either modeled with a survival probability of $|\mathcal{S}|^{2}=1.2 \%$ to match the $f_{\mathrm{CSE}}$ value found in data (purple dashed line in Fig. 8) or with SCI (orange dotted line in Fig. 8). The theoretical uncertainties in the EEIM model predictions are dominated by the cutoff $p_{\mathrm{T}}$ scale used for MPI in the simulation.

According to both the RMK and EEIM model calculations, $f_{\mathrm{CSE}}$ should have a weak dependence on $p_{\mathrm{T}}^{\mathrm{jet} 2}$. Within the uncertainties, this feature is consistent with the observed $f_{\text {CSE }}$ values. The predictions by RMK and EEIM (with MPI only) yield a decreasing $f_{\mathrm{CSE}}$ with increasing $\Delta \eta_{\mathrm{jj}}$. This is in disagreement with the data, which show a $f_{\mathrm{CSE}}$ that generally grows with larger $\Delta \eta_{\mathrm{jj}}$. The EEIM model predictions, when supplemented with SCI, correctly describe $f_{\mathrm{CSE}}$ as a function of $\Delta \eta_{\mathrm{jj}}$ within the uncertainties. The predictions of the RMK model for $f_{\mathrm{CSE}}$ as a function of $\Delta \phi_{\mathrm{jj}}$ are consistent with the 

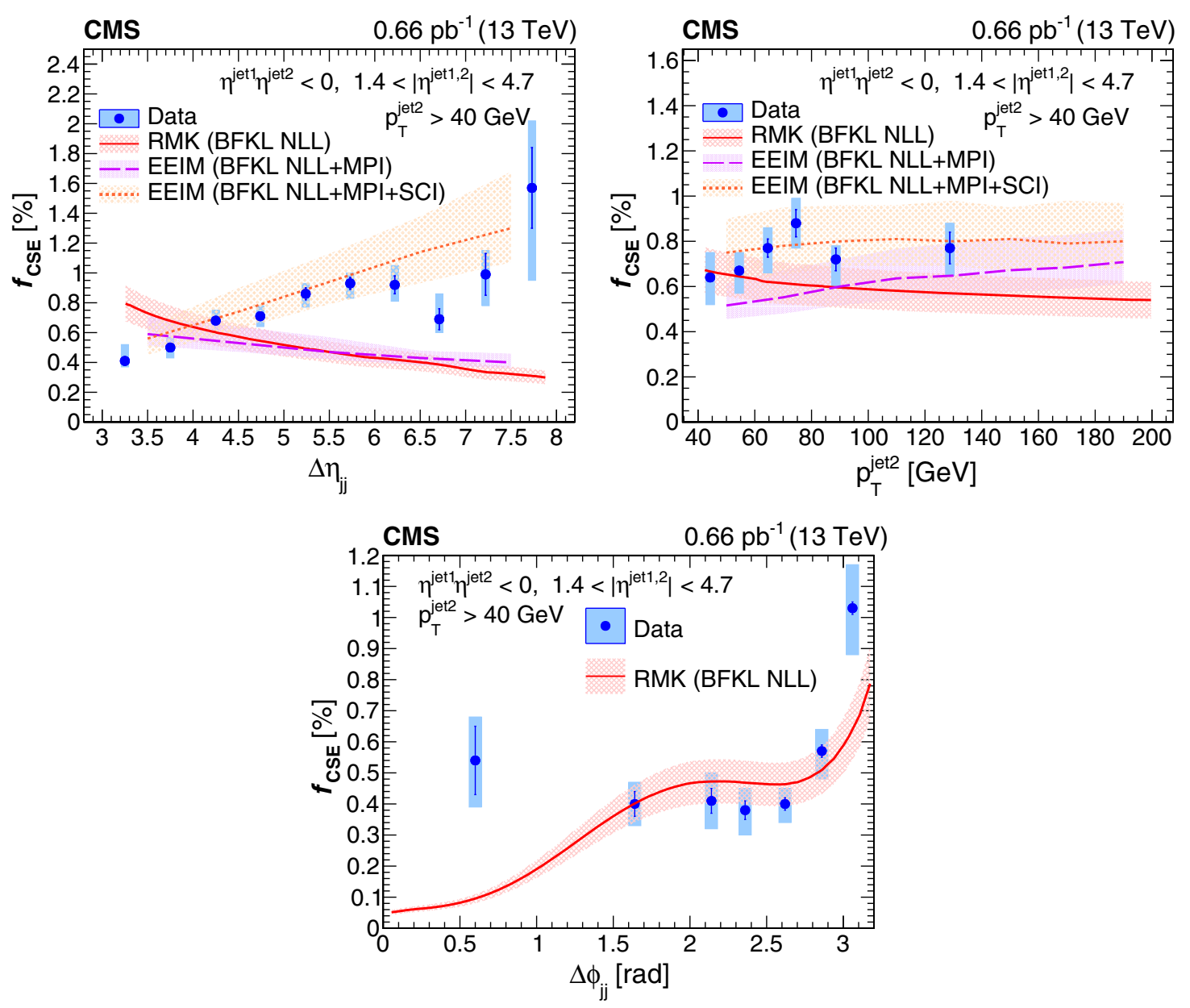

FIG. 8. Fraction of color-singlet exchange dijet events, $f_{\mathrm{CSE}}$, measured as a function of $\Delta \eta_{\mathrm{jj}}, p_{\mathrm{T}}^{\mathrm{jet} 2}$, and $\Delta \phi_{\mathrm{jj}}$ in $p p$ collisions at $\sqrt{s}=13 \mathrm{TeV}$. The vertical bars represent statistical uncertainties, while boxes represent the combination of statistical and systematic uncertainties in quadrature. The results are plotted at the mean values of $\Delta \eta_{\mathrm{jj}}$, $p_{\mathrm{T}}^{\mathrm{jet} 2}$, and $\Delta \phi_{\mathrm{jj}}$ in the bin. For a given plot of $f_{\mathrm{CSE}}$ versus a kinematic variable of interest $\left(p_{\mathrm{T}}^{\mathrm{jet} 2}, \Delta \eta_{\mathrm{jj}}\right.$, or $\left.\Delta \phi_{\mathrm{jj}}\right)$, the other kinematic variables are integrated over their allowed range. The red solid curve corresponds to theoretical predictions based on the RMK model [54,55] with gap survival probability of $|\mathcal{S}|^{2}=10 \%$. The EEIM model $[53,56]$ predictions with MPI-only contributions and $|\mathcal{S}|^{2}=1.2 \%$ or MPI + SCI are represented by the purple dashed and orange dotted curves, respectively. The bands around the curves represent the associated theoretical uncertainties. The EEIM model has only small contributions far from back-to-back jets since no hard NLO $2 \rightarrow 3$ processes are included, and thus predictions are not shown for the lower panel of $f_{\mathrm{CSE}}$ versus $\Delta \phi_{\mathrm{jj}}$.

data within the uncertainties for medium angular separations $1<\Delta \phi_{\mathrm{jj}}<3$, but underestimate the experimental result by about $10 \%$ near $\Delta \phi_{\mathrm{jj}}=\pi$. The model significantly underestimates the observed $f_{\mathrm{CSE}}$ for small angular separations with $\Delta \phi_{\mathrm{jj}}<1$. The EEIM model uses LO $2 \rightarrow 2$ hard processes resulting in back-to-back hard jets, such that $\Delta \phi_{\mathrm{jj}} \approx \pi$, with only small deviations due to the leading logarithmic parton showers, but no hard next-to-LO (NLO) $2 \rightarrow 3$ processes causing larger deviations.

Present calculations include partial corrections at NLO in $\alpha_{\mathrm{S}}$ within the BFKL framework, namely resummation of large logarithms of energy at NLL accuracy using LO impact factors. Higher-order corrections to impact factors are known to have significant effects in the description of similar processes, such as Mueller-Navelet jets [102]. Recently, major progress has been made in the calculation of NLO impact factors for the jet-gap-jet process [78,79]. These corrections have yet to be included in the BFKL theoretical calculations to complete the NLO analysis of the jet-gap-jet process.

In Fig. 9, the current results are compared with previous measurements of $f_{\mathrm{CSE}}$ with a central gap in $|\eta|<1$ by the D0 and CDF Collaborations at the Tevatron in $p p$ collisions at $\sqrt{s}=0.63$ and $1.8 \mathrm{TeV}[42,43,45,46]$, and by the CMS Collaboration in $p p$ collisions at $7 \mathrm{TeV}$ [47]. There are differences in the phase space volumes populated by the two leading jets, jet clustering algorithms, and distance parameters, which are described in the next 
TABLE II. Measured values of the fraction of color-singlet exchange events $f_{\mathrm{CSE}}$ in bins of the pseudorapidity difference between the two leading jets $\Delta \eta_{\mathrm{jj}}$. The first column indicates the $\Delta \eta_{\mathrm{jj}}$ intervals and the last column represents the measured fraction. The first and second uncertainties correspond to the statistical and systematic components, respectively. The results are integrated over the allowed $p_{\mathrm{T}}^{\mathrm{jet} 2}$ and $\Delta \phi_{\mathrm{jj}}$ values. The mean values of $\Delta \eta_{\mathrm{jj}}$ in the bin are given in the middle column.

\begin{tabular}{lcc}
\hline \hline$\Delta \eta_{\mathrm{jj}}$ & $\left\langle\Delta \eta_{\mathrm{jj}}\right\rangle$ & $f_{\mathrm{CSE}}[\%]$ \\
\hline $3.0-3.5$ & 3.24 & $0.41 \pm 0.02_{-0.04}^{+0.011}$ \\
$3.5-4.0$ & 3.75 & $0.50 \pm 0.02_{-0.07}^{+0.07}$ \\
$4.0-4.5$ & 4.25 & $0.68 \pm 0.02_{-0.06}^{+0.07}$ \\
$4.5-5.0$ & 4.74 & $0.71 \pm 0.03_{-0.06}^{+0.06}$ \\
$5.0-5.5$ & 5.24 & $0.86 \pm 0.04_{-0.08}^{+0.06}$ \\
$5.5-6.0$ & 5.73 & $0.93 \pm 0.04_{-0.09}^{+0.06}$ \\
$6.0-6.5$ & 6.22 & $0.92 \pm 0.06_{-0.09}^{+0.11}$ \\
$6.5-7.0$ & 6.71 & $0.69 \pm 0.07_{-0.05}^{+0.15}$ \\
$7.0-7.5$ & 7.22 & $0.99 \pm 0.14_{-0.07}^{+0.07}$ \\
$7.5-8.0$ & 7.73 & $1.57 \pm 0.27_{-0.56}^{+0.35}$ \\
\hline \hline
\end{tabular}

TABLE III. Measured values of the fraction of color-singlet exchange events $f_{\mathrm{CSE}}$ in bins of the subleading jet transverse momentum $p_{\mathrm{T}}^{\mathrm{jet} 2}$. The first column indicates the $p_{\mathrm{T}}^{\mathrm{jet} 2}$ bin intervals and the last column represents the measured fraction. The first and second uncertainties correspond to the statistical and systematic components, respectively. The results are integrated over the allowed $\Delta \eta_{\mathrm{jj}}$ and $\Delta \phi_{\mathrm{jj}}$ values. The mean values of $p_{\mathrm{T}}^{\mathrm{jet} 2}$ in the bin are given in the middle column.

\begin{tabular}{lcc}
\hline \hline$p_{\mathrm{T}}^{\mathrm{jet} 2}[\mathrm{GeV}]$ & $\left\langle p_{\mathrm{T}}^{\mathrm{je} 2}\right\rangle[\mathrm{GeV}]$ & $f_{\mathrm{CSE}}[\%]$ \\
\hline $40-50$ & 44.3 & $0.64 \pm 0.01_{-0.12}^{+0.11}$ \\
$50-60$ & 54.5 & $0.67 \pm 0.02_{-0.08}^{+0.10}$ \\
$60-70$ & 64.6 & $0.77 \pm 0.04_{-0.08}^{+0.08}$ \\
$70-80$ & 74.5 & $0.88 \pm 0.06_{-0.09}^{+0.09}$ \\
$80-100$ & 88.6 & $0.72 \pm 0.05_{-0.11}^{+0.04}$ \\
$100-200$ & 128.8 & $0.77 \pm 0.07_{-0.10}^{+0.09}$ \\
\hline \hline
\end{tabular}

paragraphs. Simulation studies that rely on hadron-level particle distributions, based on the samples described in Section VII A, indicate that the choice of the jet reconstruction algorithm (cone or anti- $k_{\mathrm{T}}$ algorithms) has a negligible effect on the shape of the charged particle multiplicity distribution between the jets. The value of the distance parameter $R$ influences the charged particle multiplicity distribution shape of jet-gap-jet signal events. For large values of $R$, it is less likely for charged particle constituents of the jet to populate the central $|\eta|<1$ region since the jet axes are further away from the edges of the gap region. This yields a sharper jet-gap-jet signal excess at $N_{\text {tracks }}=0$ for large jet distance parameter. At small distance parameter $R$, there is more spillage of charged
TABLE IV. Measured values of the fraction of color-singlet exchange events $f_{\mathrm{CSE}}$ in bins of the azimuthal angular difference between the two leading jets $\Delta \phi_{\mathrm{jj}}$. The first column indicates the $\Delta \phi_{\mathrm{jj}}$ bin intervals and the last column represents the measured fraction. The first and second uncertainties correspond to the statistical and systematic components, respectively. The results are integrated over the allowed $p_{\mathrm{T}}^{\mathrm{jet} 2}$ and $\Delta \eta_{\mathrm{jj}}$ values. The mean values of $\Delta \phi_{\mathrm{jj}}$ in the bin are given in the middle column.

\begin{tabular}{lcc}
\hline \hline$\Delta \phi_{\mathrm{jj}}$ & $\left\langle\Delta \phi_{\mathrm{jj}}\right\rangle$ & $f_{\mathrm{CSE}}[\%]$ \\
\hline $0.00-1.00$ & 0.60 & $0.54 \pm 0.11_{-0.10}^{+0.09}$ \\
$1.00-2.00$ & 1.64 & $0.40 \pm 0.04_{-0.06}^{+0.06}$ \\
$2.00-2.25$ & 2.14 & $0.41 \pm 0.04_{-0.08}^{+0.08}$ \\
$2.25-2.50$ & 2.36 & $0.38 \pm 0.03_{-0.07}^{+0.06}$ \\
$2.50-2.75$ & 2.62 & $0.40 \pm 0.02_{-0.06}^{+0.05}$ \\
$2.75-3.00$ & 2.86 & $0.57 \pm 0.02_{-0.09}^{+0.07}$ \\
$3.00-\pi$ & 3.06 & $1.03 \pm 0.02_{-0.15}^{+0.14}$ \\
\hline \hline
\end{tabular}

particles into the gap region, since the jet axes can approach the edge of the $|\eta|<1$ interval more closely. The shape of the multiplicity distribution of color-exchange dijet events remains mostly unaffected by the size of $R$. In these

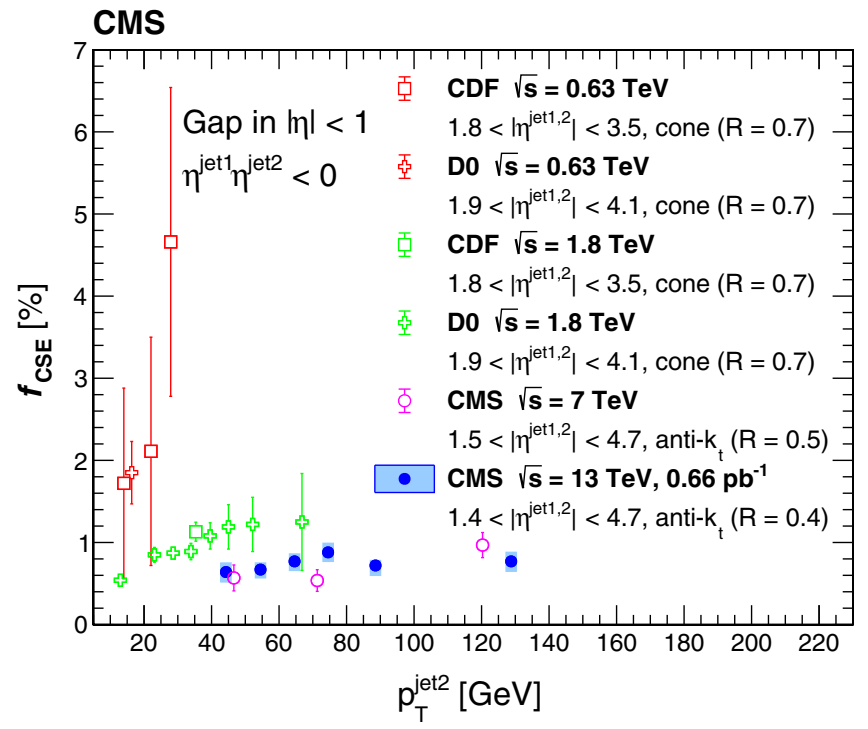

FIG. 9. Fraction of color-singlet exchange dijet events, $f_{\mathrm{CSE}}$, measured as a function of $p_{\mathrm{T}}^{\text {jet} 2}$ by the D0 and CDF Collaborations $[43,45,46]$ at $\sqrt{s}=0.63$ (red open symbols) and $1.8 \mathrm{TeV}$ (green open symbols), by the CMS Collaboration [47] at $7 \mathrm{TeV}$ (magenta open symbols), and the present results at $13 \mathrm{TeV}$ (filled circles). The vertical bars of the open symbols represent the total experimental uncertainties. The vertical bars of the $13 \mathrm{TeV}$ measurement represent the statistical uncertainties, and boxes represent the combination of statistical and systematic uncertainties in quadrature. The central gap is defined by means of the particle activity in the $|\eta|<1$ interval in these measurements, as described in the text. The jet $p_{\mathrm{T}}$ and $\eta$ requirements of the previous measurements are specified in the legend of the plot. No phase space extrapolations are made in plotting this figure. 
simulation studies, these effects are negligible provided that $f_{\text {CSE }}$ is extracted over the first multiplicity bins $N_{\text {tracks }}<3$, as is done in this measurement.

The study by the D0 Collaboration [43] uses the calorimeter tower multiplicity distribution in $|\eta|<1$, where each calorimeter tower has transverse energy $E_{\mathrm{T}}>200 \mathrm{MeV}$. The 0.63 and $1.8 \mathrm{TeV}$ studies consider jets with $E_{\mathrm{T}}{ }^{\text {jet }}>12 \mathrm{GeV}$ and $1.9<\left|\eta^{\text {jet }}\right|<4$. 1 . The CDF Collaboration measured jet-gap-jet events at 0.63 and $1.8 \mathrm{TeV}[45,46]$. The $N_{\text {tracks }}$ value in the region $|\eta|<1$ with $E_{\mathrm{T}}>300 \mathrm{MeV}$ is used in the CDF analyses. Each of the two leading jets has $1.8<\left|\eta^{\text {jet }}\right|<3.5$, with $E_{\mathrm{T}}{ }^{\text {jet }}>$ $8 \mathrm{GeV}$ and $>20 \mathrm{GeV}$ for the 0.63 and $1.8 \mathrm{TeV}$ studies, respectively. The jets are clustered using the cone algorithm with $R=0.7$ for both CDF and D0 studies. The measurement by CMS at $7 \mathrm{TeV}$ is done in three bins of $p_{\mathrm{T}}^{\mathrm{jet} 2}=40-60,60-100$, and 100-200 GeV [47]. The jets are clustered with the anti- $k_{\mathrm{T}}$ algorithm with $R=0.5$ in the previous CMS study. Each of the two leading jets has $1.5<\left|\eta^{\mathrm{jet}}\right|<4.7$, and the signal extraction is based on the $N_{\text {tracks }}$ distribution with $p_{\mathrm{T}}>200 \mathrm{MeV}$ in $|\eta|<1$.

In Fig. 9, the D0 and CDF Collaborations find that $f_{\mathrm{CSE}}$ decreases by a factor of $2.5 \pm 0.9$ [43] and $3.4 \pm 1.2$ [46], respectively, when $\sqrt{s}$ increases from 0.63 to $1.8 \mathrm{TeV}$. Similarly, the results by the CMS experiment at $7 \mathrm{TeV}$ show a $f_{\mathrm{CSE}}$ that decreases by a factor of around 2 with respect to the $1.8 \mathrm{TeV}$ results at the Tevatron [47]. The observed energy dependence of the previous measurements is generally attributed to a larger number of soft parton interactions with increasing $\sqrt{s}$, which enhances the probability of the gap being destroyed. The $13 \mathrm{TeV}$ results show there is no further decrease of the $f_{\mathrm{CSE}}$ values relative to the $7 \mathrm{TeV}$ results, within the uncertainties. This could be an indication that the rapidity gap survival probability stops decreasing at the center-of-mass energies probed at the LHC for the jet-gap-jet process.

The present measurement of $f_{\mathrm{CSE}}$ expands the reach in $\Delta \eta_{\mathrm{jj}}$ covered in the earlier $7 \mathrm{TeV}$ CMS measurement [47], as seen in Fig. 10. The measurement of $f_{\mathrm{CSE}}$ as a function of $\Delta \eta_{\mathrm{jj}}$ at $7 \mathrm{TeV}$ is carried out in three bins of $\Delta \eta_{\mathrm{jj}}=3-4$, 4-5, and 5-7 units for each bin of $p_{\mathrm{T}}^{\mathrm{jet} 2}$. The dependence of $f_{\mathrm{CSE}}$ as a function of $\Delta \eta_{\mathrm{jj}}$ at $13 \mathrm{TeV}$ confirms the trend observed by CMS at $7 \mathrm{TeV}$ and extends the range previously explored toward large values of $6.5<\Delta \eta_{\mathrm{jj}}<8$.

\section{B. Results for jet-gap-jet events with an intact proton}

The fraction $f_{\mathrm{CSE}}$ in events with intact protons is $f_{\mathrm{CSE}}=$ $\left[1.92 \pm 0.46(\text { stat })_{-0.62}^{+0.69}\right.$ (syst) $] \%$. Although the dijet events with an intact proton cover the same phase space as those in the inclusive dijet analysis, most of the events used in the study populate the regions $3.0<\Delta \eta_{\mathrm{jj}}<6.5$ and $40<$ $p_{\mathrm{T}}^{\text {jet2 }}<100 \mathrm{GeV}$ because of the limited sample size of

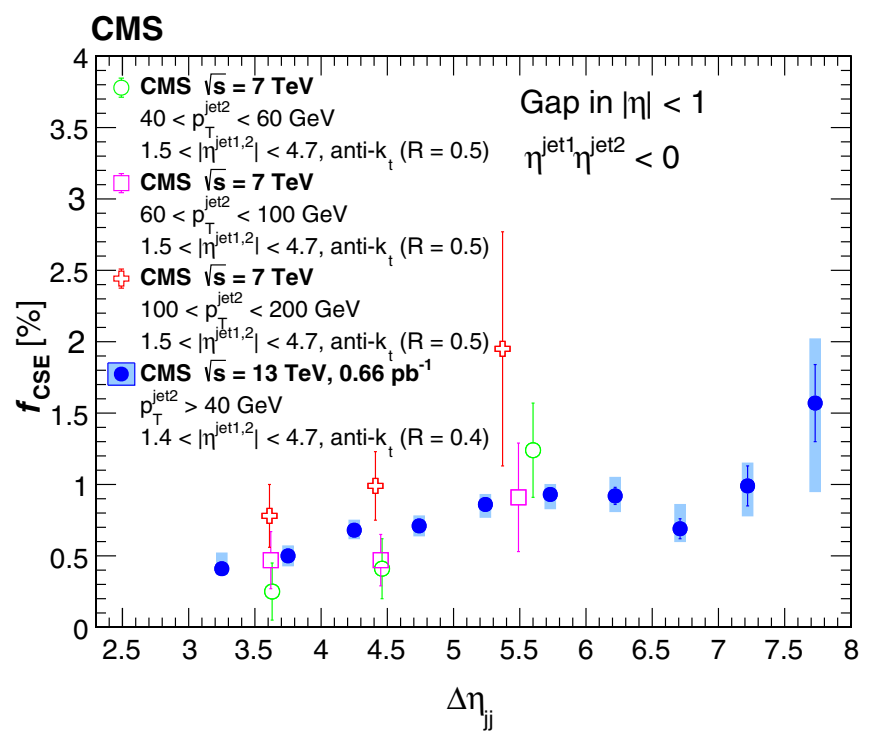

FIG. 10. Fraction of color-singlet exchange dijet events, $f_{\mathrm{CSE}}$, measured as a function of $\Delta \eta_{\mathrm{jj}}$ by CMS at $7 \mathrm{TeV}$ [47] and the present measurement at $13 \mathrm{TeV}$. The $7 \mathrm{TeV}$ measurement was performed in three bins of $p_{\mathrm{T}}^{\mathrm{jet} 2}=40-60,60-100$, and 100$200 \mathrm{GeV}$, which are represented by the open circle, open square, and open cross symbols, respectively. The present $13 \mathrm{TeV}$ results are represented by the filled circles. The vertical bars of the $7 \mathrm{TeV}$ measurement represent the total experimental uncertainties. The vertical bars of the $13 \mathrm{TeV}$ measurement represent the statistical uncertainties, and boxes represent the combination of statistical and systematic uncertainties in quadrature.

events with intact protons. The fraction $f_{\mathrm{CSE}}$ in events with an intact proton is $2.91 \pm 0.70$ (stat) ${ }_{-1.01}^{+1.08}$ (syst) times larger than that extracted for inclusive dijet production, where the two leading jets have similar kinematics to events with an intact proton, i.e., $40<p_{\mathrm{T}}^{\text {jet2 }}<100 \mathrm{GeV}$ and $3.0<\Delta \eta_{\mathrm{jj}}<$ 6.5 for jet-gap-jet events considered in the aforementioned double ratio calculation. The $f_{\mathrm{CSE}}$ ratio in the latter jet-gap-jet subsample has a value of $f_{\mathrm{CSE}}=[0.66 \pm$ 0.01 (stat $)_{-0.09}^{+0.06}$ (syst)]\%. Correlations of systematic uncertainties associated with jet reconstruction and central gap definition are included when evaluating the uncertainties in the double ratios. Statistical and systematic uncertainties in the double ratio are largely dominated by the uncertainties in the CMS-TOTEM $f_{\text {CSE }}$ measurement. The CMSTOTEM results, when compared with the CMS results extracted in inclusive dijet production, suggest that the relative abundance of dijet events with a central gap is larger in events with an intact proton. This is illustrated in Fig. 11, where the results for $f_{\mathrm{CSE}}$ are presented as a function of $\Delta \eta_{\mathrm{jj}}$ and $p_{\mathrm{T}}^{\mathrm{jet} 2}$.

The larger $f_{\mathrm{CSE}}$ value in events with an intact proton may reflect a reduced spectator parton activity in reactions with an intact proton in comparison to the soft parton activity present in interactions where the proton breaks up. In the latter, there can be soft parton exchanges between the 

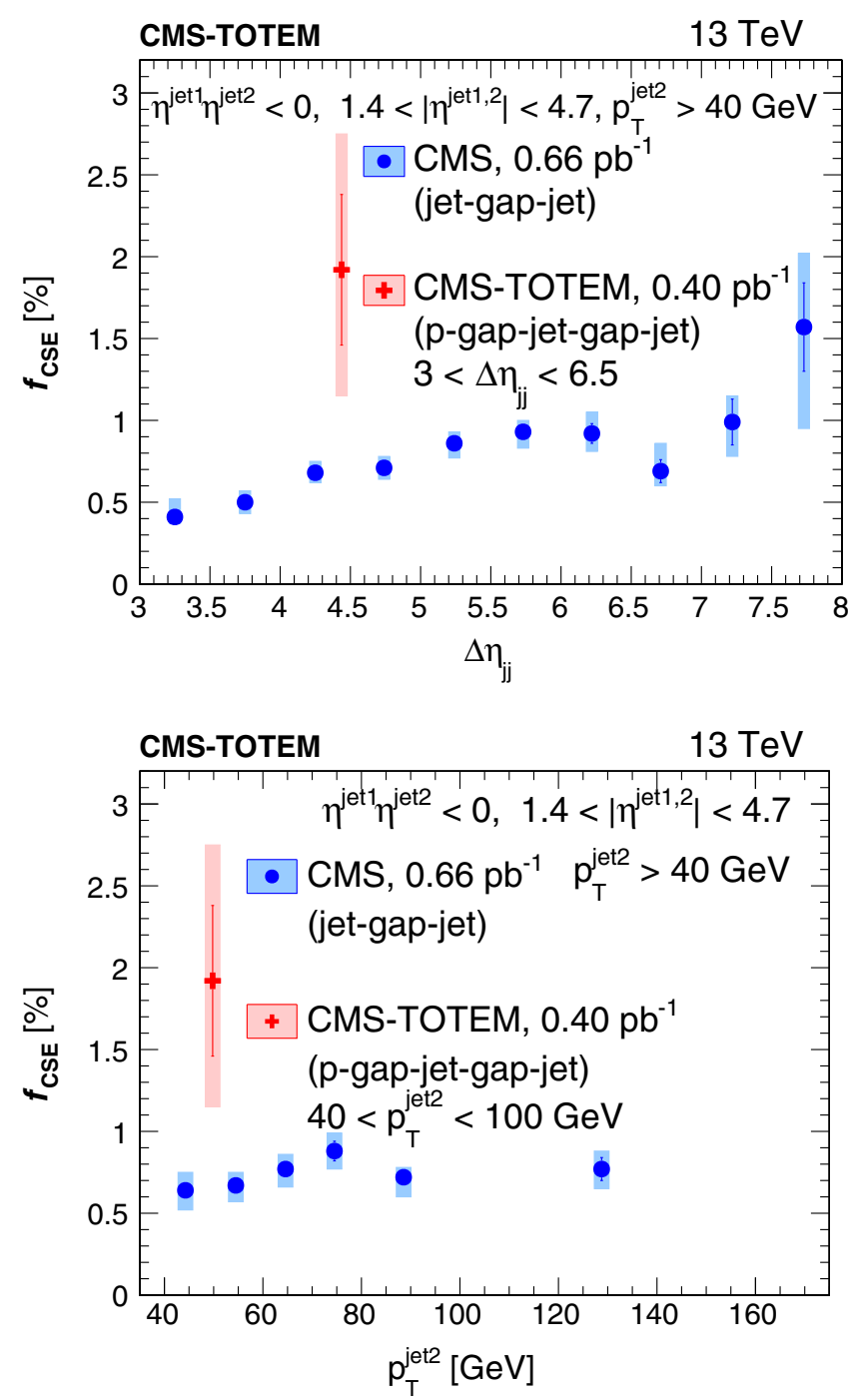

FIG. 11. Fraction of hard color-singlet exchange dijet events $f_{\mathrm{CSE}}$, measured as a function of $\Delta \eta_{\mathrm{jj}}$ (upper) and $p_{\mathrm{T}}^{\text {jet2 }}$ (lower) extracted in inclusive dijet event production (labeled CMS, represented by the blue circle markers) and in dijet events with an intact proton at $13 \mathrm{TeV}$ (labeled CMS-TOTEM, represented by the red cross marker). The vertical bars represent the statistical uncertainties, and boxes represent the combination of statistical and systematic uncertainties in quadrature. The CMS results are plotted at the mean values of $\Delta \eta_{\mathrm{jj}}$ and $p_{\mathrm{T}}^{\mathrm{jet} 2}$ in the bin. Similarly, the CMS-TOTEM result is plotted at the mean value of $\Delta \eta_{\mathrm{jj}}$ and $p_{\mathrm{T}}^{\mathrm{jet} 2}$ in the CMS-TOTEM combined sample. The $40<p_{\mathrm{T}}^{\mathrm{jet} 2}<$ $100 \mathrm{GeV}$ and $3.0<\Delta \eta_{\mathrm{jj}}<6.5$ ranges below the CMS-TOTEM legend represent the dijet phase space covered by events with an intact proton with the present sample size, rather than a selection requirement, as described in the text.

proton remnants and partons produced in the collision, which can destroy the central gap signature between the final-state jets. A similar effect has been observed in other diffractive topologies in dijet events with two rapidity gaps by the CDF Collaboration at $\sqrt{s}=1.8 \mathrm{TeV}$ [103]. In the CDF measurement, comparisons are made of: (i) the ratio of yields of single-diffractive dijet events to nondiffractive dijet events, $R_{\mathrm{ND}}^{\mathrm{SD}}$, and (ii) the ratio of double-pomeron exchange dijet events to single-diffractive dijet events, $R_{\mathrm{SD}}^{\mathrm{DPE}}$. CDF finds that the double ratio has a value of $R_{\mathrm{ND}}^{\mathrm{SD}} / R_{\mathrm{SD}}^{\mathrm{DPE}}=0.19 \pm 0.07$ [103]. An analogous double ratio for the present measurement is $f_{\mathrm{CSE}}$ (jet-gap-jet)/ $f_{\text {CSE }}($ p-gap-jet-gap-jet $)=0.34 \pm 0.08(\text { stat })_{-0.13}^{+0.12}$ (syst $)$, which is similar to that for the double-pomeron exchange dijet topology reported by CDF. The present results further suggest that a gap is more likely to form or survive in the presence of another gap.

\section{SUMMARY}

Events with two leading jets separated by a large pseudorapidity $(\eta)$ gap have been studied in proton-proton $(p p)$ collisions at $\sqrt{s}=13 \mathrm{TeV}$ with the CMS and TOTEM experiments at the CERN LHC in 2015. The pseudorapidity gap is defined by the absence of charged particles with transverse momentum $p_{\mathrm{T}}>200 \mathrm{MeV}$ in the $|\eta|<1$ region. Each of the two leading $p_{\mathrm{T}}$ jets has $1.4<\left|\eta^{\mathrm{jet}}\right|<4.7$ and $p_{\mathrm{T}}^{\text {jet }}>40 \mathrm{GeV}$, with $\eta^{\text {jet1 }} \eta^{\text {jet2 }}<0$, where jet1 and jet2 are the leading and subleading jets in $p_{\mathrm{T}}$. The pseudorapidity gap signature is assumed to be caused by hard color-singlet exchange, which is described in terms of two-gluon exchange in perturbative quantum chromodynamics. Color-singlet exchange events appear as an excess of events over the expected charged particle multiplicity contribution from color-exchange dijet events at the lowest charged particle multiplicity. The ratio of color-singlet exchange events to all dijet events, $f_{\mathrm{CSE}}$, has been measured as a function of $p_{\mathrm{T}}^{\mathrm{jet} 2}$, the $\eta$ difference between the two leading jets, $\Delta \eta_{\mathrm{jj}} \equiv\left|\eta^{\mathrm{jet} 1}-\eta^{\mathrm{jet} 2}\right|$, and the azimuthal angular separation between the two leading jets, $\Delta \phi_{\mathrm{jj}} \equiv\left|\phi^{\text {jet1 }}-\phi^{\text {jet2 }}\right|$.

The measured $f_{\mathrm{CSE}}$ values are in the range of $0.4-1.0 \%$. The ratio $f_{\text {CSE }}$ increases with $\Delta \eta_{\mathrm{jj}}$, has a weak dependence on $p_{\mathrm{T}}^{\mathrm{jet} 2}$, and increases as $\Delta \phi_{\mathrm{jj}}$ approaches $\pi$. No significant difference in $f_{\mathrm{CSE}}$ is observed between the $13 \mathrm{TeV}$ results and those presented by the CMS Collaboration at $7 \mathrm{TeV}$. This is in contrast to the trend found at lower energies of 0.63 and $1.8 \mathrm{TeV}$ by the D0 and CDF Collaborations, where a significant decrease of $f_{\mathrm{CSE}}$ with increasing $\sqrt{s}$ was observed, as illustrated in Fig. 9. The results are compared with calculations based on the Balitsky-FadinKuraev-Lipatov framework [3-5] with resummation of large logarithms of energy at next-to-leading logarithmic accuracy using leading order impact factors, and various treatments of gap survival probability effects. The implementation by Royon, Marquet, and Kepka [54,55] describes some features of the data, but is not able to simultaneously describe all aspects of the measurements. The implementation by Ekstedt, Enberg, Ingelman, and Motyka $[53,56]$ gives a fair description of the data in $\Delta \eta_{\mathrm{jj}}$ and $p_{\mathrm{T}}^{\mathrm{jet} 2}$ within the uncertainties only when considering 
survival probability effects based on multiple-parton interactions and their soft color interaction model.

In addition, a sample of dijet events with intact protons collected by the CMS and TOTEM experiments is used to study jet-gap-jet events with intact protons, which correspond to proton-gap-jet-gap-jet topologies. This is the first analysis of this diffractive event topology. The $f_{\text {CSE }}$ value extracted in this sample is $2.91 \pm 0.70$ (stat $)_{-1.01}^{+1.08}$ (syst) times larger than that found in inclusive dijet production, suggesting a larger abundance of jets with central gaps in events with detected intact protons. This can be interpreted in terms of a lower spectator parton activity in events with intact protons, which decreases the likelihood of the central gap signature being spoiled.

\section{ACKNOWLEDGMENTS}

We thank Andreas Ekstedt, Rikard Enberg, Gunnar Ingelman, Leszek Motyka and Cyrille Marquet, Oldrich Kepka for providing the BFKL predictions of their respective models. We congratulate our colleagues in the CERN accelerator departments for the excellent performance of the LHC and thank the technical and administrative staffs at CERN and at other CMS and TOTEM institutes for their contributions to the success of the CMS-TOTEM effort. In addition, we gratefully acknowledge the computing centers and personnel of the Worldwide LHC Computing Grid and other centers for delivering so effectively the computing infrastructure essential to our analyses. Finally, we acknowledge the enduring support for the construction and operation of the LHC, the CMS and TOTEM detectors, and the supporting computing infrastructure provided by the following funding agencies: $\mathrm{BMBWF}$ and FWF (Austria); FNRS and FWO (Belgium); CNPq, CAPES, FAPERJ, FAPERGS, and FAPESP (Brazil); MES (Bulgaria); CERN; CAS, MoST, and NSFC (China); COLCIENCIAS (Colombia); MSES and CSF (Croatia); RIF (Cyprus); SENESCYT (Ecuador); MoER, ERC PUT and ERDF (Estonia); Academy of Finland, Finnish Academy of Science and Letters (The Vilho Yrjö and Kalle Väisälä Fund), MEC, Magnus Ehrnrooth Foundation, HIP, and Waldemar von Frenckell Foundation (Finland); CEA and CNRS/IN2P3 (France); BMBF, DFG, and HGF (Germany); GSRT (Greece); the Circles of Knowledge Club and NKFIA (Hungary); DAE and DST (India); IPM (Iran); SFI (Ireland); INFN (Italy); MSIP and NRF (Republic of Korea); MES (Latvia); LAS (Lithuania); MOE and UM (Malaysia); BUAP, CINVESTAV, CONACYT, LNS, SEP, and UASLP-FAI (Mexico); MOS (Montenegro); MBIE (New Zealand); PAEC (Pakistan); MSHE and NSC (Poland); FCT (Portugal); JINR (Dubna); MON, RosAtom, RAS, RFBR, and NRC KI (Russia); MESTD (Serbia); SEIDI, CPAN, PCTI, and FEDER (Spain); MOSTR (Sri Lanka); Swiss Funding Agencies (Switzerland); MST (Taipei); ThEPCenter, IPST, STAR, and NSTDA (Thailand); TUBITAK and
TAEK (Turkey); NASU (Ukraine); STFC (United Kingdom); DOE and NSF (USA). Individuals have received support from the Marie-Curie program and the European Research Council and Horizon 2020 Grant, Contract No. 675440, No. 724704, No. 752730, and No. 765710 (European Union); the Leventis Foundation; the Alfred P. Sloan Foundation; the Alexander von Humboldt Foundation; the Belgian Federal Science Policy Office; the Fonds pour la Formation à la Recherche dans l'Industrie et dans l'Agriculture (FRIABelgium); the Agentschap voor Innovatie door Wetenschap en Technologie (IWT-Belgium); the F. R. S.-FNRS and FWO (Belgium) under the "Excellence of ScienceEOS"-be.h Project No. 30820817; the Beijing Municipal Science \& Technology Commission, No. Z191100007219010; the Ministry of Education, Youth and Sports (MEYS) and MSMT CR of the Czech Republic; the Nylands nation vid Helsingfors universitet (Finland); the Deutsche Forschungsgemeinschaft (DFG), under Germany's Excellence Strategy-EXC 2121 "Quantum Universe"-390833306, and under Project No 400140256-GRK2497; the Lendület ("Momentum") Program and the János Bolyai Research Scholarship of the Hungarian Academy of Sciences, the New National Excellence Program ÚNKP, the NKFIA research Grants No. 123842 , No. 123959 , No. 124845, No. 124850 , No. 125105 , No. 128713 , No. 128786, No. 129058 , No. K 133046, and EFOP-3.6.1-16-2016-00001 (Hungary); the Council of Science and Industrial Research, India; the HOMING PLUS program of the Foundation for Polish Science, cofinanced from European Union, Regional Development Fund, the Mobility Plus program of the Ministry of Science and Higher Education, including Grant No. MNiSW DIR/WK/2018/13, the National Science Center (Poland), Contracts Harmonia No. 2014/14/M/ST2/00428, Opus No. 2014/13/B/ST2/02543, No. 2014/15/B/ST2/03998, and No. 2015/19/B/ST2/02861, Sonata-bis No. 2012/07/ E/ST2/01406; the National Priorities Research Program by Qatar National Research Fund; the Ministry of Science and Higher Education, Project No. 0723-20200041 (Russia); the Tomsk Polytechnic University Competitiveness Enhancement Program; the Programa Estatal de Fomento de la Investigación Científica y Técnica de Excelencia María de Maeztu, Grant No. MDM-2015-0509 and the Programa Severo Ochoa del Principado de Asturias; the Thalis and Aristeia programs cofinanced by EU-ESF and the Greek NSRF; the Rachadapisek Sompot Fund for Postdoctoral Fellowship, Chulalongkorn University and the Chulalongkorn Academic into Its 2nd Century Project Advancement Project (Thailand); the Kavli Foundation; the Nvidia Corporation; the SuperMicro Corporation; the Welch Foundation, Contract No. C-1845; and the Weston Havens Foundation (USA). 
[1] P. A. Zyla et al. (Particle Data Group), Review of particle physics, Prog. Theor. Exp. Phys. 2020, 083 C01 (2020).

[2] K. Akiba, M. Akbiyik, M. Albrow, M. Arneodo, V. Avati, J. Baechler, O. V. Baillie, P. Bartalini, J. Bartels, S. Baur et al., LHC forward physics, J. Phys. G 43, 110201 (2016).

[3] E. A. Kuraev, L. N. Lipatov, and V. S. Fadin, The Pomeranchuk singularity in nonabelian gauge theories, Sov. Phys. JETP 45, 199 (1977).

[4] I. I. Balitsky and L. N. Lipatov, The Pomeranchuk singularity in quantum chromodynamics, Sov. J. Nucl. Phys. 28, 822 (1978).

[5] L. N. Lipatov, The bare pomeron in quantum chromodynamics, Sov. Phys. JETP 63, 904 (1986).

[6] V. S. Fadin and L. N. Lipatov, BFKL pomeron in the nextto-leading approximation, Phys. Lett. B 429, 127 (1998).

[7] M. Ciafaloni and G. Camici, Energy scale(s) and next-toleading BFKL equation, Phys. Lett. B 430, 349 (1998).

[8] CMS Collaboration, Azimuthal decorrelation of jets widely separated in rapidity in pp collisions at $\sqrt{s}=7 \mathrm{TeV}$, J. High Energy Phys. 08 (2016) 139.

[9] ATLAS Collaboration, Measurement of dijet production with a veto on additional central jet activity in pp collisions at $\sqrt{s}=7 \mathrm{TeV}$ using the ATLAS detector, J. High Energy Phys. 09 (2011) 053.

[10] CMS Collaboration, Ratios of dijet production cross sections as a function of the absolute difference in rapidity between jets in proton-proton collisions at $\sqrt{s}=7 \mathrm{TeV}$, Eur. Phys. J. C 72, 2216 (2012).

[11] LHCb Collaboration, Updated measurements of exclusive $J / \Psi$ and $\psi(2 S)$ production cross sections in pp collisions at $\sqrt{s}=7 \mathrm{TeV}$, J. Phys. G 41, 055002 (2014).

[12] LHCb Collaboration, Measurement of the exclusive $\Upsilon$ production cross section in pp collisions at $\sqrt{s}=7 \mathrm{TeV}$ and $8 \mathrm{TeV}$, J. High Energy Phys. 09 (2015) 084.

[13] ALICE Collaboration, Coherent $J / \Psi$ photoproduction in ultraperipheral $\mathrm{Pb}-\mathrm{Pb}$ collisions at $\sqrt{s_{\mathrm{NN}}}=2.76 \mathrm{TeV}$, Phys. Lett. B 718, 1273 (2013).

[14] ALICE Collaboration, Exclusive $J / \Psi$ Photoproduction off Protons in Ultraperipheral $\mathrm{p}-\mathrm{Pb}$ Collisions at $\sqrt{s_{\mathrm{NN}}}=5.02 \mathrm{TeV}$, Phys. Rev. Lett. 113, 232504 (2014).

[15] ALICE Collaboration, Coherent $\rho^{0}$ photoproduction in ultraperipheral $\mathrm{Pb}-\mathrm{Pb}$ collisions at $\sqrt{s_{\mathrm{NN}}}=2.76 \mathrm{TeV}$, J. High Energy Phys. 09 (2015) 095.

[16] CMS Collaboration, Measurement of exclusive $\Upsilon$ photoproduction from protons in $\mathrm{pPb}$ collisions at $\sqrt{s_{\mathrm{NN}}}=5.02 \mathrm{TeV}$, Eur. Phys. J. C 79, 277 (2019).

[17] CMS Collaboration, Measurement of exclusive $\rho(770)^{0}$ photoproduction in ultraperipheral $\mathrm{pPb}$ collisions at $\sqrt{s_{\mathrm{NN}}}=5.02 \mathrm{TeV}$, Eur. Phys. J. C 79, 702 (2019).

[18] I. Bautista, A. Fernández Téllez, and M. Hentschinski, BFKL evolution and the growth with energy of exclusive $J / \psi$ and $\Upsilon$ photoproduction cross sections, Phys. Rev. D 94, 054002 (2016).

[19] A. Arroyo García, M. Hentschinski, and K. Kutak, QCD evolution based evidence for the onset of gluon saturation in exclusive photo-production of vector mesons, Phys. Lett. B 795, 569 (2019).

[20] V. N. Gribov and L. N. Lipatov, Deep inelastic ep scattering in perturbation theory, Sov. J. Nucl. Phys. 15, 438 (1972).
[21] G. Altarelli and G. Parisi, Asymptotic freedom in parton language, Nucl. Phys. B126, 298 (1977).

[22] Y. L. Dokshitzer, Calculation of the structure functions for deep inelastic scattering and $e^{+} e^{-}$annihilation by perturbation theory in quantum chromodynamics, Sov. Phys. JETP 46, 641 (1977).

[23] CMS Collaboration, Measurement of the differential dijet production cross section in proton-proton collisions at $\sqrt{s}=7 \mathrm{TeV}$, Phys. Lett. B 700, 187 (2011).

[24] CMS Collaboration, Measurement of the inclusive production cross sections for forward jets and for dijet events with one forward and one central jet in pp collisions at $\sqrt{s}=7 \mathrm{TeV}$, J. High Energy Phys. 06 (2012) 036.

[25] CMS Collaboration, Measurement of the inclusive jet cross section in pp collisions at $\sqrt{s}=2.76 \mathrm{TeV}$, Eur. Phys. J. C 76, 265 (2016).

[26] CMS Collaboration, Measurement of dijet azimuthal decorrelation in pp collisions at $\sqrt{s}=8 \mathrm{TeV}$, Eur. Phys. J. C 76, 536 (2016).

[27] CMS Collaboration, Measurement of the doubledifferential inclusive jet cross section in proton-proton collisions at $\sqrt{s}=13 \mathrm{TeV}$, Eur. Phys. J. C 76, 451 (2016).

[28] CMS Collaboration, Measurement and QCD analysis of double-differential inclusive jet cross sections in pp collisions at $\sqrt{s}=8 \mathrm{TeV}$ and cross section ratios to 2.76 and 7 TeV, J. High Energy Phys. 03 (2017) 156.

[29] CMS Collaboration, Azimuthal correlations for inclusive 2-jet, 3-jet, and 4-jet events in pp collisions at $\sqrt{s}=13 \mathrm{TeV}$, Eur. Phys. J. C 78, 566 (2018).

[30] CMS Collaboration, Measurement of inclusive very forward jet cross sections in proton-lead collisions at $\sqrt{s_{\mathrm{NN}}}=5.02 \mathrm{TeV}$, J. High Energy Phys. 05 (2019) 043.

[31] ATLAS Collaboration, Measurement of inclusive jet and dijet cross sections in proton-proton collisions at $7 \mathrm{TeV}$ centre-of-mass energy with the ATLAS detector, Eur. Phys. J. C 71, 1512 (2011).

[32] ATLAS Collaboration, Measurement of inclusive jet and dijet production in pp collisions at $\sqrt{s}=7 \mathrm{TeV}$ using the ATLAS detector, Phys. Rev. D 86, 014022 (2012).

[33] ATLAS Collaboration, Measurement of dijet cross sections in pp collisions at $7 \mathrm{TeV}$ centre-of-mass energy using the ATLAS detector, J. High Energy Phys. 05 (2014) 059.

[34] ATLAS Collaboration, Measurements of jet vetoes and azimuthal decorrelations in dijet events produced in $\mathrm{pp}$ collisions at $\sqrt{s}=7 \mathrm{TeV}$ using the ATLAS detector, Eur. Phys. J. C 74, 3117 (2014).

[35] ATLAS Collaboration, Measurement of the inclusive jet cross sections in proton-proton collisions at $\sqrt{s}=8 \mathrm{TeV}$ with the ATLAS detector, J. High Energy Phys. 09 (2017) 020 .

[36] ATLAS Collaboration, Measurement of dijet azimuthal decorrelations in pp collisions at $\sqrt{s}=8 \mathrm{TeV}$ with the ATLAS detector and determination of the strong coupling, Phys. Rev. D 98, 092004 (2018).

[37] R. D. Ball, V. Bertone, M. Bonvini, S. Marzani, J. Rojo, and L. Rottoli, Parton distributions with small- $x$ resummation: Evidence for BFKL dynamics in HERA data, Eur. Phys. J. C 78, 321 (2018).

[38] A. H. Mueller and W. K. Tang, High energy parton-parton elastic scattering in QCD, Phys. Lett. B 284, 123 (1992). 
[39] C. Adloff et al. (H1 Collaboration), Energy flow and rapidity gaps between jets in photoproduction at HERA, Eur. Phys. J. C 24, 517 (2002).

[40] M. Derrick et al. (ZEUS Collaboration), Rapidity gaps between jets in photoproduction at HERA, Phys. Lett. B 369, 55 (1996).

[41] S. Abachi et al. (D0 Collaboration), Rapidity Gaps Between Jets in $\mathrm{p} \overline{\mathrm{p}}$ Collisions at $\sqrt{s}=1.8 \mathrm{TeV}$, Phys. Rev. Lett. 72, 2332 (1994).

[42] S. Abachi et al. (D0 Collaboration), Jet Production via Strongly-Interacting Color-Singlet Exchange in $\mathrm{p} \overline{\mathrm{p}}$ Collisions, Phys. Rev. Lett. 76, 734 (1996).

[43] B. Abbott et al. (D0 Collaboration), Probing hard colorsinglet exchange in $\mathrm{p} \overline{\mathrm{p}}$ collisions at $\sqrt{s}=630 \mathrm{GeV}$ and $1800 \mathrm{GeV}$, Phys. Lett. B 440, 189 (1998).

[44] F. Abe et al. (CDF Collaboration), Observation of Rapidity Gaps in $\bar{p}$ p Collisions at 1.8 TeV, Phys. Rev. Lett. 74, 855 (1995).

[45] F. Abe et al. (CDF Collaboration), Dijet Production by Color-Singlet Exchange at the Fermilab Tevatron, Phys. Rev. Lett. 80, 1156 (1998).

[46] F. Abe et al. (CDF Collaboration), Events with a Rapidity Gap Between Jets in $\bar{p}$ p Collisions at $\sqrt{s}=630 \mathrm{GeV}$, Phys. Rev. Lett. 81, 5278 (1998).

[47] CMS Collaboration, Study of dijet events with a large rapidity gap between the two leading jets in pp collisions at $\sqrt{s}=7$ TeV, Eur. Phys. J. C 78, 242 (2018).

[48] J. D. Bjorken, Rapidity gaps and jets as a new-physics signature in very-high-energy hadron-hadron collisions, Phys. Rev. D 47, 101 (1993).

[49] V. Barone and E. Predazzi, High-Energy Particle Diffraction (Springer, Berlin, 2002).

[50] S. Donnachie, G. Dosch, P. Landshoff, and O. Nachtmann, Pomeron Physics and QCD, Cambridge Monographs on Particle Physics, Nuclear Physics and Cosmology (Cambridge University Press, Cambridge, England, 2002).

[51] J. R. Forshaw and D. A. Ross, Quantum Chromodynamics and the Pomeron, Cambridge Lecture Notes in Physics (Cambridge University Press, Cambridge, England, 1997).

[52] B. Cox, J. R. Forshaw, and L. Lönnblad, Hard color-singlet exchange at the Tevatron, J. High Energy Phys. 10 (1999) 023.

[53] R. Enberg, G. Ingelman, and L. Motyka, Hard color-singlet exchange and gaps between jets at the Tevatron, Phys. Lett. B 524, 273 (2002).

[54] F. Chevallier, O. Kepka, C. Marquet, and C. Royon, Gaps between jets at hadron colliders in the next-to-leading BFKL framework, Phys. Rev. D 79, 094019 (2009).

[55] O. Kepka, C. Marquet, and C. Royon, Gaps between jets in hadronic collisions, Phys. Rev. D 83, 034036 (2011).

[56] A. Ekstedt, R. Enberg, and G. Ingelman, Hard color-singlet BFKL exchange and gaps between jets at the LHC, arXiv: 1703.10919.

[57] J. D. Bjorken, A full-acceptance detector for SSC physics at low and intermediate mass scales: An expression of interest to the SSC, Int. J. Mod. Phys. A 07, 4189 (1992).

[58] E. Gotsman, E. Levin, and U. Maor, Energy dependence of the survival probability of large rapidity gaps, Phys. Lett. B 438, 229 (1998).
[59] V. A. Khoze, A. D. Martin, and M. G. Ryskin, Diffraction at the LHC, Eur. Phys. J. C 73, 2503 (2013).

[60] E. Gotsman, E. Levin, and U. Maor, CGC/saturation approach for soft interactions at high energy: Survival probability of central exclusive production, Eur. Phys. J. C 76, 177 (2016).

[61] V. A. Khoze, A. D. Martin, and M. G. Ryskin, Multiple interactions and rapidity gap survival, J. Phys. G 45, 053002 (2018).

[62] I. Babiarz, R. Staszewski, and A. Szczurek, Multi-parton interactions and rapidity gap survival probability in jetgap-jet processes, Phys. Lett. B 771, 532 (2017).

[63] C. Marquet, C. Royon, M. Trzebiński, and R. Žlebčík, Gaps between jets in double-pomeron-exchange processes at the LHC, Phys. Rev. D 87, 034010 (2013).

[64] G. Anelli et al. (TOTEM Collaboration), The TOTEM experiment at the CERN Large Hadron Collider, J. Instrum. 3, S08007 (2008).

[65] O. S. Brüning, P. Collier, P. Lebrun, S. Myers, R. Ostojic, J. Poole, and P. Proudlock, LHC design report, Report No. CERN-2004-003-v-1, 2004.

[66] CMS Collaboration, Description and performance of track and primary-vertex reconstruction with the CMS tracker, J. Instrum. 9, P10009 (2014).

[67] CMS Collaboration, Particle-flow reconstruction and global event description with the CMS detector, J. Instrum. 12, P10003 (2017).

[68] M. Cacciari, G. P. Salam, and G. Soyez, The anti- $k_{T}$ jet clustering algorithm, J. High Energy Phys. 04 (2008) 063.

[69] M. Cacciari, G. P. Salam, and G. Soyez, FastJet user manual, Eur. Phys. J. C 72, 1896 (2012).

[70] CMS Collaboration, Jet energy scale and resolution in the CMS experiment in pp collisions at $8 \mathrm{TeV}$, J. Instrum. 12, P02014 (2017).

[71] CMS Collaboration, The CMS experiment at the CERN LHC, J. Instrum. 3, S08004 (2008).

[72] G. Antchev et al. (TOTEM Collaboration), LHC optics measurement with proton tracks detected by the Roman pots of the TOTEM experiment, New J. Phys. 16, 103041 (2014).

[73] G. Antchev et al. (TOTEM Collaboration), Performance of the TOTEM detectors at the LHC, Int. J. Mod. Phys. A 28, 1330046 (2013).

[74] H. Niewiadomski, Reconstruction of protons in the TOTEM Roman pot detectors at the LHC, Ph.D. thesis, Manchester University, 2008 [Report No. CERN-THESIS-2008-080].

[75] CMS and TOTEM Collaborations, Measurement of singlediffractive dijet production in proton-proton collisions at $\sqrt{s}=8 \mathrm{TeV}$ with the CMS and TOTEM experiments, Eur. Phys. J. C 80, 1164 (2020).

[76] CMS Collaboration, The CMS trigger system, J. Instrum. 12, P01020 (2017).

[77] CMS Collaboration, Observation of a diffractive contribution to dijet production in proton-proton collisions at $\sqrt{s}=7$ TeV, Phys. Rev. D 87, 012006 (2013).

[78] M. Hentschinski, J. D. Madrigal Martínez, B. Murdaca, and A. Sabio Vera, The next-to-leading order vertex for a forward jet plus a rapidity gap at high energies, Phys. Lett. B 735, 168 (2014). 
[79] M. Hentschinski, J. D. Madrigal Martínez, B. Murdaca, and A. Sabio Vera, The gluon-induced Mueller-Tang jet impact factor at next-to-leading order, Nucl. Phys. B889, 549 (2014).

[80] A. Giovannini and L. Van Hove, Negative binomial multiplicity distributions in high energy hadron collisions, Z. Phys. C 30, 391 (1986).

[81] P. Ghosh, Negative binomial multiplicity distribution in proton-proton collisions in limited pseudorapidity intervals at LHC up to $\sqrt{s}=7 \mathrm{TeV}$ and the clan model, Phys. Rev. D 85, 054017 (2012).

[82] G. J. Alner et al. (UA5 Collaboration), Multiplicity distributions in different pseudorapidity intervals at a centerof-mass energy of $540 \mathrm{GeV}$, Phys. Lett. 160B, 193 (1985).

[83] R. E. Ansorge et al. (UA5 Collaboration), Charged particle multiplicity distributions at $200 \mathrm{GeV}$ and $900 \mathrm{GeV}$ centerof-mass energy, Z. Phys. C 43, 357 (1989).

[84] ALICE Collaboration, Charged-particle multiplicities in proton-proton collisions at $\sqrt{s}=0.9$ to $8 \mathrm{TeV}$, Eur. Phys. J. C 77, 33 (2017).

[85] T. Sjöstrand, S. Ask, J. R. Christiansen, R. Corke, N. Desai, P. Ilten, S. Mrenna, S. Prestel, C. O. Rasmussen, and P.Z. Skands, An introduction to PYTHIA 8.2, Comput. Phys. Commun. 191, 159 (2015).

[86] R. D. Ball, V. Bertone, F. Cerutti, L. Del Debbio, S. Forte, A. Guffanti, J. I. Latorre, J. Rojo, and M. Ubiali (NNPDF Collaboration), Unbiased global determination of parton distributions and their uncertainties at NNLO and at LO, Nucl. Phys. B855, 153 (2012).

[87] R. D. Ball, V. Bertone, S. Carrazza, L. Del Debbio, S. Forte, A. Guffanti, N. P. Hartland, and J. Rojo (NNPDF Collaboration), Parton distributions with QED corrections, Nucl. Phys. B877, 290 (2013).

[88] B. Andersson, G. Gustafson, G. Ingelman, and T. Sjöstrand, Parton fragmentation and string dynamics, Phys. Rep. 97, 31 (1983).

[89] CMS Collaboration, Event generator tunes obtained from underlying event and multiparton scattering measurements, Eur. Phys. J. C 76, 155 (2016).

[90] G. Corcella, I. G. Knowles, G. Marchesini, S. Moretti, K. Odagiri, P. Richardson, M. H. Seymour, and B. R. Webber, HERWIG 6: an event generator for hadron emission reactions with interfering gluons (including supersymmetric processes), J. High Energy Phys. 01 (2001) 010.

[91] J. Pumplin, D. R. Stump, J. Huston, H. L. Lai, P. M. Nadolsky, and W. K. Tung, New generation of parton distributions with uncertainties from global QCD analysis, J. High Energy Phys. 07 (2002) 012.

[92] B. R. Webber, A QCD model for jet fragmentation including soft gluon interference, Nucl. Phys. B238, 492 (1984).

[93] J. M. Butterworth, J. R. Forshaw, and M. H. Seymour, Multiparton interactions in photoproduction at HERA, Z. Phys. C 72, 637 (1996).

[94] S. Agostinelli et al. (GEANT4 Collaboration), GEANT4A simulation toolkit, Nucl. Instrum. Methods Phys. Res., Sect. A 506, 250 (2003).

[95] B. Abbott et al. (D0 Collaboration), Hard single diffraction in $\bar{p} p$ collisions at $\sqrt{s}=630 \mathrm{GeV}$ and $1800 \mathrm{GeV}$, Phys. Lett. B 531, 52 (2002).

[96] T. Affolder et al. (CDF Collaboration), Diffractive Dijets with a Leading Antiproton in $\bar{p} p$ Collisions at $\sqrt{s}=1800 \mathrm{GeV}$, Phys. Rev. Lett. 84, 5043 (2000).

[97] D. Acosta et al. (CDF Collaboration), Diffractive Dijet Production at $\sqrt{s}=630 \mathrm{GeV}$ and $1800 \mathrm{GeV}$ at the Fermilab Tevatron, Phys. Rev. Lett. 88, 151802 (2002).

[98] T. Aaltonen et al. (CDF Collaboration), Observation of exclusive dijet production at the Fermilab Tevatron $\bar{p} p$ Collider, Phys. Rev. D 77, 052004 (2008).

[99] T. Aaltonen et al. (CDF Collaboration), Diffractive dijet production in $\bar{p} p$ collisions at $\sqrt{s}=1.96 \mathrm{TeV}$, Phys. Rev. D 86, 032009 (2012).

[100] V. Andreev et al. (H1 Collaboration), Diffractive dijet production with a leading proton in e $p$ collisions at HERA, J. High Energy Phys. 05 (2015) 056.

[101] P. Świerska and M. Trzebiński, BFKL amplitude parametrization for the jet-gap-jet events at the LHC energies, Acta Phys. Pol. B 46, 2477 (2015).

[102] D. Colferai, F. Schwennsen, L. Szymanowski, and S. Wallon, Mueller-Navelet jets at LHC-complete NLL BFKL calculation, J. High Energy Phys. 12 (2010) 026.

[103] T. Affolder et al. (CDF Collaboration), Dijet Production by Double-Pomeron Exchange at the Fermilab Tevatron, Phys. Rev. Lett. 85, 4215 (2000).

A. M. Sirunyan, ${ }^{1, a, \dagger}$ A. Tumasyan, ${ }^{1, \dagger}$ W. Adam, ${ }^{2, \dagger}$ F. Ambrogi, ${ }^{2, \dagger}$ T. Bergauer, ${ }^{2, \dagger}$ M. Dragicevic, ${ }^{2, \dagger}$ J. Erö, ${ }^{2, \dagger}$ A. Escalante Del Valle, ${ }^{2, \dagger}$ R. Frühwirth, ${ }^{2, b, \dagger}$ M. Jeitler, ${ }^{2, b, \dagger}$ N. Krammer, ${ }^{2, \dagger}$ L. Lechner, ${ }^{2, \dagger}$ D. Liko, ${ }^{2, \dagger}$ T. Madlener, ${ }^{2, \dagger}$ I. Mikulec, ${ }^{2, \dagger}$ F. M. Pitters, ${ }^{2, \dagger}$ N. Rad, ${ }^{2, \dagger}$ J. Schieck, ${ }^{2, b, \dagger}$ R. Schöfbeck, ${ }^{2, \dagger}$ M. Spanring, ${ }^{2, \dagger}$ S. Templ, ${ }^{2, \dagger}$ W. Waltenberger, ${ }^{2, \dagger}$ C.-E. Wulz, ${ }^{2, b, \dagger}$ M. Zarucki, ${ }^{2, \dagger}$ V. Chekhovsky, ${ }^{3, \dagger}$ A. Litomin, ${ }^{3, \dagger}$ V. Makarenko, ${ }^{3, \dagger}$ J. Suarez Gonzalez, ${ }^{3, \dagger}$ M. R. Darwish, ${ }^{4, c, \uparrow}$ E. A. De Wolf, ${ }^{4, \dagger}$ D. Di Croce, ${ }^{4, \dagger}$ X. Janssen, ${ }^{4, \dagger}$ T. Kello, ${ }^{4, d, \dagger}$ A. Lelek, ${ }^{4, \dagger}$ M. Pieters, ${ }^{4, \dagger}$ H. Rejeb Sfar, ${ }^{4, \dagger}$ H. Van Haevermaet, ${ }^{4, \dagger}$ P. Van Mechelen, ${ }^{4, \dagger}$ S. Van Putte, ${ }^{4, \dagger}$ N. Van Remortel, ${ }^{4, \dagger}$ F. Blekman, ${ }^{5, \dagger}$ E. S. Bols, ${ }^{5, \dagger}$ S. S. Chhibra, ${ }^{5, \dagger}$ J. D’Hondt, ${ }^{5, \dagger}$ J. De Clercq, ${ }^{5, \dagger}$ D. Lontkovskyi, ${ }^{5, \dagger}$ S. Lowette, ${ }^{5, \dagger}$ I. Marchesini, ${ }^{5, \dagger}$ S. Moortgat, ${ }^{5, \dagger}$ A. Morton, ${ }^{5, \dagger}$ Q. Python, ${ }^{5, \dagger}$ S. Tavernier, ${ }^{5, \dagger}$ W. Van Doninck, ${ }^{5, \dagger}$ P. Van Mulders, ${ }^{5, \dagger}$ D. Beghin, ${ }^{6, \dagger}$ B. Bilin, ${ }^{6, \dagger}$ B. Clerbaux, ${ }^{6, \dagger}$ G. De Lentdecker, ${ }^{6, \dagger}$ H. Delannoy, ${ }^{6, \dagger}$ B. Dorney, ${ }^{6, \dagger}$ L. Favart, ${ }^{6, \dagger}$ A. Grebenyuk, ${ }^{6, \dagger}$ A. K. Kalsi, ${ }^{6, \dagger}$ I. Makarenko, ${ }^{6, \dagger}$ L. Moureaux, ${ }^{6, \dagger}$ L. Pétré, ${ }^{6, \uparrow}$ A. Popov, ${ }^{6, \dagger}$ N. Postiau, ${ }^{6, \dagger}$ E. Starling,${ }^{6, \dagger}$ L. Thomas, ${ }^{6, \dagger}$ C. Vander Velde, ${ }^{6, \dagger}$ P. Vanlaer, ${ }^{6, \dagger}$ D. Vannerom, ${ }^{6, \dagger}$ L. Wezenbeek, ${ }^{6, \dagger}$ T. Cornelis, ${ }^{7, \dagger}$ D. Dobur, ${ }^{7, \dagger}$ M. Gruchala, ${ }^{7, \dagger}$ I. Khvastunov, ${ }^{7, e, \dagger}$ M. Niedziela, ${ }^{7, \dagger}$ C. Roskas, ${ }^{7, \dagger}$ K. Skovpen,,${ }^{7, \dagger}$ M. Tytgat, ${ }^{7, \dagger}$ 
W. Verbeke, ${ }^{7, \dagger}$ B. Vermassen, ${ }^{7, \dagger}$ M. Vit, ${ }^{7, \dagger}$ G. Bruno, ${ }^{8, \dagger}$ F. Bury,${ }^{8, \dagger}$ C. Caputo, ${ }^{8, \dagger}$ P. David, ${ }^{8, \dagger}$ C. Delaere, ${ }^{8, \dagger}$ M. Delcourt,${ }^{8, \dagger}$ I. S. Donertas, ${ }^{8, \dagger}$ A. Giammanco, ${ }^{8, \dagger}$ V. Lemaitre, ${ }^{8, \dagger}$ K. Mondal, ${ }^{8, \dagger}$ J. Prisciandaro, ${ }^{8, \dagger}$ A. Taliercio, ${ }^{8, \dagger}$ M. Teklishyn, ${ }^{8, \dagger}$ P. Vischia, ${ }^{8, \dagger}$ S. Wuyckens, ${ }^{8, \dagger}$ J. Zobec, ${ }^{8, \dagger}$ G. A. Alves, ${ }^{9, \dagger}$ G. Correia Silva, ${ }^{9, \dagger}$ C. Hensel,,${ }^{9, \dagger}$ A. Moraes, ${ }^{9, \dagger}$

W. L. Aldá Júnior, ${ }^{10, \dagger}$ E. Belchior Batista Das Chagas, ${ }^{10, \dagger}$ H. Brandao Malbouisson, ${ }^{10, \uparrow}$ W. Carvalho, ${ }^{10, \dagger}$ J. Chinellato, ${ }^{10, f, \uparrow}$ E. Coelho, ${ }^{10, \uparrow}$ E. M. Da Costa, ${ }^{10, \uparrow}$ G. G. Da Silveira, ${ }^{10, g, \uparrow}$ D. De Jesus Damiao, ${ }^{10, \uparrow}$ S. Fonseca De Souza, ${ }^{10, \uparrow}$ J. Martins, ${ }^{10, \mathrm{~h}, \uparrow}$ D. Matos Figueiredo,${ }^{10, \dagger}$ M. Medina Jaime,${ }^{10, i, \dagger}$ M. Melo De Almeida, ${ }^{10, \dagger}$ C. Mora Herrera, ${ }^{10, \dagger}$ L. Mundim, ${ }^{10, \dagger}$ H. Nogima,${ }^{10, \dagger}$ P. Rebello Teles,${ }^{10, \dagger}$ L. J. Sanchez Rosas, ${ }^{10, \dagger}$ A. Santoro, ${ }^{10, \dagger}$ S. M. Silva Do Amaral, ${ }^{10, \dagger}$ A. Sznajder, ${ }^{10, \dagger}$ M. Thiel, ${ }^{10, \dagger}$ E. J. Tonelli Manganote,${ }^{10, f, \dagger}$ F. Torres Da Silva De Araujo, ${ }^{10, \dagger}$ A. Vilela Pereira, ${ }^{10, \dagger}$ C. A. Bernardes, ${ }^{11 a, \dagger}$ L. Calligaris, ${ }^{11 a, \dagger}$ T. R. Fernandez Perez Tomei, ${ }^{11 a, \dagger}$ E. M. Gregores,${ }^{11 \mathrm{a}, 1 \mathrm{lb}, \dagger}$ D. S. Lemos, ${ }^{11 \mathrm{a}, \dagger}$ P. G. Mercadante, ${ }^{11 \mathrm{a}, 11 \mathrm{~b}, \uparrow}$ S. F. Novaes, ${ }^{11 a, \dagger}$ Sandra S. Padula, ${ }^{11 a, \dagger}$ A. Aleksandrov, ${ }^{12, \dagger}$ R. Hadjiiska, ${ }^{12, \dagger}$ P. Iaydjiev, ${ }^{12, \dagger}$ M. Misheva, ${ }^{12, \dagger}$ M. Rodozov, ${ }^{12, \dagger}$ M. Shopova, ${ }^{12, \dagger}$ G. Sultanov, ${ }^{12, \dagger}$ M. Bonchev, ${ }^{13, \dagger}$ A. Dimitrov, ${ }^{13, \dagger}$ T. Ivanov, ${ }^{13, \dagger}$ L. Litov, ${ }^{13, \dagger}$ B. Pavlov,${ }^{13, \dagger}$ P. Petkov, ${ }^{13, \dagger}$ A. Petrov, ${ }^{13, \dagger}$ W. Fang, ${ }^{14, d, \dagger}$ Q. Guo, ${ }^{14, \dagger}$ H. Wang, ${ }^{14, \dagger}$ L. Yuan, ${ }^{14, \dagger}$ M. Ahmad,${ }^{15, \dagger}$ Z. Hu ${ }^{15, \dagger}$ Y. Wang, ${ }^{15, \dagger}$ E. Chapon, ${ }^{16, \dagger}$ G. M. Chen, ${ }^{16, j, \dagger}$ H. S. Chen, ${ }^{16, j, \dagger}$ M. Chen, ${ }^{16, \dagger}$ D. Leggat,${ }^{16, \dagger}$ H. Liao, ${ }^{16, \dagger}$ Z. Liu, ${ }^{16, \dagger}$ R. Sharma, ${ }^{16, \dagger}$ A. Spiezia, ${ }^{16, \dagger}$ J. Tao, ${ }^{16, \dagger}$ J. Thomas-wilsker ${ }^{16, \dagger}$ J. Wang, ${ }^{16, \dagger}$ H. Zhang, ${ }^{16, \dagger}$ S. Zhang, ${ }^{16, j, \dagger}$ J. Zhao, ${ }^{16, \dagger}$ A. Agapitos, ${ }^{17, \dagger}$ Y. Ban, ${ }^{17, \dagger}$ C. Chen, ${ }^{17, \dagger}$ A. Levin, ${ }^{17, \dagger}$ J. Li, ${ }^{17, \uparrow}$ Q. Li ${ }^{17, \uparrow}$ M. Lu, ${ }^{17, \dagger}$ X. Lyu,${ }^{17, \dagger}$ Y. Mao, ${ }^{17, \dagger}$ S. J. Qian, ${ }^{17, \dagger}$ D. Wang, ${ }^{17, \dagger}$ Q. Wang, ${ }^{17, \dagger}$ J. Xiao, ${ }^{17, \uparrow}$ Z. You, ${ }^{18, \dagger}$ X. Gao, ${ }^{1,, d, \dagger}$ M. Xiao, ${ }^{20, \dagger}$ C. Avila, ${ }^{21, \dagger}$ A. Cabrera, ${ }^{21, \dagger}$ C. Florez, ${ }^{21, \dagger}$ J. Fraga, ${ }^{21, \dagger}$ A. Sarkar, ${ }^{21, \uparrow}$ M. A. Segura Delgado, ${ }^{21, \dagger}$ J. Jaramillo, ${ }^{22, \dagger}$ J. Mejia Guisao, ${ }^{22, \dagger}$ F. Ramirez, ${ }^{22, \dagger}$ J. D. Ruiz Alvarez, ${ }^{22, \dagger}$

C. A. Salazar González, ${ }^{22, \dagger}$ N. Vanegas Arbelaez, ${ }^{22, \dagger}$ D. Giljanovic, ${ }^{23, \dagger}$ N. Godinovic, ${ }^{23, \uparrow}$ D. Lelas, ${ }^{23, \dagger}$ I. Puljak, ${ }^{23, \dagger}$ T. Sculac ${ }^{23, \dagger}$ Z. Antunovic, ${ }^{24, \dagger}$ M. Kovac, ${ }^{24, \dagger}$ V. Brigljevic, ${ }^{25, \dagger}$ D. Ferencek, ${ }^{25, \dagger}$ D. Majumder, ${ }^{25, \dagger}$ B. Mesic, ${ }^{25, \dagger}$ M. Roguljic, ${ }^{2, \dagger}$ A. Starodumov, ${ }^{25, k, \dagger}$ T. Susa ${ }^{25, \dagger}$ M. W. Ather ${ }^{26, \dagger}$ A. Attikis, ${ }^{26, \dagger}$ E. Erodotou, ${ }^{26, \dagger}$ A. Ioannou, ${ }^{26, \dagger}$ G. Kole, ${ }^{26, \uparrow}$ M. Kolosova, ${ }^{26, \dagger}$ S. Konstantinou, ${ }^{26, \dagger}$ G. Mavromanolakis, ${ }^{26, \dagger}$ J. Mousa, ${ }^{26, \dagger}$ C. Nicolaou, ${ }^{26, \dagger}$ F. Ptochos, ${ }^{26, \dagger}$ P. A. Razis, ${ }^{26, \uparrow}$ H. Rykaczewski, ${ }^{26, \dagger}$ H. Saka, ${ }^{26, \dagger}$ D. Tsiakkouri, ${ }^{26, \dagger}$ M. Finger, ${ }^{27,1, \dagger}$ M. Finger Jr. ${ }^{27,1, \dagger}$ A. Kveton, ${ }^{27, \dagger}$ J. Tomsa, ${ }^{27, \dagger}$ E. Ayala,,${ }^{28, \dagger}$ E. Carrera Jarrin, ${ }^{29, \dagger}$ A. A. Abdelalim, ${ }^{30, \mathrm{~m}, \mathrm{n}, \dagger}$ S. Abu Zeid, ${ }^{30,0, \dagger}$ S. Khalil, ${ }^{30, n, \dagger}$ M. A. Mahmoud, ${ }^{31, \dagger}$ Y. Mohammed, ${ }^{31, p, \dagger}$ S. Bhowmik, ${ }^{32, \dagger}$ A. Carvalho Antunes De Oliveira, ${ }^{32, \dagger}$ R. K. Dewanjee, ${ }^{32, \dagger}$ K. Ehataht, ${ }^{32, \dagger}$ M. Kadastik ${ }^{32, \dagger}$ M. Raidal ${ }^{32, \dagger}$ C. Veelken, ${ }^{32, \dagger}$ P. Eerola, ${ }^{33, \uparrow}$ H. Kirschenmann, ${ }^{33, \dagger}$ M. Voutilainen, ${ }^{33, \dagger}$ E. Brücken, ${ }^{34, \dagger}$ J. Havukainen, ${ }^{34, \uparrow}$ V. Karimäki, ${ }^{34, \dagger}$ M. S. Kim, ${ }^{34, \dagger}$ R. Kinnunen, ${ }^{34, \dagger}$ T. Lampén, ${ }^{34, \dagger}$ K. Lassila-Perini, ${ }^{34, \dagger}$ S. Laurila, ${ }^{34, \dagger}$ S. Lehti, ${ }^{34, \dagger}$ T. Lindén, ${ }^{34, \dagger}$ H. Siikonen, ${ }^{34, \dagger}$ E. Tuominen, ${ }^{34, \dagger}$ J. Tuominiemi, ${ }^{34, \dagger}$ P. Luukka, ${ }^{35, \dagger}$ T. Tuuva, ${ }^{35, \dagger}$ M. Besancon, ${ }^{36, \dagger}$ F. Couderc, ${ }^{36, \dagger}$ M. Dejardin, ${ }^{36, \dagger}$ D. Denegri, ${ }^{36, \uparrow}$ J. L. Faure, ${ }^{36, \uparrow}$ F. Ferri, ${ }^{36, \uparrow}$ S. Ganjour, ${ }^{36, \uparrow}$ A. Givernaud, ${ }^{36, \uparrow}$ P. Gras, ${ }^{36, \uparrow}$ G. Hamel de Monchenault, ${ }^{36, \uparrow}$ P. Jarry, ${ }^{36, \dagger}$ B. Lenzi, ${ }^{36, \dagger}$ E. Locci, ${ }^{36, \dagger}$ J. Malcles, ${ }^{36, \dagger}$ J. Rander, ${ }^{36, \dagger}$ A. Rosowsky, ${ }^{36, \dagger}$ M. Ö. Sahin, ${ }^{36, \dagger}$ A. Savoy-Navarro, ${ }^{36, q, \dagger}$ M. Titov, ${ }^{36, \uparrow}$ G. B. Yu, ${ }^{36, \dagger}$ S. Ahuja, ${ }^{37, \dagger}$ C. Amendola, ${ }^{37, \dagger}$ F. Beaudette, ${ }^{37, \dagger}$ M. Bonanomi,${ }^{37, \dagger}$ P. Busson, ${ }^{37, \uparrow}$ C. Charlot,${ }^{37, \dagger}$ O. Davignon, ${ }^{37, \uparrow}$ B. Diab, ${ }^{37, \uparrow}$ G. Falmagne, ${ }^{37, \dagger}$ R. Granier de Cassagnac, ${ }^{37, \uparrow}$ A. Hakimi, ${ }^{37, \dagger}$ I. Kucher, ${ }^{37, \uparrow}$ A. Lobanov, ${ }^{37, \uparrow}$ C. Martin Perez, ${ }^{37, \uparrow}$ M. Nguyen, ${ }^{37, \dagger}$ C. Ochando, ${ }^{37, \dagger}$ P. Paganini, ${ }^{37, \dagger}$ J. Rembser, ${ }^{37, \dagger}$ R. Salerno, ${ }^{37, \dagger}$ J. B. Sauvan, ${ }^{37, \uparrow}$ Y. Sirois,$^{37, \dagger}$ A. Zabi, ${ }^{37, \dagger}$ A. Zghiche, ${ }^{37, \uparrow}$ J.-L. Agram, ${ }^{38, r, \uparrow}$ J. Andrea, ${ }^{38, \uparrow}$ D. Bloch, ${ }^{38, \uparrow}$ G. Bourgatte, ${ }^{38, \dagger}$ J.-M. Brom, ${ }^{38, \uparrow}$ E. C. Chabert, ${ }^{38, \dagger}$ C. Collard, ${ }^{38, \dagger}$ J.-C. Fontaine, ${ }^{38, r, \dagger}$ D. Gelé, ${ }^{38, \dagger}$ U. Goerlach, ${ }^{38, \uparrow}$ C. Grimault, ${ }^{38, \dagger}$ A.-C. Le Bihan, ${ }^{38, \uparrow}$ P. Van Hove, ${ }^{38, \dagger}$ E. Asilar, ${ }^{39, \dagger}$ S. Beauceron, ${ }^{39, \dagger}$ C. Bernet,${ }^{39, \dagger}$ G. Boudoul, ${ }^{39, \dagger}$ C. Camen, ${ }^{39, \dagger}$ A. Carle, ${ }^{39, \dagger}$ N. Chanon, ${ }^{39, \dagger}$ D. Contardo, ${ }^{39, \dagger}$ P. Depasse, ${ }^{39, \dagger}$ H. El Mamouni, ${ }^{39, \dagger}$ J. Fay, ${ }^{39, \dagger}$ S. Gascon, ${ }^{39, \dagger}$ M. Gouzevitch, ${ }^{39, \dagger}$ B. Ille, ${ }^{39, \dagger}$ Sa. Jain, ${ }^{39, \dagger}$ I. B. Laktineh, ${ }^{39, \dagger}$ H. Lattaud, ${ }^{39, \dagger}$ A. Lesauvage, ${ }^{39, \dagger}$ M. Lethuillier, ${ }^{39, \dagger}$ L. Mirabito, ${ }^{39, \dagger}$ L. Torterotot, ${ }^{39, \dagger}$ G. Touquet, ${ }^{39, \dagger}$ M. Vander Donckt, ${ }^{39, \dagger}$ S. Viret, ${ }^{39, \dagger}$ T. Toriashvili, ${ }^{40, s, \dagger}$ Z. Tsamalaidze, ${ }^{40,1, \dagger}$ L. Feld, ${ }^{41, \dagger}$ K. Klein, ${ }^{41, \dagger}$ M. Lipinski, ${ }^{41, \dagger}$ D. Meuser, ${ }^{41, \dagger}$ A. Pauls, ${ }^{41, \dagger}$ M. Preuten,${ }^{41, \dagger}$ M. P. Rauch ${ }^{41, \dagger}$ J. Schulz,${ }^{41, \dagger}$ M. Teroerde, ${ }^{41, \dagger}$ D. Eliseev, ${ }^{42, \dagger}$ M. Erdmann,${ }^{42, \dagger}$ P. Fackeldey, ${ }^{42, \dagger}$ B. Fischer, ${ }^{42, \dagger}$ S. Ghosh ${ }^{42, \dagger}$ T. Hebbeker ${ }^{42, \dagger}$ K. Hoepfner, ${ }^{42, \dagger}$ H. Keller,${ }^{42, \dagger}$ L. Mastrolorenzo, ${ }^{42, \dagger}$ M. Merschmeyer, ${ }^{42, \uparrow}$ A. Meyer, ${ }^{42, \uparrow}$ P. Millet, ${ }^{42, \dagger}$ G. Mocellin,${ }^{42, \dagger}$ S. Mondal, ${ }^{42, \dagger}$ S. Mukherjee, ${ }^{42, \dagger}$ D. Noll, ${ }^{42, \dagger}$ A. Novak, ${ }^{42, \uparrow}$ T. Pook ${ }^{42, \dagger}$ A. Pozdnyakov, ${ }^{42, \dagger}$ T. Quast, ${ }^{42, \dagger}$ M. Radziej, ${ }^{42, \dagger}$ Y. Rath ${ }^{42, \dagger}$ H. Reithler, ${ }^{42, \dagger}$ J. Roemer, ${ }^{42, \dagger}$ A. Schmidt ${ }^{42, \dagger}$ S. C. Schuler, ${ }^{42, \dagger}$ A. Sharma ${ }^{42, \dagger}$ S. Wiedenbeck, ${ }^{42, \dagger}$ S. Zaleski ${ }^{42, \uparrow}$ C. Dziwok, ${ }^{43, \dagger}$ G. Flügge, ${ }^{43, \uparrow}$ W. Haj Ahmad, ${ }^{43, t, \dagger}$ O. Hlushchenko, ${ }^{43, \dagger}$ T. Kress, ${ }^{43, \dagger}$ A. Nowack, ${ }^{43, \uparrow}$ C. Pistone, ${ }^{43, \dagger}$ O. Pooth, ${ }^{43, \uparrow}$ D. Roy, ${ }^{43, \uparrow}$ H. Sert, ${ }^{43, \uparrow}$ A. Stahl, ${ }^{43, u, \uparrow}$ T. Ziemons, ${ }^{43, \dagger}$ H. Aarup Petersen, ${ }^{44, \dagger}$ M. Aldaya Martin, ${ }^{44, \dagger}$ P. Asmuss, ${ }^{44, \dagger}$ I. Babounikau, ${ }^{44, \dagger}$ S. Baxter, ${ }^{44, \dagger}$ O. Behnke ${ }^{44, \dagger}$ A. Bermúdez Martínez, ${ }^{44, \dagger}$ A. A. Bin Anuar, ${ }^{44, \dagger}$ K. Borras,${ }^{4, v, \dagger}$ V. Botta, ${ }^{44, \dagger}$ D. Brunner, ${ }^{44, \uparrow}$ A. Campbell, ${ }^{44, \dagger}$ A. Cardinini, ${ }^{44, \uparrow}$ P. Connor ${ }^{44, \uparrow}$ S. Consuegra Rodríguez,${ }^{44, \dagger}$ V. Danilov, ${ }^{44, \dagger}$ A. De Wit,${ }^{44, \dagger}$ M. M. Defranchis, ${ }^{44, \dagger}$ L. Didukh, ${ }^{44, \uparrow}$

D. Domínguez Damiani ${ }^{44, \dagger}$ G. Eckerlin, ${ }^{44, \dagger}$ D. Eckstein ${ }^{44, \dagger}$ T. Eichhorn, ${ }^{44, \dagger}$ A. Elwood, ${ }^{44, \dagger}$ L. I. Estevez Banos, ${ }^{44, \dagger}$ E. Gallo, ${ }^{44, \mathrm{w}, \dagger}$ A. Geiser, ${ }^{44, \dagger}$ A. Giraldi, ${ }^{44, \dagger}$ A. Grohsjean, ${ }^{44, \uparrow}$ M. Guthoff, ${ }^{44, \dagger}$ A. Harb, ${ }^{44, \uparrow}$ A. Jafari, ${ }^{44, x, \uparrow}$ N. Z. Jomhari, ${ }^{44, \dagger}$ 
H. Jung, ${ }^{44, \dagger}$ A. Kasem, ${ }^{44, v, \dagger}$ M. Kasemann, ${ }^{44, \uparrow}$ H. Kaveh, ${ }^{44, \uparrow}$ C. Kleinwort, ${ }^{44, \dagger}$ J. Knolle, ${ }^{44, \dagger}$ D. Krücker, ${ }^{44, \uparrow}$ W. Lange ${ }^{44, \uparrow}$ T. Lenz, ${ }^{44, \dagger}$ J. Lidrych, ${ }^{44, \dagger}$ K. Lipka, ${ }^{44, \dagger}$ W. Lohmann, ${ }^{44, y, \dagger}$ R. Mankel, ${ }^{44, \dagger}$ I.-A. Melzer-Pellmann, ${ }^{44, \dagger}$ J. Metwally, ${ }^{44, \dagger}$ A. B. Meyer, ${ }^{4, \dagger}$ M. Meyer, ${ }^{44, \dagger}$ M. Missiroli, ${ }^{44, \dagger}$ J. Mnich ${ }^{44, \uparrow}$ A. Mussgiller, ${ }^{44, \dagger}$ V. Myronenko, ${ }^{44, \dagger}$ Y. Otarid, ${ }^{44, \dagger}$ D. Pérez Adán, ${ }^{44, \dagger}$ S. K. Pflitsch, ${ }^{44, \dagger}$ D. Pitzl, ${ }^{44, \dagger}$ A. Raspereza, ${ }^{44, \dagger}$ A. Saggio,${ }^{44, \dagger}$ A. Saibel, ${ }^{44, \dagger}$ M. Savitskyi, ${ }^{44, \dagger}$ V. Scheurer, ${ }^{44, \dagger}$ P. Schütze, ${ }^{44, \dagger}$ C. Schwanenberger ${ }^{44, \uparrow}$ R. Shevchenko, ${ }^{44, \dagger}$ A. Singh, ${ }^{44, \uparrow}$ R. E. Sosa Ricardo, ${ }^{44, \dagger}$ H. Tholen, ${ }^{44, \uparrow}$ N. Tonon, ${ }^{44, \dagger}$ O. Turkot, ${ }^{44, \dagger}$ A. Vagnerini, ${ }^{44, \dagger}$ M. Van De Klundert, ${ }^{44, \dagger}$ R. Walsh, ${ }^{44, \dagger}$ D. Walter, ${ }^{44, \dagger}$ Y. Wen, ${ }^{44, \dagger}$ K. Wichmann,${ }^{44, \dagger}$ C. Wissing, ${ }^{44, \dagger}$ S. Wuchterl, ${ }^{44, \dagger}$ O. Zenaiev, ${ }^{44, \dagger}$ R. Zlebcik ${ }^{44, \dagger}$ R. Aggleton, ${ }^{45, \dagger}$ S. Bein, ${ }^{45, \dagger}$ L. Benato, ${ }^{45, \uparrow}$ A. Benecke, ${ }^{45, \dagger}$ K. De Leo, ${ }^{45, \dagger}$ T. Dreyer, ${ }^{45, \dagger}$ A. Ebrahimi,${ }^{45, \dagger}$ F. Feindt,${ }^{45, \dagger}$ A. Fröhlich, ${ }^{45, \dagger}$ C. Garbers, ${ }^{45, \dagger}$ E. Garutti, ${ }^{45, \uparrow}$ P. Gunnellini, ${ }^{45, \dagger}$ J. Haller, ${ }^{45, \dagger}$ A. Hinzmann, ${ }^{45, \dagger}$ A. Karavdina,${ }^{45, \dagger}$ G. Kasieczka, ${ }^{45, \dagger}$ R. Klanner, ${ }^{45, \dagger}$ R. Kogler, ${ }^{45, \dagger}$ V. Kutzner, ${ }^{45, \dagger}$ J. Lange, ${ }^{45, \dagger}$ T. Lange, ${ }^{45, \dagger}$ A. Malara, ${ }^{45,}$ J. Multhaup, ${ }^{45, \dagger}$ C. E. N. Niemeyer, ${ }^{45, \dagger}$ A. Nigamova, ${ }^{45, \dagger}$ K. J. Pena Rodriguez, ${ }^{45, \dagger}$ O. Rieger, ${ }^{45, \dagger}$ P. Schleper, ${ }^{45, \dagger}$ S. Schumann, ${ }^{45, \dagger}$ J. Schwandt, ${ }^{45, \dagger}$ D. Schwarz, ${ }^{45, \dagger}$ J. Sonneveld ${ }^{45, \uparrow}$ H. Stadie, ${ }^{45, \dagger}$ G. Steinbrück, ${ }^{45, \dagger}$ B. Vormwald, ${ }^{45, \dagger}$ I. Zoi, ${ }^{45, \dagger}$ M. Baselga, ${ }^{46, \dagger}$ S. Baur, ${ }^{46, \dagger}$ J. Bechtel, ${ }^{46, \dagger}$ T. Berger, ${ }^{46, \dagger}$ E. Butz, ${ }^{46, \dagger}$ R. Caspart, ${ }^{46, \dagger}$ T. Chwalek, ${ }^{46, \dagger}$ W. De Boer ${ }^{46, \uparrow}$ A. Dierlamm, ${ }^{46, \dagger}$ A. Droll, ${ }^{46, \dagger}$ K. El Morabit, ${ }^{46, \uparrow}$

N. Faltermann, ${ }^{46, \dagger}$ K. Flöh, ${ }^{46, \dagger}$ M. Giffels, ${ }^{46, \uparrow}$ A. Gottmann, ${ }^{46, \dagger}$ F. Hartmann, ${ }^{46, u, \uparrow}$ C. Heidecker, ${ }^{46, \uparrow}$ U. Husemann, ${ }^{46, \uparrow}$ M. A. Iqbal,${ }^{46, \dagger}$ I. Katkov, ${ }^{46, z, \dagger}$ P. Keicher ${ }^{46, \dagger}$ R. Koppenhöfer, ${ }^{46, \uparrow}$ S. Maier, ${ }^{46, \dagger}$ M. Metzler, ${ }^{46, \dagger}$ S. Mitra, ${ }^{46, \dagger}$ M. U. Mozer, ${ }^{46, \dagger}$ D. Müller, ${ }^{46, \dagger}$ Th. Müller, ${ }^{46, \dagger}$ M. Musich, ${ }^{46, \dagger}$ G. Quast, ${ }^{46, \dagger}$ K. Rabbertz, ${ }^{46, \dagger}$ J. Rauser, ${ }^{46, \dagger}$ D. Savoiu, ${ }^{46, \uparrow}$ D. Schäfer, ${ }^{46, \uparrow}$ M. Schnepf, ${ }^{46, \uparrow}$ M. Schröder, ${ }^{46, \uparrow}$ D. Seith, ${ }^{46, \uparrow}$ I. Shvetsov, ${ }^{46, \dagger}$ H. J. Simonis, ${ }^{46, \dagger}$ R. Ulrich, ${ }^{46, \dagger}$ M. Wassmer,${ }^{46, \dagger}$ M. Weber, ${ }^{46, \uparrow}$ C. Wöhrmann ${ }^{46, \dagger}$ R. Wolf, ${ }^{46, \dagger}$ S. Wozniewski, ${ }^{46, \uparrow}$ G. Anagnostou, ${ }^{47, \dagger}$ P. Asenov, ${ }^{47, \dagger}$ G. Daskalakis, ${ }^{47, \dagger}$ T. Geralis, ${ }^{47, \dagger}$ A. Kyriakis, ${ }^{47, \dagger}$ D. Loukas, ${ }^{47, \dagger}$ G. Paspalaki, ${ }^{47, \uparrow}$ A. Stakia, ${ }^{47, \dagger}$ M. Diamantopoulou, ${ }^{48, \uparrow}$ D. Karasavvas, ${ }^{48, \uparrow}$ G. Karathanasis, ${ }^{48, \uparrow}$ P. Kontaxakis, ${ }^{48, \dagger}$ C. K. Koraka, ${ }^{48, \dagger}$ A. Manousakis-katsikakis, ${ }^{48, \dagger}$ A. Panagiotou, ${ }^{48, \dagger}$ I. Papavergou, ${ }^{48, \dagger}$ N. Saoulidou, ${ }^{48, \uparrow}$ K. Theofilatos, ${ }^{48, \dagger}$ K. Vellidis, ${ }^{48, \dagger}$ E. Vourliotis ${ }^{48, \uparrow}$ G. Bakas, ${ }^{49, \dagger}$ K. Kousouris, ${ }^{49, \dagger}$ I. Papakrivopoulos, ${ }^{49, \dagger}$ G. Tsipolitis,${ }^{49, \uparrow}$ A. Zacharopoulou, ${ }^{49, \dagger}$ I. Evangelou, ${ }^{50, \uparrow}$ C. Foudas, ${ }^{50, \dagger}$ P. Gianneios, ${ }^{50, \dagger}$ P. Katsoulis, ${ }^{50, \dagger}$ P. Kokkas, ${ }^{50, \dagger}$ S. Mallios, ${ }^{50, \uparrow}$ K. Manitara, ${ }^{50, \dagger}$ N. Manthos, ${ }^{50, \dagger}$ I. Papadopoulos,${ }^{50, \dagger}$ J. Strologas,${ }^{50, \dagger}$ M. Bartók,${ }^{51, a a, \dagger}$ R. Chudasama,${ }^{51, \dagger}$ M. M. A. Gadallah, ${ }^{51, b b, \dagger}$ S. Lökös,${ }^{51, c c, \dagger}$ P. Major,${ }^{51, \dagger}$ K. Mandal, ${ }^{51, \uparrow}$ A. Mehta, ${ }^{51, \dagger}$ G. Pasztor,,${ }^{51, \dagger}$ O. Surányi, ${ }^{51, \dagger}$ G. I. Veres, ${ }^{51, \dagger}$ G. Bencze,${ }^{52, \dagger}$ C. Hajdu, ${ }^{52, \dagger}$ D. Horvath, ${ }^{52, \mathrm{dd}, \dagger}$ F. Sikler, ${ }^{52, \dagger}$ V. Veszpremi, ${ }^{52, \dagger}$ G. Vesztergombi, ${ }^{52, \mathrm{a}, \mathrm{cc}, \uparrow}$ S. Czellar, ${ }^{53, \uparrow}$ J. Karancsi ${ }^{53, a a, \uparrow}$ J. Molnar, ${ }^{53, \dagger}$ Z. Szillasi, ${ }^{53, \dagger}$ D. Teyssier, ${ }^{53, \dagger}$ P. Raics, ${ }^{54, \uparrow}$ Z. L. Trocsanyi,${ }^{54, \dagger}$ B. Ujvari, ${ }^{54, \uparrow}$ S. Choudhury, ${ }^{56, \dagger}$ J. R. Komaragiri, ${ }^{56, \dagger}$ D. Kumar ${ }^{56, \dagger}$ L. Panwar, ${ }^{56, \dagger}$ P. C. Tiwari, ${ }^{56, \dagger}$ S. Bahinipati, ${ }^{57, e,, \dagger}$ D. Dash, ${ }^{57, \dagger}$ C. Kar, ${ }^{57, \dagger}$ P. Mal, ${ }^{57, \dagger}$ T. Mishra, ${ }^{57, \dagger}$ V. K. Muraleedharan Nair Bindhu, ${ }^{57, \dagger}$ A. Nayak, ${ }^{57, f f, \dagger}$ D. K. Sahoo, ${ }^{57, e e, \dagger}$ N. Sur, ${ }^{57, \dagger}$ S. K. Swain, ${ }^{57, \uparrow}$ S. Bansal, ${ }^{58, \uparrow}$ S. B. Beri, ${ }^{58, \uparrow}$ V. Bhatnagar, ${ }^{58, \uparrow}$ S. Chauhan, ${ }^{58, \uparrow}$ N. Dhingra, ${ }^{58, g g, \dagger}$ R. Gupta, ${ }^{58, \dagger}$ A. Kaur, ${ }^{58, \uparrow}$ S. Kaur, ${ }^{58, \uparrow}$ P. Kumari, ${ }^{58, \uparrow}$ M. Lohan, ${ }^{58, \dagger}$ M. Meena ${ }^{58, \dagger}$ K. Sandeep, ${ }^{58, \dagger}$ S. Sharma ${ }^{58, \dagger}$ J. B. Singh, ${ }^{58, \dagger}$ A. K. Virdi, ${ }^{58, \uparrow}$ A. Ahmed ${ }^{59, \dagger}$ A. Bhardwaj, ${ }^{59, \dagger}$ B. C. Choudhary, ${ }^{59, \dagger}$ R. B. Garg, ${ }^{59, \dagger}$ M. Gola, ${ }^{5, \dagger}$ S. Keshri, ${ }^{59, \dagger}$ A. Kumar, ${ }^{59, \dagger}$ M. Naimuddin, ${ }^{59, \dagger}$ P. Priyanka,${ }^{59, \dagger}$ K. Ranjan, ${ }^{59, \dagger}$ A. Shah, ${ }^{59, \dagger}$ M. Bharti,${ }^{60, h h, \dagger}$ R. Bhattacharya,${ }^{60, \uparrow}$ S. Bhattacharya ${ }^{60, \uparrow}$ D. Bhowmik, ${ }^{60, \dagger}$ S. Dutta, ${ }^{60, \uparrow}$ S. Ghosh ${ }^{60, \dagger}$ B. Gomber,${ }^{60, i i, \uparrow}$ M. Maity, ${ }^{60, j j, \dagger}$ S. Nandan, ${ }^{60, \uparrow}$ P. Palit, ${ }^{60, \uparrow}$ A. Purohit, ${ }^{60, \uparrow}$ P. K. Rout, ${ }^{60, \uparrow}$ G. Saha,${ }^{60, \uparrow}$ S. Sarkar, ${ }^{60, \dagger}$ M. Sharan,${ }^{60, \dagger}$ B. Singh, ${ }^{60, h h, \dagger}$ S. Thakur, ${ }^{60, h h, \dagger}$ P. K. Behera, ${ }^{61, \uparrow}$ S. C. Behera, ${ }^{61, \uparrow}$ P. Kalbhor ${ }^{61, \dagger}$ A. Muhammad,${ }^{61, \dagger}$ R. Pradhan,${ }^{61, \dagger}$ P. R. Pujahari,${ }^{61, \dagger}$ A. Sharma,${ }^{61, \dagger}$ A. K. Sikdar, ${ }^{61, \dagger}$ D. Dutta, ${ }^{62, \dagger}$ V. Jha, ${ }^{62, \dagger}$ V. Kumar, ${ }^{6, \dagger}$ D. K. Mishra, ${ }^{62, \dagger}$ K. Naskar, ${ }^{62, k k, \dagger}$ P. K. Netrakanti, ${ }^{62, \dagger}$ L. M. Pant, ${ }^{62, \dagger}$ P. Shukla, ${ }^{62, \dagger}$ T. Aziz, ${ }^{6, \dagger}$ M. A. Bhat,${ }^{63, \dagger}$ S. Dugad, ${ }^{63, \dagger}$ R. Kumar Verma, ${ }^{63, \dagger}$ U. Sarkar, ${ }^{63, \dagger}$ S. Banerjee, ${ }^{64, \dagger}$ S. Bhattacharya,${ }^{64, \dagger}$ S. Chatterjee, ${ }^{64, \dagger}$ P. Das, ${ }^{64, \uparrow}$ M. Guchait, ${ }^{64, \dagger}$ S. Karmakar, ${ }^{64, \dagger}$ S. Kumar, ${ }^{64, \dagger}$ G. Majumder ${ }^{64, \dagger}$ K. Mazumdar, ${ }^{64, \dagger}$ S. Mukherjee, ${ }^{64, \dagger}$ D. Roy, ${ }^{64, \dagger}$ N. Sahoo, ${ }^{64, \dagger}$

S. Dube, ${ }^{65, \dagger}$ B. Kansal, ${ }^{6, \dagger}$ A. Kapoor,${ }^{65, \dagger}$ K. Kothekar, ${ }^{65, \dagger}$ S. Pandey, ${ }^{65, \dagger}$ A. Rane, ${ }^{65, \dagger}$ A. Rastogi, ${ }^{65, \dagger}$ S. Sharma ${ }^{65, \dagger}$

H. Bakhshiansohi, ${ }^{66,11, \dagger}$ S. Chenarani, ${ }^{67, \mathrm{~mm}, \dagger}$ S. M. Etesami ${ }^{67, \dagger}$ M. Khakzad,${ }^{67, \dagger}$ M. Mohammadi Najafabadi, ${ }^{67, \dagger}$

M. Felcini ${ }^{68, \dagger}$ M. Grunewald, ${ }^{68, \dagger}$ M. Abbrescia,${ }^{69 a, 69 b, \dagger}$ R. Aly, ${ }^{69,69 b, n n, \dagger}$ C. Aruta, ${ }^{69 a, 69 b, \dagger}$ A. Colaleo, ${ }^{69 a, \dagger}$ D. Creanza, ${ }^{69 a, 69 c, \uparrow}$

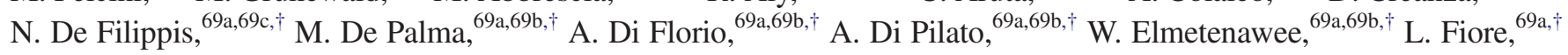
A. Gelmi ${ }^{69 a, 69 b, \dagger}$ M. Gul ${ }^{69 a, \dagger}$ G. Iaselli, ${ }^{69 a, 69 c, \dagger}$ M. Ince,${ }^{69 a, 69 b, \dagger}$ S. Lezki,${ }^{69 a, 69 b, \dagger}$ G. Maggi, ${ }^{69 a, 69 c, \dagger}$ M. Maggi, ${ }^{69 a, \dagger}$ I. Margjeka ${ }^{69 a, 69 b, \dagger}$ J. A. Merlin, ${ }^{69 a, \dagger}$ S. My ${ }^{69 a, 69 b, \dagger}$ S. Nuzzo, ${ }^{69 a, 69 b, \dagger}$ A. Pompili, ${ }^{69 a, 69 b, \uparrow}$ G. Pugliese, ${ }^{69 a, 69 c, \dagger}$ A. Ranieri, ${ }^{69 a, \dagger}$ G. Selvaggi ${ }^{69 \mathrm{a}, 69 \mathrm{~b}, \dagger}$ L. Silvestris ${ }^{69 \mathrm{a}, \dagger}$ F. M. Simone,${ }^{69 \mathrm{a}, 69 \mathrm{~b}, \dagger}$ R. Venditti, ${ }^{69 \mathrm{a}, \dagger}$ P. Verwilligen ${ }^{69 \mathrm{a}, \uparrow}$ G. Abbiendi, ${ }^{70 \mathrm{a}, \uparrow}$ C. Battilana ${ }^{70 a, 70 b, \dagger}$ D. Bonacorsi,${ }^{70 a, 70 b, \dagger}$ L. Borgonovi, ${ }^{70 a, 70 b, \dagger}$ S. Braibant-Giacomelli, ${ }^{70 a, 70 b, \dagger}$ R. Campanini, ${ }^{70 a, 70 b, \dagger}$ P. Capiluppi, ${ }^{70 a, 70 b, \dagger}$ A. Castro ${ }^{70 a, 70 b, \dagger}$ F. R. Cavallo, ${ }^{70 a, \dagger}$ C. Ciocca,${ }^{70 a, \dagger}$ M. Cuffiani,${ }^{70 a, 70 b, \dagger}$ G. M. Dallavalle, ${ }^{70 a, \dagger}$ T. Diotalevi, ${ }^{70 a, 70 b, \dagger}$ F. Fabbri, ${ }^{70 a, \dagger}$ A. Fanfani,${ }^{70 a, 70 b, \dagger}$ E. Fontanesi, ${ }^{70 a, 70 b, \dagger}$ P. Giacomelli, ${ }^{70 a, \dagger}$ L. Giommi, ${ }^{70 a, 70 b, \uparrow}$ C. Grandi, ${ }^{70 a, \dagger}$ L. Guiducci, ${ }^{70 a, 70 b, \dagger}$ F. Iemmi, ${ }^{70 a, 70 b, \dagger}$ S. Lo Meo, ${ }^{70 a, 00, \dagger}$ S. Marcellini, ${ }^{70 a, \dagger}$ G. Masetti, ${ }^{70 a, \dagger}$ 
F. L. Navarria, ${ }^{70 a, 70 b, \dagger}$ A. Perrotta,$^{70 a, \dagger}$ F. Primavera, ${ }^{70 a, 70 b, \dagger}$ T. Rovelli ${ }^{70 a, 70 b, \dagger}$ G. P. Siroli,${ }^{70 a, 70 b, \dagger}$ N. Tosi,${ }^{70 a, \dagger}$ S. Albergo, ${ }^{71 a, 71 b, p p, \dagger}$ S. Costa, ${ }^{71 a, 71 b, \dagger}$ A. Di Mattia, ${ }^{71 a, \dagger}$ R. Potenza, ${ }^{71 a, 71 b, \dagger}$ A. Tricomi,${ }^{71 a, 71 b, p p, \dagger}$ C. Tuve, ${ }^{71 a, 71 b, \dagger}$ G. Barbagli ${ }^{72 a,{ }^{\dagger}}$ A. Cassese,${ }^{72 a, \dagger}$ R. Ceccarelli, ${ }^{72 a, 72 b, \dagger}$ V. Ciulli,${ }^{72 a, 72 b, \dagger}$ C. Civinini,${ }^{72 a, \dagger}$ R. D’Alessandro, ${ }^{72 a, 72 b, \dagger}$ F. Fiori, ${ }^{72 a, \dagger}$ E. Focardi ${ }^{72 a, 72 b, \dagger}$ G. Latino, ${ }^{72 a, 72 b, \dagger}$ P. Lenzi, ${ }^{72 a, 72 b, \dagger}$ M. Lizzo, ${ }^{72 a, 72 b, \dagger}$ M. Meschini,${ }^{72 a,{ }^{\dagger}}$ S. Paoletti, ${ }^{72 a,{ }^{\dagger}}$ R. Seidita, ${ }^{72 a, 72 b, \dagger}$ G. Sguazzoni, ${ }^{72 a, \dagger}$ L. Viliani, ${ }^{72 a, \uparrow}$ L. Benussi, ${ }^{73, \dagger}$ S. Bianco, ${ }^{73, \dagger}$ D. Piccolo, ${ }^{73, \dagger}$ F. Ferro, ${ }^{74 a, \uparrow}$ R. Mulargia,${ }^{74 a, 74 b, \dagger}$ E. Robutti ${ }^{74 a, \uparrow}$ S. Tosi ${ }^{74 a, 74 b, \dagger}$ A. Benaglia, ${ }^{75 a,{ }^{\dagger}}$ A. Beschi,${ }^{75 a, 75 b, \dagger}$ F. Brivio,${ }^{75 a, 75 b, \dagger}$ F. Cetorelli, ${ }^{75 a, 75 b, \dagger}$ V. Ciriolo, ${ }^{75 a, 75 b, u, ~}$, F. De Guio, ${ }^{75 a, 75 b, \dagger}$ M. E. Dinardo,${ }^{75 a, 75 b, \dagger}$ P. Dini ${ }^{75 a, \uparrow}$ S. Gennai,${ }^{75 a, \dagger}$ A. Ghezzi, ${ }^{75 a, 75 b, \dagger}$ P. Govoni, ${ }^{75 a, 75 b, \dagger}$ L. Guzzi,${ }^{75 a, 75 b, \dagger}$ M. Malberti, ${ }^{75 a, \dagger}$ S. Malvezzi ${ }^{75 a, \dagger}$ D. Menasce,${ }^{75 a, \dagger}$ F. Monti, ${ }^{75 a, 75 b, \dagger}$ L. Moroni, ${ }^{75 a, \dagger}$ M. Paganoni, ${ }^{75 a, 75 b, \dagger}$ D. Pedrini,${ }^{75 a, \uparrow}$ S. Ragazzi ${ }^{75 a, 75 b, \dagger}$ T. Tabarelli de Fatis, ${ }^{75 a, 75 b, \dagger}$ D. Valsecchi ${ }^{75 a, 75 b, u, \dagger}$ D. Zuolo, ${ }^{75 a, 75 b, \dagger}$ S. Buontempo, ${ }^{76 a, \dagger}$ N. Cavallo, ${ }^{76 a, 76 c, \uparrow}$ A. De Iorio, ${ }^{76 a, 76 b, \dagger}$ F. Fabozzi, ${ }^{76 a, 76 c, \dagger}$ F. Fienga, ${ }^{76 a,{ }^{\dagger}}$ A. O. M. Iorio, ${ }^{76 a, 76 b, \dagger}$ L. Layer, ${ }^{76 a, 76 b, \dagger}$ L. Lista, ${ }^{76 a, 76 b,{ }^{\dagger}}$

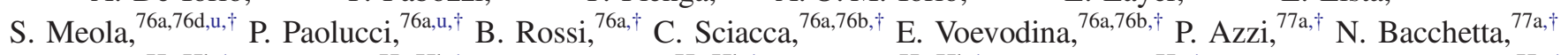
D. Bisello, ${ }^{77 a, 77 b, \uparrow}$ A. Boletti ${ }^{77 a, 77 b, \dagger}$ A. Bragagnolo, ${ }^{77 a, 77 b, \dagger}$ R. Carlin,${ }^{77 a, 77 b, \dagger}$ P. Checchia, ${ }^{77 a, \dagger}$ P. De Castro Manzano, ${ }^{77 a, \uparrow}$ T. Dorigo, ${ }^{77 a, \dagger}$ F. Gasparini, ${ }^{77 a, 77 b, \dagger}$ U. Gasparini, ${ }^{77 a, 77 b,{ }^{\dagger}}$ S. Y. Hoh, ${ }^{77 a, 77 b, \dagger}$ M. Margoni, ${ }^{77 a, 77 b, \dagger}$ A. T. Meneguzzo, ${ }^{77 a, 77 b, \dagger}$ M. Presilla, ${ }^{77 a, 77 b, \dagger}$ P. Ronchese,${ }^{77 a, 77 b, \dagger}$ R. Rossin, ${ }^{77 a, 77 b, \dagger}$ F. Simonetto, ${ }^{77 a, 77 b, \dagger}$ G. Strong, ${ }^{77 a, \uparrow}$ A. Tiko, ${ }^{77 a, \uparrow}$ M. Tosi, ${ }^{77 a, 77 b, \dagger}$ M. Zanetti, ${ }^{77 a, 77 b, \dagger}$ P. Zotto, ${ }^{77 a, 77 b, \dagger}$ A. Zucchetta,${ }^{77 a, 77 b, \dagger}$ G. Zumerle, ${ }^{77 a, 77 b, \dagger}$ A. Braghieri, ${ }^{78 a, \dagger}$ S. Calzaferri, ${ }^{78 a, 78 b, \dagger}$

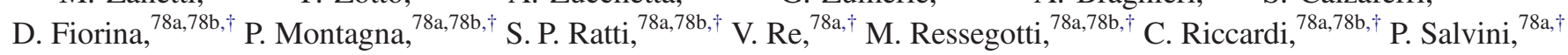
I. Vai, ${ }^{78 a,{ }^{\dagger}}$ P. Vitulo, ${ }^{78 a, 78 b, \dagger}$ M. Biasini,${ }^{79 a, 79 b, \dagger}$ G. M. Bilei ${ }^{79 a, \dagger}$ D. Ciangottini, ${ }^{79 a, 79 b, \dagger}$ L. Fanò ${ }^{79 a, 79 b, \dagger}$ P. Lariccia, ${ }^{79 a, 79 b, \dagger}$ G. Mantovani, ${ }^{79 a, 79 b, \dagger}$ V. Mariani, ${ }^{79 a, 79 b, \dagger}$ M. Menichelli, ${ }^{79 a, \dagger}$ F. Moscatelli, ${ }^{79 a, \uparrow}$ A. Rossi, ${ }^{79 a, 79 b, \dagger}$ A. Santocchia, ${ }^{79 a, 79 b, \dagger}$ D. Spiga, ${ }^{79 a, \dagger}$ T. Tedeschi, ${ }^{79 a, 79 b,{ }^{\dagger}}$ K. Androsov,${ }^{80 a, \dagger}$ P. Azzurri, ${ }^{80 a,{ }^{\dagger}}$ G. Bagliesi, ${ }^{80 a,{ }^{\dagger}}$ V. Bertacchi, ${ }^{80 a, 80 c, \dagger}$ L. Bianchini, ${ }^{80 a,{ }^{\dagger}}$

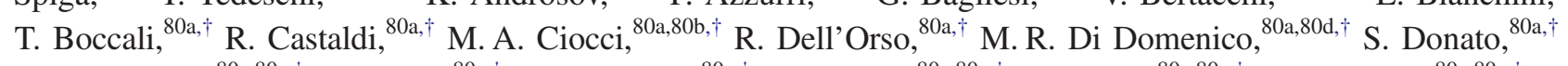

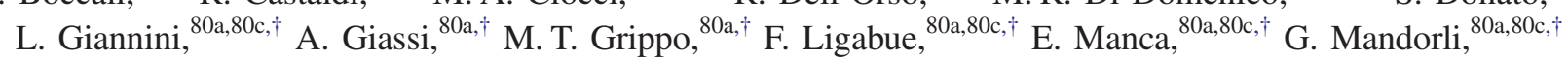

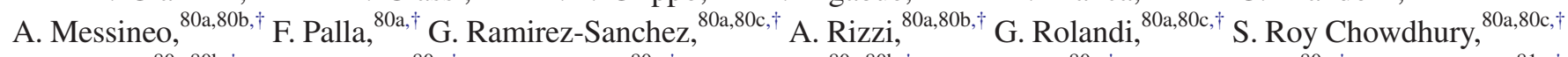
N. Shafiei ${ }^{80 a, 80 b, \dagger}$ P. Spagnolo, ${ }^{80 a, \dagger}$ R. Tenchini ${ }^{80 a, \uparrow}$ G. Tonelli,${ }^{80 a, 80 b, \dagger}$ A. Venturi, ${ }^{80 a, \dagger}$ P. G. Verdini,${ }^{80 a, \dagger}$ F. Cavallari, ${ }^{81 a, \uparrow}$ M. Cipriani,${ }^{81 \mathrm{a}, 81 \mathrm{~b}, \uparrow}$ D. Del Re,${ }^{81 \mathrm{a}, 81 \mathrm{~b}, \uparrow}$ E. Di Marco,${ }^{81 \mathrm{a}, \dagger}$ M. Diemoz,${ }^{81 \mathrm{a}, \uparrow}$ E. Longo,${ }^{81 \mathrm{a}, 81 \mathrm{~b}, \uparrow}$ P. Meridiani, ${ }^{81 \mathrm{a}, \uparrow}$

G. Organtini ${ }^{81 a, 81 b, \dagger}$ F. Pandolfi, ${ }^{81 a, \dagger}$ R. Paramatti, ${ }^{81 a, 81 b, \dagger}$ C. Quaranta, ${ }^{81 a, 81 b, \dagger}$ S. Rahatlou, ${ }^{81 a, 81 b, \dagger}$ C. Rovelli, ${ }^{81 a, \dagger}$

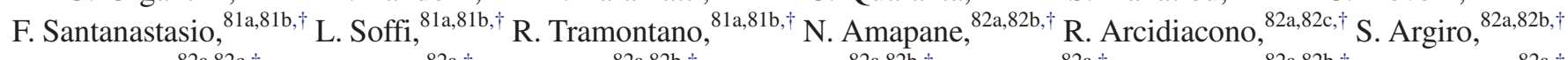

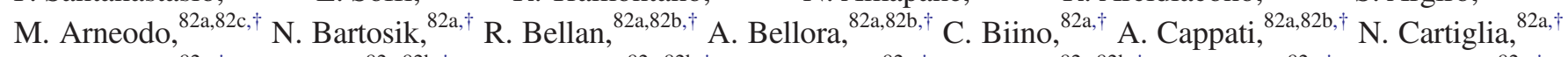
S. Cometti, ${ }^{82 a, \dagger}$ M. Costa, ${ }^{82 a, 82 b, \dagger}$ R. Covarelli, ${ }^{82 a, 82 b, \dagger}$ N. Demaria, ${ }^{82 a, \dagger}$ B. Kiani, ${ }^{82 a, 82 b, \dagger}$ F. Legger, ${ }^{82 a, \uparrow}$ C. Mariotti ${ }^{82 a, \uparrow}$ S. Maselli, ${ }^{82 a, \uparrow}$ E. Migliore, ${ }^{82 a, 82 b, \dagger}$ V. Monaco, ${ }^{82 a, 82 b, \dagger}$ E. Monteil,${ }^{82 a, 82 b, \dagger}$ M. Monteno, ${ }^{82 a, \dagger}$ M. M. Obertino, ${ }^{82 a, 82 b, \dagger}$ G. Ortona, ${ }^{82 a, \dagger}$ L. Pacher, ${ }^{82 a, 82 b, \dagger}$ N. Pastrone,${ }^{82 a, \dagger}$ M. Pelliccioni,${ }^{82 a, \dagger}$ G. L. Pinna Angioni,${ }^{82 a, 82 b, \dagger}$ M. Ruspa,${ }^{82 a, 82 c, \dagger}$ R. Salvatico, ${ }^{82 a, 82 \mathrm{~b}, \dagger}$ F. Siviero, ${ }^{82 \mathrm{a}, 82 \mathrm{~b}, \dagger}$ V. Sola,${ }^{82 \mathrm{a}, \uparrow}$ A. Solano, ${ }^{82 \mathrm{a}, 82 \mathrm{~b}, \dagger}$ D. Soldi,${ }^{82 \mathrm{a}, 82 \mathrm{~b}, \dagger}$ A. Staiano, ${ }^{82 \mathrm{a}, \uparrow}$ D. Trocino, ${ }^{82 \mathrm{a}, 82 \mathrm{~b}, \uparrow}$ S. Belforte,${ }^{83 a, \uparrow}$ V. Candelise,${ }^{83 a, 83 b, \dagger}$ M. Casarsa, ${ }^{83 a, \dagger}$ F. Cossutti ${ }^{83 a, \uparrow}$ A. Da Rold,${ }^{83 a, 83 b, \dagger}$ G. Della Ricca, ${ }^{83 a, 83 b, \dagger}$ F. Vazzoler, ${ }^{83 a, 83 b, \dagger}$ S. Dogra,${ }^{84, \dagger}$ C. Huh,${ }^{84, \uparrow}$ B. Kim,${ }^{84, \uparrow}$ D. H. Kim,${ }^{84, \uparrow}$ G. N. Kim,${ }^{84, \dagger}$ J. Lee, ${ }^{84, \uparrow}$ S. W. Lee,${ }^{84, \uparrow}$ C. S. Moon, ${ }^{84, \uparrow}$ Y. D. Oh,${ }^{84, \dagger}$ S. I. Pak, ${ }^{84, \dagger}$ S. Sekmen,${ }^{84, \dagger}$ Y. C. Yang, ${ }^{84, \dagger}$ H. Kim,${ }^{85, \dagger}$ D. H. Moon, ${ }^{85,}$ B. Francois, ${ }^{86, \dagger}$ T. J. Kim, ${ }^{86, \dagger}$ J. Park, ${ }^{86, \uparrow}$ S. Cho,${ }^{87, \dagger}$ S. Choi,${ }^{87, \dagger}$ Y. Go ${ }^{87, \uparrow}$ S. Ha,${ }^{87, \dagger}$ B. Hong,${ }^{87, \dagger}$ K. Lee,${ }^{87, \dagger}$ K. S. Lee,${ }^{87, \dagger}$ J. Lim, ${ }^{87, \dagger}$ J. Park, ${ }^{87, \dagger}$ S. K. Park, ${ }^{87, \uparrow}$ J. Yoo, ${ }^{87, \dagger}$ J. Goh,${ }^{88, \dagger}$ A. Gurtu, ${ }^{88, \dagger}$ H. S. Kim,${ }^{89, \dagger}$ Y. Kim,${ }^{89, \dagger}$ J. Almond ${ }^{90, \dagger}$ J. H. Bhyun, ${ }^{90, \dagger}$ J. Choi, ${ }^{90, \dagger}$ S. Jeon, ${ }^{90, \dagger}$ J. Kim, ${ }^{90, \uparrow}$ J. S. Kim,${ }^{90, \dagger}$ S. Ko, ${ }^{90, \dagger}$ H. Kwon, ${ }^{90, \dagger}$ H. Lee, ${ }^{90, \dagger}$ K. Lee, ${ }^{90, \dagger}$ S. Lee, ${ }^{90, \uparrow}$ K. Nam, ${ }^{90, \dagger}$ B. H. Oh, ${ }^{90, \dagger}$ M. Oh, ${ }^{90, \dagger}$ S. B. Oh, ${ }^{90, \uparrow}$ B. C. Radburn-Smith, ${ }^{90, \dagger}$ H. Seo,${ }^{90, \dagger}$ U. K. Yang, ${ }^{90, \dagger}$ I. Yoon,${ }^{90, \dagger}$ D. Jeon, ${ }^{91, \dagger}$ J. H. Kim, ${ }^{91, \dagger}$ B. Ko, ${ }^{91, \dagger}$ J. S. H. Lee, ${ }^{91, \dagger}$ I. C. Park,${ }^{91, \dagger}$ Y. Roh,${ }^{91, \dagger}$ D. Song,${ }^{91, \dagger}$ I. J. Watson, ${ }^{91, \dagger}$ H. D. Yoo, ${ }^{92, \dagger}$ Y. Choi, ${ }^{93, \dagger}$ C. Hwang, ${ }^{93, \dagger}$ Y. Jeong, ${ }^{93, \dagger}$ H. Lee, ${ }^{93, \dagger}$ Y. Lee, ${ }^{93, \dagger}$ I. Yu,${ }^{93, \dagger}$ Y. Maghrbi, ${ }^{94, \dagger}$ V. Veckalns, ${ }^{95, q q, \dagger}$ A. Juodagalvis, ${ }^{96, \dagger}$ A. Rinkevicius, ${ }^{96, \dagger}$ G. Tamulaitis, ${ }^{96, \dagger}$ W. A. T. Wan Abdullah,,${ }^{97, \dagger}$ M. N. Yusli, ${ }^{97, \dagger}$ Z. Zolkapli, ${ }^{97, \dagger}$ J. F. Benitez, ${ }^{98, \dagger}$ A. Castaneda Hernandez, ${ }^{98, \uparrow}$ J. A. Murillo Quijada, ${ }^{98, \dagger}$ L. Valencia Palomo, ${ }^{98, \dagger}$ H. Castilla-Valdez,${ }^{99, \dagger}$ E. De La Cruz-Burelo, ${ }^{99, \dagger}$

I. Heredia-De La Cruz, ${ }^{99, r, \dagger}$ R. Lopez-Fernandez, ${ }^{99, \dagger}$ A. Sanchez-Hernandez, ${ }^{99, \dagger}$ S. Carrillo Moreno, ${ }^{100, \dagger}$ C. Oropeza Barrera,${ }^{100, \dagger}$ M. Ramirez-Garcia,${ }^{100, \dagger}$ F. Vazquez Valencia ${ }^{100, \dagger}$ J. Eysermans, ${ }_{101, \dagger}$ I. Pedraza,${ }^{101, \dagger}$ H. A. Salazar Ibarguen, ${ }^{101, \uparrow}$ C. Uribe Estrada, ${ }^{101, \dagger}$ A. Morelos Pineda, ${ }^{102, \dagger}$ J. Mijuskovic, ${ }^{103, e, \dagger}$ N. Raicevic,,${ }^{103, \uparrow}$ D. Krofcheck, ${ }^{104, \dagger}$ S. Bheesette, ${ }^{105, \dagger}$ P. H. Butler, ${ }^{105, \dagger}$ A. Ahmad, ${ }^{106, \dagger}$ M. I. Asghar, ${ }^{106, \dagger}$ M. I. M. Awan, ${ }_{106, \dagger}^{106 .}$ Q Hassan, ${ }^{106, \uparrow}$ H. R. Hoorani ${ }^{106, \dagger}$ W. A. Khan, ${ }^{106, \dagger}$ M. A. Shah, ${ }^{106, \dagger}$ M. Shoaib, ${ }_{106, \dagger}$ M. Waqas, ${ }_{106, \dagger}{ }^{\dagger}$ H. Bialkowska, ${ }^{108, \dagger}$ M. Bluj ${ }^{108, \dagger}$ B. Boimska, ${ }^{108, \dagger}$ T. Frueboes,${ }^{108, \dagger}$ M. Górski, ${ }^{108, \dagger}$ M. Kazana, ${ }^{108, \dagger}$ M. Szleper, ${ }^{108, \dagger}$ P. Traczyk, ${ }^{108, \uparrow}$ P. Zalewski, ${ }^{108, \uparrow}$ K. Bunkowski, ${ }^{109, \dagger}$ A. Byszuk, ${ }^{109, s s, \uparrow}$ K. Doroba,${ }^{109, \dagger}$ A. Kalinowski, ${ }^{109, \dagger}$ M. Konecki, ${ }^{109, \dagger}$ J. Krolikowski, ${ }^{109, \dagger}$ 
M. Olszewski, ${ }^{109, \dagger}$ M. Walczak, ${ }^{109, \dagger}$ M. Araujo, ${ }^{110, \dagger}$ P. Bargassa, ${ }^{110, \dagger}$ D. Bastos, ${ }^{110, \dagger}$ P. Faccioli, ${ }^{110, \dagger}$ M. Gallinaro, ${ }^{110, \dagger}$ J. Hollar, ${ }^{110, \dagger}$ N. Leonardo, ${ }^{110, \dagger}$ T. Niknejad, ${ }^{110, \dagger}$ J. Seixas, ${ }^{110, \dagger}$ K. Shchelina, ${ }^{110, \dagger}$ O. Toldaiev, ${ }^{110, \dagger}$ J. Varela, ${ }^{110, \uparrow}$ S. Afanasiev, ${ }^{111, \dagger}$ A. Baginyan, ${ }^{111, \dagger}$ P. Bunin, ${ }^{111, \dagger}$ A. Golunov, ${ }^{11, \dagger}$ I. Golutvin, ${ }^{111, \dagger}$ I. Gorbunov, ${ }^{11, \dagger}$ A. Kamenev, ${ }^{111, \dagger}$ V. Karjavine, ${ }^{111, \dagger}$ I. Kashunin, ${ }^{111, \dagger}$ V. Korenkov, ${ }^{111, \dagger}$ A. Lanev, ${ }^{111, \dagger}$ A. Malakhov, ${ }^{111, \dagger}$ V. Matveev, ${ }^{111, t t, u u, \dagger}$ P. Moisenz, ${ }^{111, \dagger}$ V. Palichik, ${ }^{111, \dagger}$ V. Perelygin, ${ }^{111, \dagger}$ M. Savina, ${ }^{111, \dagger}$ S. Shmatov, ${ }^{11, \dagger}$ S. Shulha, ${ }^{111, \dagger}$ V. Smirnov, ${ }^{111, \dagger}$ O. Teryaev, ${ }^{111, \dagger}$ N. Voytishin, ${ }^{111, \dagger}$ A. Zarubin, ${ }^{111, \uparrow}$ G. Gavrilov, ${ }^{112, \dagger}$ V. Golovtcov, ${ }^{112, \uparrow}$ Y. Ivanov, ${ }^{11, \dagger}$ V. Kim, ${ }^{112, v v, \uparrow}$ E. Kuznetsova, ${ }^{112, w w, \dagger}$ V. Murzin, ${ }^{112, \dagger}$ V. Oreshkin, ${ }^{112, \dagger}$ I. Smirnov, ${ }^{112, \dagger}$ D. Sosnov, ${ }^{112, \dagger}$ V. Sulimov, ${ }^{112, \dagger}$ L. Uvarov, ${ }^{112, \dagger}$ S. Volkov, ${ }^{112, \dagger}$ A. Vorobyev, ${ }^{112, \dagger}$ Yu. Andreev, ${ }^{113, \dagger}$ A. Dermenev, ${ }^{113, \dagger}$ S. Gninenko, ${ }^{113, \dagger}$ N. Golubev, ${ }^{113, \dagger}$ A. Karneyeu, ${ }^{113, \dagger}$ M. Kirsanov, ${ }^{113, \dagger}$ N. Krasnikov, ${ }^{11, \dagger}$ A. Pashenkov, ${ }^{113, \dagger}$ G. Pivovarov, ${ }^{113, \dagger}$ D. Tlisov, ${ }^{113, \dagger}$ A. Toropin, ${ }^{113, \dagger}$ V. Epshteyn, ${ }^{114, \dagger}$ V. Gavrilov, ${ }^{114, \dagger}$ N. Lychkovskaya, ${ }^{114, \dagger}$ A. Nikitenko, ${ }^{114, x x, \dagger}$ V. Popov, ${ }^{114, \dagger}$ I. Pozdnyakov, ${ }^{114, \dagger}$ G. Safronov, ${ }^{114, \dagger}$ A. Spiridonov, ${ }^{114, \dagger}$ A. Stepennov, ${ }^{114, \dagger}$ M. Toms, ${ }^{114, \dagger}$ E. Vlasov, ${ }^{114, \dagger}$ A. Zhokin, ${ }^{114, \dagger}$ T. Aushev, ${ }^{115, \dagger}$ O. Bychkova, ${ }^{116, \dagger}$ M. Chadeeva, ${ }^{116, y y, \dagger}$ D. Philippov, ${ }^{116, \dagger}$ E. Popova, ${ }^{116, \dagger}$ V. Rusinov, ${ }^{116, \dagger}$ V. Andreev, ${ }^{117, \dagger}$ M. Azarkin, ${ }^{117, \dagger}$ I. Dremin, ${ }^{117, \dagger}$ M. Kirakosyan, ${ }^{117, \dagger}$ A. Terkulov, ${ }^{117, \dagger}$ A. Belyaev, ${ }^{118, \dagger}$ E. Boos, ${ }^{118, \dagger}$ A. Ershov, ${ }^{118, \dagger}$ A. Gribushin, ${ }^{118, \dagger}$ L. Khein, ${ }^{118, \dagger}$ V. Klyukhin, ${ }^{118, \dagger}$ O. Kodolova, ${ }^{118, \dagger}$ I. Lokhtin, ${ }^{118, \dagger}$ O. Lukina, ${ }^{118, \dagger}$ S. Obraztsov, ${ }^{118, \dagger}$ S. Petrushanko, ${ }^{118, \dagger}$ V. Savrin, ${ }^{118, \dagger}$ A. Snigirev, ${ }^{118, \dagger}$ V. Blinov, ${ }^{119, z z, \dagger}$ T. Dimova, ${ }^{119, z z, \dagger}$ L. Kardapoltsev, ${ }^{119, z z, \dagger}$ I. Ovtin, ${ }^{119, z z, \dagger}$ Y. Skovpen, ${ }^{119, z z, \dagger}$ I. Azhgirey, ${ }^{120, \dagger}$ I. Bayshev, ${ }^{120, \dagger}$ V. Kachanov, ${ }^{120, \dagger}$ A. Kalinin, ${ }^{120, \dagger}$ D. Konstantinov, ${ }^{120, \dagger}$ V. Petrov, ${ }^{120, \dagger}$ R. Ryutin, ${ }^{120, \dagger}$ A. Sobol, ${ }^{120, \dagger}$ S. Troshin, ${ }^{120, \dagger}$ N. Tyurin, ${ }^{120, \dagger}$ A. Uzunian, ${ }^{120, \dagger}$ A. Volkov, ${ }^{120, \dagger}$ A. Babaev, ${ }^{121, \dagger}$ A. Iuzhakov, ${ }^{121, \dagger}$ V. Okhotnikov, ${ }^{121, \dagger}$ L. Sukhikh, ${ }^{121, \dagger}$ P. Adzic, ${ }^{123, a a a, \dagger}$ P. Cirkovic, ${ }^{123, \dagger}$ M. Dordevic, ${ }^{123, \dagger}$ P. Milenovic, ${ }^{123, \dagger}$ J. Milosevic, ${ }^{123, \dagger}$ M. Aguilar-Benitez, ${ }^{124, \dagger}$ J. Alcaraz Maestre, ${ }^{124, \dagger}$ A. Álvarez Fernández, ${ }^{124, \dagger}$ I. Bachiller, ${ }^{124, \dagger}$ M. Barrio Luna, ${ }^{124, \dagger}$ Cristina F. Bedoya, ${ }^{124, \dagger}$ J. A. Brochero Cifuentes, ${ }^{124, \dagger}$ C. A. Carrillo Montoya, ${ }^{124, \dagger}$ M. Cepeda, ${ }^{124, \dagger}$ M. Cerrada, ${ }^{124, \dagger}$ N. Colino, ${ }^{124, \dagger}$ B. De La Cruz, ${ }^{124, \dagger}$ A. Delgado Peris, ${ }^{124, \dagger}$ J. P. Fernández Ramos, ${ }^{124, \dagger}{ }^{\text {J. Flix }},{ }^{124, \dagger}$ M. C. Fouz, ${ }^{124, \dagger}$ A. García Alonso, ${ }^{124, \dagger}$ O. Gonzalez Lopez, ${ }^{124, \dagger}$ S. Goy Lopez, ${ }^{124, \dagger}$ J. M. Hernandez, ${ }^{124, \dagger}$ M. I. Josa, ${ }^{124, \dagger}$ D. Moran, ${ }^{124, \dagger}$ Á. Navarro Tobar, ${ }^{124, \dagger}$ A. Pérez-Calero Yzquierdo, ${ }^{124, \dagger}$ J. Puerta Pelayo, ${ }^{124, \dagger}$ I. Redondo, ${ }^{124, \dagger}$ L. Romero, ${ }^{124, \dagger}$ S. Sánchez Navas, ${ }^{124, \dagger}$ M. S. Soares, ${ }^{124, \dagger}$ A. Triossi, ${ }^{124, \dagger}$ C. Willmott, ${ }^{124, \dagger}$ C. Albajar, ${ }^{125, \dagger}$ J. F. de Trocóniz, ${ }^{125, \dagger}$ R. Reyes-Almanza, ${ }^{125, \dagger}$ B. Alvarez Gonzalez, ${ }^{126, \dagger}$ J. Cuevas, ${ }^{126, \dagger}$ C. Erice ${ }^{126, \dagger}$ J. Fernandez Menendez, ${ }^{126, \dagger}$ S. Folgueras, ${ }^{126, \dagger}$

I. Gonzalez Caballero, ${ }^{126, \dagger}$ E. Palencia Cortezon, ${ }^{126, \dagger}$ C. Ramón Álvarez, ${ }^{126, \dagger}$ V. Rodríguez Bouza, ${ }^{126, \dagger}$ S. Sanchez Cruz, ${ }^{126, \dagger}$ A. Trapote, ${ }^{126, \dagger}$ I. J. Cabrillo, ${ }^{127, \dagger}$ A. Calderon, ${ }^{127, \dagger}$ B. Chazin Quero, ${ }^{127, \dagger}$ J. Duarte Campderros, ${ }^{127, \dagger}$ M. Fernandez, ${ }^{127, \dagger}$ P. J. Fernández Manteca, ${ }^{127, \dagger}$ G. Gomez, ${ }^{127, \dagger}$ C. Martinez Rivero, ${ }^{127, \dagger}$ P. Martinez Ruiz del Arbol, ${ }^{127, \dagger}$ F. Matorras, ${ }^{127, \dagger}$ J. Piedra Gomez, ${ }^{127, \dagger}$ C. Prieels, ${ }^{127, \dagger}$ F. Ricci-Tam, ${ }^{127, \dagger}$ T. Rodrigo, ${ }^{127, \dagger}$ A. Ruiz-Jimeno, ${ }^{127, \dagger}$ L. Russo, ${ }^{127, b b b, \dagger}$ L. Scodellaro, ${ }^{127, \dagger}$ I. Vila, ${ }^{127, \dagger}$ J. M. Vizan Garcia, ${ }^{127, \dagger}$ MK Jayananda, ${ }^{128, \dagger}$ B. Kailasapathy, ${ }^{128, c c c, \dagger}$ D. U. J. Sonnadara, ${ }^{128, \dagger}$ DDC Wickramarathna, ${ }^{128, \dagger}$ W. G. D. Dharmaratna, ${ }^{129, \dagger}$ K. Liyanage, ${ }^{129, \dagger}$ N. Perera, ${ }^{129, \dagger}$ N. Wickramage, ${ }^{129, \dagger}$ T. K. Aarrestad, ${ }^{130, \dagger}$ D. Abbaneo, ${ }^{130, \dagger}$ B. Akgun, ${ }^{130, \dagger}$ E. Auffray, ${ }^{130, \dagger}$ G. Auzinger, ${ }^{130, \dagger}$ P. Baillon, ${ }^{130, \dagger}$ A. H. Ball, ${ }^{130, \dagger}$

D. Barney, ${ }^{130, \dagger}$ J. Bendavid, ${ }^{130, \dagger}$ N. Beni, ${ }^{130, \dagger}$ M. Bianco, ${ }^{130, \dagger}$ A. Bocci, ${ }^{130, \dagger}$ P. Bortignon, ${ }^{130, \dagger}$ E. Brondolin, ${ }^{130, \dagger}$ T. Camporesi, ${ }^{130, \dagger}$ G. Cerminara, ${ }^{130, \dagger}$ L. Cristella, ${ }^{130, \dagger}$ D. d'Enterria, ${ }^{130, \dagger}$ A. Dabrowski, ${ }^{130, \dagger}$ N. Daci, ${ }^{130, \dagger}$ V. Daponte, ${ }^{130, \dagger}$ A. David, ${ }^{130, \dagger}$ A. De Roeck, ${ }^{130, \dagger}$ R. Di Maria, ${ }^{130, \dagger}$ M. Dobson, ${ }^{130, \dagger}$ M. Dünser, ${ }^{130, \dagger}$ N. Dupont, ${ }^{130, \dagger}$ A. Elliott-Peisert, ${ }^{130, \dagger}$ N. Emriskova, ${ }^{130, \dagger}$ F. Fallavollita, ${ }^{130, d d d, \dagger}$ D. Fasanella, ${ }^{130, \dagger}$ S. Fiorendi, ${ }^{130, \dagger}$ G. Franzoni, ${ }^{130, \dagger}$ J. Fulcher, ${ }^{130, \dagger}$ W. Funk, ${ }^{130, \dagger}$ D. Gigi, ${ }^{130, \dagger}$ K. Gill, ${ }^{130, \dagger}$ F. Glege,${ }^{130, \dagger}$ L. Gouskos, ${ }^{130,{ }^{\dagger}}$ M. Guilbaud, ${ }^{130, \dagger}$ D. Gulhan, ${ }^{130, \dagger}$ M. Haranko, ${ }^{130, \dagger}$ J. Hegeman, ${ }^{130, \dagger}$ Y. Iiyama, ${ }^{130, \dagger}$ V. Innocente,${ }^{130, \dagger}$ T. James,${ }^{130, \dagger}$ P. Janot,${ }^{130, \dagger}$ J. Kieseler, ${ }^{130, \dagger}$ M. Komm, ${ }^{130, \dagger}$ N. Kratochwil, ${ }^{130, \dagger}$ C. Lange, ${ }^{130, \dagger}$

P. Lecoq, ${ }^{130, \dagger}$ K. Long, ${ }^{130, \dagger}$ C. Lourenço, ${ }^{130, \dagger}$ L. Malgeri, ${ }^{130, \dagger}$ M. Mannelli, ${ }^{130, \dagger}$ A. Massironi, ${ }^{130, \dagger}$ F. Meijers, ${ }^{130, \dagger}$ S. Mersi, ${ }^{130, \dagger}$ E. Meschi, ${ }^{130, \dagger}$ F. Moortgat, ${ }^{130, \dagger}$ M. Mulders, ${ }^{130, \dagger}$ J. Ngadiuba, ${ }^{130, \dagger}$ J. Niedziela, ${ }^{130, \dagger}$ S. Orfanelli, ${ }^{130, \dagger}$ L. Orsini, ${ }^{130, \dagger}$ F. Pantaleo, ${ }^{130, \mathrm{u}, \dagger}$ L. Pape,${ }^{130, \dagger}$ E. Perez, ${ }^{130, \dagger}$ M. Peruzzi, ${ }^{130, \dagger}$ A. Petrilli, ${ }^{130, \dagger}$ G. Petrucciani, ${ }^{130, \dagger}$ A. Pfeiffer, ${ }^{130, \dagger}$ M. Pierini, ${ }^{130, \dagger}$ D. Rabady, ${ }^{130, \dagger}$ A. Racz, ${ }^{130, \dagger}$ M. Rieger, ${ }^{130, \dagger}$ M. Rovere, ${ }^{130, \dagger}$ H. Sakulin, ${ }^{130, \dagger}$ J. Salfeld-Nebgen, ${ }^{130, \dagger}$ S. Scarfi, ${ }^{130, \dagger}$ C. Schäfer, ${ }^{130, \dagger}$ C. Schwick, ${ }^{130, \dagger}$ M. Selvaggi, ${ }^{130, \dagger}$ A. Sharma, ${ }^{130, \dagger}$ P. Silva, ${ }^{130, \dagger}$ P. Sphicas, ${ }^{130, e e e, \dagger}$ J. Steggemann, ${ }^{130, \dagger}$ S. Summers, ${ }^{130, \dagger}$ V. R. Tavolaro, ${ }^{130, \dagger}$ D. Treille, ${ }^{130, \dagger}$ A. Tsirou, ${ }^{130, \dagger}$ G. P. Van Onsem, ${ }^{130, \dagger}$ A. Vartak, ${ }^{130, \dagger}$ M. Verzetti, ${ }^{130, \dagger}$ K. A. Wozniak, ${ }^{130, \dagger}$ W. D. Zeuner, ${ }^{130, \dagger}$ L. Caminada, ${ }^{131, f f f, \dagger}$ W. Erdmann, ${ }^{131, \dagger}$ R. Horisberger, ${ }^{131, \dagger}$ Q. Ingram, ${ }^{131, \dagger}$ H. C. Kaestli, ${ }^{131, \dagger}$ D. Kotlinski, ${ }^{131, \dagger}$ U. Langenegger, ${ }^{131, \dagger}$ T. Rohe, ${ }^{131, \dagger}$ M. Backhaus, ${ }^{132, \dagger}$ P. Berger, ${ }^{132, \dagger}$ A. Calandri, ${ }^{132, \dagger}$ N. Chernyavskaya, ${ }^{132, \dagger}$ G. Dissertori, ${ }^{132, \dagger}$ M. Dittmar, ${ }^{132, \dagger}$ M. Donegà, ${ }^{132, \dagger}$ C. Dorfer, ${ }^{132, \dagger}$ T. Gadek, ${ }^{132, \dagger}$ T. A. Gómez Espinosa, ${ }^{132, \dagger}$ C. Grab, ${ }^{132, \dagger}$ D. Hits, ${ }^{132, \dagger}$ W. Lustermann, ${ }^{132, \dagger}$ A.-M. Lyon, ${ }^{132, \dagger}$ R. A. Manzoni, ${ }^{132, \dagger}$ M. T. Meinhard, ${ }^{132, \dagger}$ F. Micheli, ${ }^{132, \dagger}$ F. Nessi-Tedaldi, ${ }^{132, \dagger}$ F. Pauss, ${ }^{132, \dagger}$ V. Perovic, ${ }^{132, \dagger}$ G. Perrin, ${ }^{132, \dagger}$ L. Perrozzi, ${ }^{132, \dagger}$ 
S. Pigazzini ${ }^{132, \dagger}$ M. G. Ratti, ${ }^{132, \dagger}$ M. Reichmann, ${ }^{132, \dagger}$ C. Reissel,${ }^{132, \dagger}$ T. Reitenspiess, ${ }^{132, \dagger}$ B. Ristic, ${ }^{132, \dagger}$ D. Ruini, ${ }^{132, \uparrow}$ D. A. Sanz Becerra, ${ }^{132, \dagger}$ M. Schönenberger, ${ }^{132, \dagger}$ L. Shchutska, ${ }^{132, \dagger}$ V. Stampf,${ }^{132, \dagger}$ M. L. Vesterbacka Olsson, ${ }^{132, \dagger}$ R. Wallny, ${ }^{132, \uparrow}$ D. H. Zhu, ${ }^{132, \uparrow}$ C. Amsler, ${ }^{133, g g g, \dagger}$ C. Botta,${ }^{133, \uparrow}$ D. Brzhechko, ${ }^{133, \dagger}$ M. F. Canelli, ${ }^{133, \dagger}$ A. De Cosa, ${ }^{133, \uparrow}$ R. Del Burgo, ${ }^{133, \dagger}$ J. K. Heikkilä, ${ }^{133, \dagger}$ M. Huwiler, ${ }^{133, \dagger}$ A. Jofrehei, ${ }^{133, \dagger}$ B. Kilminster, ${ }^{133, \dagger}$ S. Leontsinis, ${ }^{133, \dagger}$ A. Macchiolo, ${ }^{133, \dagger}$ P. Meiring, ${ }^{133, \dagger}$ V. M. Mikuni, ${ }^{133, \dagger}$ U. Molinatti, ${ }^{133, \dagger}$ I. Neutelings, ${ }^{133, \dagger}$ G. Rauco, ${ }^{133, \dagger}$ A. Reimers,${ }^{133, \uparrow}$ P. Robmann, ${ }^{133, \dagger}$ K. Schweiger, ${ }^{133, \dagger}$ Y. Takahashi, ${ }^{133, \dagger}$ S. Wertz ${ }^{133, \dagger}$ C. Adloff, ${ }^{134, \text { hhh, } \dagger}$ C. M. Kuo, ${ }^{134, \dagger}$ W. Lin, ${ }^{134, \dagger}$ A. Roy, ${ }^{134, \dagger}$ T. Sarkar, ${ }^{134, j \mathrm{j}, \dagger}$ S. S. Yu ${ }^{134, \dagger}$ L. Ceard,${ }^{135, \dagger}$ P. Chang, ${ }^{135, \dagger}$ Y. Chao, ${ }^{135, \dagger}$ K. F. Chen, ${ }^{135, \dagger}$ P. H. Chen, ${ }^{135, \dagger}$ W.-S. Hou, ${ }^{135, \dagger}$ Y. y. Li,${ }^{135, \dagger}$ R.-S. Lu, ${ }^{135, \dagger}$ E. Paganis,${ }^{135, \dagger}$ A. Psallidas, ${ }^{135, \uparrow}$ A. Steen,${ }^{135, \dagger}$ E. Yazgan, ${ }^{135, \dagger}$ B. Asavapibhop,${ }^{136, \uparrow}$ C. Asawatangtrakuldee, ${ }^{136, \dagger}$ N. Srimanobhas, ${ }^{136, \dagger}$ F. Boran, ${ }^{137, \dagger}$ S. Damarseckin,${ }^{137, \mathrm{iii}, \dagger}$ Z. S. Demiroglu, ${ }^{137, \dagger}$ F. Dolek, ${ }^{137, \uparrow}$

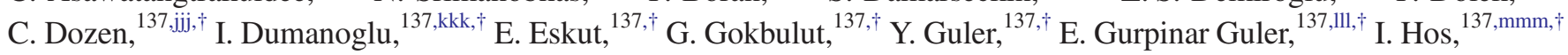
C. Isik, ${ }^{137, \dagger}$ E. E. Kangal, ${ }^{137, \text { nnn, } \uparrow}$ O. Kara, ${ }^{137, \uparrow}$ A. Kayis Topaksu, ${ }^{137, \uparrow}$ U. Kiminsu, ${ }^{137, \uparrow}$ G. Onengut, ${ }^{137, \dagger}$ K. Ozdemir, ${ }^{137,000, \uparrow}$ A. Polatoz, ${ }^{137, \dagger}$ A. E. Simsek, ${ }^{137, \dagger}$ B. Tali, ${ }^{137, p p p, \dagger}$ U. G. Tok, ${ }^{137, \dagger}$ S. Turkcapar, ${ }^{137, \dagger}$ I. S. Zorbakir, ${ }^{137, \dagger}$ C. Zorbilmez, ${ }^{137, \dagger}$

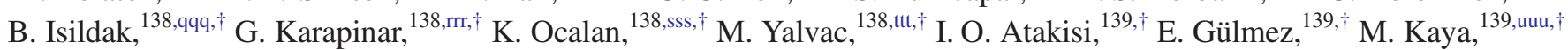
O. Kaya ${ }^{139, v v v, \uparrow}$ Ö. Özçelik, ${ }^{139, \dagger}$ S. Tekten, ${ }^{139, w w w, \dagger}$ E. A. Yetkin, ${ }^{139, x x x, \dagger}$ A. Cakir, ${ }^{140,{ }^{\dagger}}$ K. Cankocak, ${ }^{140, k k k, \uparrow}$ Y. Komurcu, ${ }^{140,{ }^{\dagger}}$ S. Sen, ${ }^{140, y y y, \dagger}$ F. Aydogmus Sen ${ }^{141, \dagger}$ S. Cerci,${ }^{141, p p p, \dagger}$ S. Ozkorucuklu, ${ }^{141, \dagger}$ D. Sunar Cerci, ${ }^{141, p p p, \dagger}$ B. Grynyov, ${ }^{142, \uparrow}$ L. Levchuk ${ }^{143, \dagger}$ E. Bhal ${ }^{144, \uparrow}$ S. Bologna, ${ }^{144, \dagger}$ J. J. Brooke, ${ }^{144, \uparrow}$ E. Clement,${ }^{144, \dagger}$ D. Cussans, ${ }^{144, \dagger}$ H. Flacher, ${ }^{144, \uparrow}$ J. Goldstein, ${ }^{144, \dagger}$ G. P. Heath, ${ }^{144, \dagger}$ H. F. Heath, ${ }^{144, \dagger}$ L. Kreczko, ${ }^{144, \dagger}$ B. Krikler, ${ }^{144, \dagger}$ S. Paramesvaran, ${ }^{144, \dagger}$ T. Sakuma, ${ }^{144, \uparrow}$ S. Seif El Nasr-Storey, ${ }^{144, \dagger}$ V. J. Smith, ${ }^{144, \dagger}$ J. Taylor, ${ }^{144, \dagger}$ A. Titterton, ${ }^{144, \uparrow}$ K. W. Bell, ${ }^{145, \dagger}$ A. Belyaev, ${ }^{145, z z z, \dagger}$ C. Brew, ${ }^{145, \uparrow}$ R. M. Brown, ${ }^{145, \dagger}$ D. J. A. Cockerill, ${ }^{145, \dagger}$ K. V. Ellis, ${ }^{145, \dagger}$ K. Harder, ${ }^{145, \dagger}$ S. Harper, ${ }^{145, \dagger}$ J. Linacre,,${ }^{145, \dagger}$ K. Manolopoulos,${ }^{145, \uparrow}$ D. M. Newbold ${ }^{145, \dagger}$ E. Olaiya, ${ }^{145, \dagger}$ D. Petyt,${ }^{145, \dagger}$ T. Reis, ${ }^{145, \dagger}$ T. Schuh,,${ }^{145, \dagger}$ C. H. Shepherd-Themistocleous, ${ }^{145, \dagger}$

A. Thea, ${ }^{145, \dagger}$ I. R. Tomalin, ${ }^{145, \uparrow}$ T. Williams,${ }^{145, \uparrow}$ R. Bainbridge, ${ }^{146, \dagger}$ P. Bloch,${ }^{146, \uparrow}$ S. Bonomally, ${ }^{146, \uparrow}$ J. Borg, ${ }^{146, \uparrow}$ S. Breeze, ${ }^{146, \uparrow}$ O. Buchmuller, ${ }^{146, \uparrow}$ A. Bundock, ${ }^{146, \dagger}$ V. Cepaitis, ${ }^{146, \uparrow}$ G. S. Chahal, ${ }^{146, a a a, \uparrow}$ D. Colling, ${ }^{146, \dagger}$ P. Dauncey, ${ }^{146, \uparrow}$ G. Davies, ${ }^{146, \uparrow}$ M. Della Negra, ${ }^{146, \uparrow}$ P. Everaerts, ${ }^{146, \uparrow}$ G. Fedi,${ }^{146, \uparrow}$ G. Hall ${ }^{146, \uparrow}$ G. Iles, ${ }^{146, \uparrow}$ J. Langford ${ }^{146, \dagger}$ L. Lyons,${ }^{146, \uparrow}$ A.-M. Magnan, ${ }^{146, \uparrow}$ S. Malik, ${ }^{146, \uparrow}$ A. Martelli, ${ }^{146, \dagger}$ V. Milosevic, ${ }^{146, \dagger}$ J. Nash, ${ }^{146, b b b b, \dagger}$ V. Palladino,${ }^{146, \dagger}$ M. Pesaresi, ${ }^{146, \dagger}$ D. M. Raymond ${ }^{146, \dagger}$ A. Richards, ${ }^{146, \dagger}$ A. Rose,${ }^{146, \dagger}$ E. Scott,${ }^{146, \dagger}$ C. Seez, ${ }^{146, \dagger}$ A. Shtipliyski, ${ }^{146, \dagger}$ M. Stoye,${ }^{146, \uparrow}$

A. Tapper, ${ }^{146, \dagger}$ K. Uchida, ${ }^{146, \dagger}$ T. Virdee,${ }^{146, \mathrm{u}, \dagger}$ N. Wardle, ${ }^{146, \dagger}$ S. N. Webb ${ }^{146, \dagger}$ D. Winterbottom, ${ }^{146, \dagger}$ A. G. Zecchinelli, ${ }^{146, \uparrow}$ S. C. Zenz, ${ }^{146, \dagger}$ J. E. Cole,${ }^{147, \dagger}$ P. R. Hobson,${ }^{147, \dagger}$ A. Khan,${ }^{147, \dagger}$ P. Kyberd, ${ }^{147, \dagger}$ C. K. Mackay, ${ }^{147, \dagger}$ I. D. Reid, ${ }^{147, \dagger}$

L. Teodorescu, ${ }^{147, \uparrow}$ S. Zahid, ${ }^{147, \uparrow}$ A. Brinkerhoff, ${ }^{148, \uparrow}$ K. Call, ${ }^{148, \uparrow}$ B. Caraway, ${ }^{148, \uparrow}$ J. Dittmann, ${ }^{148, \dagger}$ K. Hatakeyama, ${ }^{148, \uparrow}$ A. R. Kanuganti, ${ }^{148, \dagger}$ C. Madrid, ${ }^{148, \dagger}$ B. McMaster,${ }^{148, \dagger}$ N. Pastika, ${ }^{148, \dagger}$ S. Sawant, ${ }^{148, \dagger}$ C. Smith, ${ }^{148, \dagger}$ R. Bartek, ${ }^{149, \dagger}$ A. Dominguez, ${ }^{149, \dagger}$ R. Uniyal, ${ }^{149, \dagger}$ A. M. Vargas Hernandez, ${ }^{149, \dagger}$ A. Buccilli, ${ }^{150, \dagger}$ O. Charaf, ${ }^{150, \dagger}$ S. I. Cooper, ${ }^{150, \dagger}$ S. V. Gleyzer ${ }^{150, \dagger}$ C. Henderson, ${ }^{150, \dagger}$ P. Rumerio, ${ }^{150, \dagger}$ C. West,${ }^{150, \dagger}$ A. Akpinar, ${ }^{151, \dagger}$ A. Albert, ${ }^{151, \dagger}$ D. Arcaro, ${ }^{151, \dagger}$ C. Cosby ${ }_{1}^{151, \dagger}$ Z. Demiragli, ${ }^{151, \dagger}$ D. Gastler ${ }^{151, \dagger}$ C. Richardson, ${ }^{151, \dagger}$ J. Rohlf, ${ }^{151, \dagger}$ K. Salyer, ${ }^{151, \dagger}$ D. Sperka, ${ }^{151, \dagger}$ D. Spitzbart, ${ }^{151, \dagger}$ I. Suarez,${ }^{151, \dagger}$ S. Yuan, ${ }^{151, \dagger}$ D. Zou, ${ }^{151, \dagger}$ G. Benelli, ${ }^{152, \dagger}$ B. Burkle, ${ }^{152, \dagger}$ X. Coubez, ${ }^{152, v, \dagger}$ D. Cutts, ${ }^{152, \uparrow}$ Y. t. Duh, ${ }^{152, \uparrow}$ M. Hadley, ${ }^{152, \dagger}$ U. Heintz, ${ }^{152, \dagger}$ J. M. Hogan, ${ }^{152, \mathrm{cccc}, \dagger}$ K. H. M. Kwok, ${ }^{152, \dagger}$ E. Laird,${ }^{152, \dagger}$ G. Landsberg, ${ }^{152, \dagger}$ K. T. Lau,${ }^{152, \dagger}$ J. Lee,${ }^{152, \dagger}$ M. Narain, ${ }^{152, \dagger}$ S. Sagir, ${ }^{152, \text { dddd, },}$ R. Syarif, ${ }^{152, \dagger}$ E. Usai, ${ }^{152, \dagger}$ W. Y. Wong, ${ }^{152, \dagger}$ D. Yu, ${ }^{152, \dagger}$ W. Zhang, ${ }^{152, \dagger}$ R. Band,${ }^{153, \dagger}$ C. Brainerd, ${ }^{153, \dagger}$ R. Breedon, ${ }^{153, \dagger}$ M. Calderon De La Barca Sanchez, ${ }^{153, \dagger}$ M. Chertok, ${ }^{153, \dagger}$ J. Conway, ${ }^{153, \dagger}$ R. Conway, ${ }^{153, \dagger}$ P. T. Cox,${ }^{153, \dagger}$ R. Erbacher, ${ }^{153, \dagger}$ C. Flores,${ }^{153, \dagger}$ G. Funk,${ }^{153, \dagger}$ F. Jensen, ${ }^{153, \dagger}$ W. Ko, ${ }^{153, a, \dagger}$ O. Kukral, ${ }^{153, \dagger}$ R. Lander, ${ }^{153, \dagger}$ M. Mulhearn, ${ }^{153, \dagger}$ D. Pellett, ${ }^{153, \dagger}$ J. Pilot, ${ }^{153, \dagger}$ M. Shi, ${ }^{153, \dagger}$ D. Taylor, ${ }^{153, \dagger}$ K. Tos, ${ }^{153, \dagger}$ M. Tripathi, ${ }^{153, \dagger}$ Y. Yao, ${ }^{153, \dagger}$ F. Zhang, ${ }^{153, \dagger}$ M. Bachtis,${ }^{154, \dagger}$ R. Cousins,${ }^{154, \dagger}$ A. Dasgupta, ${ }^{154, \dagger}$ A. Florent,${ }^{154, \uparrow}$ D. Hamilton, ${ }^{154, \dagger}$ J. Hauser ${ }^{154, \dagger}$ M. Ignatenko, ${ }^{154, \dagger}$ T. Lam, ${ }^{154, \dagger}$ N. Mccoll, ${ }^{154, \dagger}$ W. A. Nash, ${ }^{154, \dagger}$ S. Regnard, ${ }^{154, \dagger}$ D. Saltzberg, ${ }^{154, \dagger}$ C. Schnaible, ${ }^{154, \dagger}$ B. Stone ${ }^{154, \uparrow}$ V. Valuev, ${ }^{154, \dagger}$ K. Burt, ${ }^{155, \dagger}$ Y. Chen, ${ }^{155, \uparrow}$ R. Clare, ${ }^{15, \dagger}$ J. W. Gary, ${ }^{155, \uparrow}$ S. M. A. Ghiasi Shirazi, ${ }^{155, \dagger}$ G. Hanson, ${ }^{155, \dagger}$ G. Karapostoli, ${ }^{155, \dagger}$ O. R. Long, ${ }^{155, \uparrow}$ N. Manganelli, ${ }^{155, \uparrow}$

M. Olmedo Negrete, ${ }^{155, \dagger}$ M. I. Paneva, ${ }^{155, \dagger}$ W. Si ${ }^{155, \dagger}$ S. Wimpenny, ${ }^{15, \dagger}$ Y. Zhang, ${ }_{15, \uparrow}^{156}$ J. G. Branson, ${ }^{156, \dagger}$ P. Chang ${ }^{156, \uparrow}$ S. Cittolin, ${ }^{156, \dagger}$ S. Cooperstein, ${ }^{156, \uparrow}$ N. Deelen, ${ }^{156, \dagger}$ M. Derdzinski, ${ }^{156, \dagger}$ J. Duarte, ${ }^{156, \dagger}$ R. Gerosa, ${ }^{156, \uparrow}$ D. Gilbert, ${ }^{15, \dagger}$ B. Hashemi ${ }^{156, \dagger}$ D. Klein,${ }^{156, \dagger}$ V. Krutelyov ${ }^{156, \uparrow}$ J. Letts, ${ }^{156, \dagger}$ M. Masciovecchio ${ }^{156, \uparrow}$ S. May, ${ }^{156, \uparrow}$ S. Padhi, ${ }^{156, \dagger}$ M. Pieri, ${ }^{156, \uparrow}$ V. Sharma ${ }^{156, \dagger}$ M. Tadel ${ }^{156, \dagger}$ F. Würthwein,${ }^{156, \dagger}$ A. Yagil,${ }^{156, \dagger}$ N. Amin, ${ }^{157, \dagger}$ C. Campagnari, ${ }^{157, \dagger}$ M. Citron, ${ }^{157, \dagger}$

A. Dorsett, ${ }^{157, \dagger}$ V. Dutta, ${ }^{157, \dagger}$ J. Incandela, ${ }^{157, \dagger}$ B. Marsh ${ }^{157, \dagger}$ H. Mei, ${ }^{157, \dagger}$ A. Ovcharova, ${ }^{157, \dagger}$ H. Qu, ${ }^{157, \dagger}$ M. Quinnan, ${ }^{157, \uparrow}$ J. Richman, ${ }^{157, \dagger}$ U. Sarica, ${ }^{157, \dagger}$ D. Stuart, ${ }^{157, \dagger}$ S. Wang, ${ }^{157, \dagger}$ D. Anderson, ${ }^{158, \dagger}$ A. Bornheim, ${ }^{158, \dagger}$ O. Cerri, ${ }^{158, \dagger}$ I. Dutta, ${ }^{158, \uparrow}$ J. M. Lawhorn, ${ }^{158, \dagger}$ N. Lu, ${ }^{158, \dagger}$ J. Mao, ${ }^{158, \uparrow}$ H. B. Newman, ${ }^{158, \dagger}$ T. Q. Nguyen, ${ }^{158, \uparrow}$ J. Pata, ${ }^{158, \dagger}$ M. Spiropulu, ${ }^{158, \uparrow}$ 
J. R. Vlimant ${ }^{158, \uparrow}$ S. Xie ${ }^{158, \uparrow}$ Z. Zhang, ${ }^{158, \uparrow}$ R. Y. Zhu, ${ }^{158, \uparrow}$ J. Alison,${ }^{159, \dagger}$ M. B. Andrews, ${ }^{159, \dagger}$ T. Ferguson, ${ }^{159, \dagger}$ T. Mudholkar, ${ }^{159, \dagger}$ M. Paulini, ${ }^{159, \dagger}$ M. Sun, ${ }^{159, \dagger}$ I. Vorobiev, ${ }^{159, \dagger}$ J. P. Cumalat, ${ }^{160, \uparrow}$ W. T. Ford, ${ }^{160, \dagger}$ E. MacDonald, ${ }^{160, \uparrow}$ T. Mulholland, ${ }^{160, \dagger}$ R. Patel, ${ }^{160, \dagger}$ A. Perloff, ${ }^{160, \dagger}$ K. Stenson, ${ }^{160, \dagger}$ K. A. Ulmer, ${ }^{160, \dagger}$ S. R. Wagner, ${ }^{160, \dagger}$ J. Alexander, ${ }^{161, \dagger}$ Y. Cheng, ${ }^{161, \dagger}$ J. Chu, ${ }^{161, \dagger}$ D. J. Cranshaw, ${ }^{161, \dagger}$ A. Datta, ${ }^{161, \dagger}$ A. Frankenthal, ${ }^{161, \dagger}$ K. Mcdermott, ${ }^{161, \dagger}$ J. Monroy, ${ }^{161, \dagger}$ J. R. Patterson, ${ }^{161, \dagger}$ D. Quach,${ }^{161, \dagger}$ A. Ryd ${ }^{161, \dagger}$ W. Sun ${ }^{161, \uparrow}$ S. M. Tan, ${ }^{161, \dagger}$ Z. Tao, ${ }^{161, \dagger}$ J. Thom, ${ }^{161, \dagger}$ P. Wittich, ${ }^{161, \dagger}$ M. Zientek, ${ }^{161, \dagger}$ S. Abdullin, ${ }^{162, \dagger}$ M. Albrow, ${ }^{162, \dagger}$ M. Alyari, ${ }^{162, \dagger}$ G. Apollinari, ${ }^{162, \dagger}$ A. Apresyan, ${ }^{162, \dagger}$ A. Apyan, ${ }^{162, \dagger}$ S. Banerjee, ${ }^{162, \dagger}$ L. A. T. Bauerdick, ${ }^{162, \dagger}$ A. Beretvas, ${ }^{162, \uparrow}$ D. Berry, ${ }^{162, \dagger}$ J. Berryhill, ${ }^{162, \dagger}$ P. C. Bhat, ${ }^{162, \dagger}$ K. Burkett, ${ }^{162, \dagger}$ J. N. Butler, ${ }^{162, \dagger}$ A. Canepa, ${ }^{162, \dagger}$ G. B. Cerati, ${ }^{162, \dagger}$ H. W. K. Cheung, ${ }^{162, \dagger}$ F. Chlebana, ${ }^{162, \dagger}$ M. Cremonesi, ${ }^{162, \dagger}$ V. D. Elvira, ${ }^{162, \dagger}$ J. Freeman,${ }^{162, \dagger}$ Z. Gecse,${ }^{162, \dagger}$ E. Gottschalk, ${ }^{162, \dagger}$ L. Gray,${ }^{162, \dagger}$ D. Green,${ }^{162, \dagger}$ S. Grünendahl, ${ }^{162, \dagger}$ O. Gutsche, ${ }^{162, \dagger}$ R. M. Harris, ${ }^{162, \dagger}$ S. Hasegawa, ${ }^{162, \dagger}$ R. Heller ${ }^{162, \dagger}$ T. C. Herwig, ${ }^{162, \dagger}$ J. Hirschauer, ${ }^{162, \dagger}$ B. Jayatilaka, ${ }^{162, \dagger}$ S. Jindariani, ${ }^{162, \dagger}$ M. Johnson, ${ }^{162, \dagger}$ U. Joshi, ${ }^{162, \dagger}$ T. Klijnsma, ${ }^{162, \dagger}$ B. Klima, ${ }^{162, \dagger}$ M. J. Kortelainen, ${ }^{162, \dagger}$ S. Lammel, ${ }^{162, \dagger}$ D. Lincoln, ${ }^{162, \dagger}$ R. Lipton, ${ }^{162, \dagger}$ M. Liu, ${ }^{162, \dagger}$ T. Liu, ${ }^{162, \dagger}$ J. Lykken, ${ }^{162, \dagger}$ K. Maeshima, ${ }^{162, \dagger}$ D. Mason, ${ }^{162, \dagger}$ P. McBride, ${ }^{162, \dagger}$ P. Merkel ${ }^{162, \dagger}$ S. Mrenna, ${ }^{162, \dagger}$ S. Nahn, ${ }^{162, \dagger}$ V. O’Dell, ${ }^{162, \dagger}$ V. Papadimitriou, ${ }^{162, \dagger}$ K. Pedro, ${ }^{162, \dagger}$ C. Pena, ${ }^{162, e e e, ~}{ }^{16}$ O. Prokofyev, ${ }^{162, \dagger}$ F. Ravera, ${ }^{162, \uparrow}$ A. Reinsvold Hall, ${ }^{162, \dagger}$ L. Ristori, ${ }^{162, \uparrow}$ B. Schneider, ${ }^{162, \uparrow}$ E. Sexton-Kennedy, ${ }^{162, \uparrow}$ N. Smith, ${ }^{162, \dagger}$ A. Soha, ${ }^{162, \dagger}$ W. J. Spalding, ${ }^{162, \dagger}$ L. Spiegel, ${ }^{162, \dagger}$ S. Stoynev, ${ }^{162, \dagger}$ J. Strait, ${ }^{162, \dagger}$ L. Taylor, ${ }^{162, \dagger}$ S. Tkaczyk, ${ }^{162, \uparrow}$ N. V. Tran, ${ }^{162, \dagger}$ L. Uplegger, ${ }^{162, \dagger}$ E. W. Vaandering, ${ }^{162, \uparrow}$ M. Wang, ${ }^{162, \dagger}$ H. A. Weber, ${ }^{162, \dagger}$ A. Woodard, ${ }^{162, \dagger}$ D. Acosta, ${ }^{163, \dagger}$ P. Avery, ${ }^{163, \dagger}$ D. Bourilkov, ${ }^{163, \dagger}$ L. Cadamuro, ${ }^{163, \dagger}$ V. Cherepanov, ${ }^{163, \dagger}$ F. Errico, ${ }^{163, \dagger}$ R. D. Field,${ }^{163, \uparrow}$ D. Guerrero, ${ }^{163, \uparrow}$ B. M. Joshi, ${ }^{163, \dagger}$ M. Kim, ${ }^{163, \uparrow}$ J. Konigsberg, ${ }^{163, \uparrow}$ A. Korytov, ${ }^{163, \dagger}$ K. H. Lo, ${ }^{163, \dagger}$ K. Matchev, ${ }^{163, \dagger}$ N. Menendez, ${ }^{163, \uparrow}$ G. Mitselmakher, ${ }^{163, \dagger}$ D. Rosenzweig, ${ }^{163, \dagger}$ K. Shi, ${ }^{163, \dagger}$ J. Wang, ${ }^{163, \dagger}$ S. Wang, ${ }^{163, \dagger}$ X. Zuo, ${ }^{163, \dagger}$ Y. R. Joshi, ${ }^{164, \dagger}$ T. Adams, ${ }^{165, \uparrow}$ A. Askew, ${ }^{165, \dagger}$ D. Diaz, ${ }^{165, \dagger}$ R. Habibullah, ${ }^{165, \dagger}$ S. Hagopian, ${ }^{165, \dagger}$ V. Hagopian, ${ }^{165, \dagger}$ K. F. Johnson, ${ }^{165, \uparrow}$ R. Khurana, ${ }^{165, \dagger}$

T. Kolberg, ${ }^{165, \uparrow}$ G. Martinez, ${ }^{165, \dagger}$ H. Prosper, ${ }^{165, \dagger}$ C. Schiber, ${ }^{165, \dagger}$ R. Yohay, ${ }^{165, \dagger}$ J. Zhang, ${ }^{165, \dagger}$ M. M. Baarmand, ${ }^{166, \dagger}$ S. Butalla, ${ }^{166, \dagger}$ T. Elkafrawy, ${ }^{166,0, \dagger}$ M. Hohlmann, ${ }^{166,{ }^{\dagger}}$ D. Noonan, ${ }^{166, \dagger}$ M. Rahmani, ${ }^{166, \dagger}$ M. Saunders, ${ }^{166, \dagger}$ F. Yumiceva, ${ }^{166, \uparrow}$ M. R. Adams, ${ }^{167, \dagger}$ L. Apanasevich, ${ }^{167, \dagger}$ H. Becerril Gonzalez, ${ }^{167, \dagger}$ R. Cavanaugh, ${ }^{167, \dagger}$ X. Chen, ${ }^{167, \dagger}$ S. Dittmer, ${ }^{167, \dagger}$ O. Evdokimov, ${ }^{167, \dagger}$ C. E. Gerber, ${ }^{167, \dagger}$ D. A. Hangal,${ }^{167, \dagger}$ D. J. Hofman, ${ }^{167, \dagger}$ C. Mills, ${ }^{167, \dagger}$ G. Oh, ${ }^{167, \dagger}$ T. Roy, ${ }^{167, \dagger}$ M. B. Tonjes ${ }^{167, \dagger}$ N. Varelas,${ }^{167, \dagger}$ J. Viinikainen, ${ }^{167, \dagger}$ H. Wang,${ }^{167, \dagger}$ X. Wang,${ }^{167, \dagger}$ Z. Wu,${ }^{167, \dagger}$ M. Alhusseini, ${ }^{168, \dagger}$ B. Bilki, ${ }^{168,111, \dagger}$ K. Dilsiz, ${ }^{168, f f f f, \dagger}$ S. Durgut,${ }^{168, \dagger}$ R. P. Gandrajula, ${ }^{168, \dagger}$ M. Haytmyradov, ${ }^{168, \dagger}$ V. Khristenko, ${ }^{168, \dagger}$ O. K. Köseyan, ${ }^{168, \dagger}$ J.-P. Merlo, ${ }^{168, \dagger}$ A. Mestvirishvili, ${ }^{168, g g g g, \uparrow}$ A. Moeller, ${ }^{168, \uparrow}$ J. Nachtman, ${ }^{168, \dagger}$ H. Ogul, ${ }^{168, \text { hhhh, }, \uparrow}$ Y. Onel, ${ }^{168, \uparrow}$ F. Ozok, ${ }^{168, i i i i, \dagger}$ A. Penzo,${ }^{168, \dagger}$ C. Snyder, ${ }^{168, \dagger}$ E. Tiras, ${ }^{168, \dagger}$ J. Wetzel,${ }^{168, \dagger}$ K. Yi, ${ }^{168, j \mathrm{jjj} j, \dagger}$ O. Amram, ${ }^{169, \dagger}$ B. Blumenfeld, ${ }^{169, \dagger}$ L. Corcodilos, ${ }^{169, \dagger}$ M. Eminizer, ${ }^{169, \dagger}$ A. V. Gritsan,${ }^{169, \dagger}$ S. Kyriacou, ${ }^{169, \dagger}$ P. Maksimovic, ${ }^{169, \dagger}$ C. Mantilla, ${ }^{169, \dagger}$ J. Roskes, ${ }^{169, \dagger}$ M. Swartz, ${ }^{169, \dagger}$ T. Á. Vámi, ${ }^{169, \dagger}$ P. Baringer, ${ }^{170, \dagger}$ A. Bean, ${ }^{170, \dagger}$ A. Bylinkin, ${ }^{170, \dagger}$ S. Khalil, ${ }^{170, \dagger}$ J. King, ${ }^{170, \dagger}$ G. Krintiras,${ }^{170, \dagger}$ A. Kropivnitskaya,${ }^{170, \dagger}$ M. Murray, ${ }^{170, \dagger}$ C. Rogan,${ }^{170, \dagger}$ S. Sanders, ${ }^{170, \dagger}$ E. Schmitz, ${ }^{170, \dagger}$

J. D. Tapia Takaki, ${ }^{170, \dagger}$ Q. Wang,${ }^{170, \uparrow}$ G. Wilson, ${ }^{170, \dagger}$ S. Duric,${ }^{171, \uparrow}$ A. Ivanov, ${ }^{171, \dagger}$ K. Kaadze,${ }^{171, \dagger}$ D. Kim, ${ }^{171, \dagger}$ Y. Maravin, ${ }^{171, \dagger}$ D. R. Mendis, ${ }^{171, \dagger}$ T. Mitchell, ${ }^{171, \dagger}$ A. Modak,${ }^{171, \dagger}$ A. Mohammadi, ${ }^{171, \dagger}$ F. Rebassoo, ${ }^{172, \dagger}$ D. Wright, ${ }^{172, \uparrow}$ E. Adams, ${ }^{173, \dagger}$ A. Baden, ${ }^{173, \dagger}$ O. Baron, ${ }^{173, \dagger}$ A. Belloni, ${ }^{173, \dagger}$ S. C. Eno, ${ }^{173, \dagger}$ Y. Feng, ${ }^{173, \dagger}$ N. J. Hadley, ${ }^{173, \dagger}$ S. Jabeen, ${ }^{173, \uparrow}$ G. Y. Jeng, ${ }^{173, \dagger}$ R. G. Kellogg, ${ }^{173, \dagger}$ T. Koeth, ${ }^{173, \dagger}$ A. C. Mignerey, ${ }^{173, \dagger}$ S. Nabili, ${ }^{173, \dagger}$ M. Seidel, ${ }^{173, \dagger}$ A. Skuja, ${ }^{173, \uparrow}$ S. C. Tonwar ${ }^{173, \dagger}$ L. Wang, ${ }^{173, \dagger}$ K. Wong, ${ }^{173, \dagger}$ D. Abercrombie, ${ }^{174, \dagger}$ B. Allen, ${ }^{174, \dagger}$ R. Bi, ${ }^{174, \dagger}$ S. Brandt, ${ }^{174, \dagger}$ W. Busza, ${ }^{174, \dagger}$ I. A. Cali, ${ }^{174, \dagger}$ Y. Chen,${ }^{174, \dagger}$ M. D’Alfonso, ${ }^{174, \dagger}$ G. Gomez Ceballos, ${ }^{174, \dagger}$ M. Goncharov, ${ }^{174, \dagger}$ P. Harris, ${ }^{174, \dagger}$ D. Hsu, ${ }^{174, \uparrow}$ M. Hu, ${ }^{174, \dagger}$ M. Klute, ${ }^{174, \dagger}$ D. Kovalskyi, ${ }^{174, \dagger}$ J. Krupa, ${ }^{174, \dagger}$ Y.-J. Lee, ${ }^{174, \dagger}$ P. D. Luckey, ${ }^{174, \dagger}$ B. Maier, ${ }^{174, \dagger}$ A. C. Marini, ${ }^{174, \uparrow}$ C. Mcginn, ${ }^{174, \dagger}$ C. Mironov, ${ }^{174, \uparrow}$ S. Narayanan, ${ }^{174, \dagger}$ X. Niu, ${ }^{174, \dagger}$ C. Paus, ${ }^{174, \dagger}$ D. Rankin, ${ }^{174, \uparrow}$ C. Roland,,${ }^{174, \dagger}$ G. Roland, ${ }^{174, \uparrow}$ Z. Shi, ${ }^{174, \dagger}$ G. S. F. Stephans, ${ }^{174, \dagger}$ K. Sumorok, ${ }^{174, \dagger}$ K. Tatar, ${ }^{174, \dagger}$ D. Velicanu, ${ }^{174, \dagger}$ J. Wang,,${ }^{174, \dagger}$ T. W. Wang, ${ }^{174, \dagger}$ Z. Wang, ${ }^{174, \dagger}$ B. Wyslouch, ${ }^{174, \dagger}$ R. M. Chatterjee, ${ }^{175, \dagger}$ A. Evans, ${ }^{175, \dagger}$ S. Guts, ${ }^{175, a, \dagger}$ P. Hansen,${ }^{175, \uparrow}$ J. Hiltbrand, ${ }^{175, \dagger}$ Sh. Jain, ${ }^{175, \dagger}$ M. Krohn, ${ }^{175, \dagger}$ Y. Kubota, ${ }^{175, \dagger}$ Z. Lesko, ${ }^{175, \dagger}$ J. Mans,${ }^{175, \dagger}$ M. Revering, ${ }^{175, \dagger}$ R. Rusack, ${ }^{175, \dagger}$ R. Saradhy, ${ }^{175, \dagger}$ N. Schroeder, ${ }^{175, \uparrow}$ N. Strobbe, ${ }^{175, \dagger}$ M. A. Wadud, ${ }^{175, \dagger}$ J. G. Acosta, ${ }^{176, \uparrow}$ S. Oliveros,${ }^{176, \dagger}$ K. Bloom,${ }^{177, \dagger}$ S. Chauhan,,${ }^{177, \dagger}$ D. R. Claes,${ }^{177, \dagger}$ C. Fangmeier, ${ }^{177, \dagger}$ L. Finco, ${ }^{177, \dagger}$ F. Golf, ${ }^{177, \dagger}$ J. R. González Fernández, ${ }^{177, \dagger}$ I. Kravchenko, ${ }^{177, \dagger}$ J. E. Siado, ${ }^{177, \dagger}$ G. R. Snow, ${ }^{177, a, \dagger}$ B. Stieger,${ }^{177, \dagger}$ W. Tabb,${ }^{177, \dagger}$ G. Agarwal, ${ }^{178, \dagger}$ C. Harrington, ${ }^{178, \dagger}$ L. Hay, ${ }^{178, \dagger}$ I. Iashvili, ${ }^{178, \dagger}$ A. Kharchilava, ${ }^{178, \dagger}$ C. McLean,${ }^{178, \dagger}$ D. Nguyen, ${ }^{178, \dagger}$ A. Parker, ${ }^{178, \dagger}$ J. Pekkanen, ${ }^{178, \dagger}$ S. Rappoccio, ${ }^{178, \dagger}$ B. Roozbahani,${ }^{178, \uparrow}$ G. Alverson, ${ }^{179, \dagger}$ E. Barberis, ${ }^{179, \dagger}$ C. Freer, ${ }^{179, \dagger}$ Y. Haddad, ${ }^{179, \dagger}$ A. Hortiangtham, ${ }^{179, \dagger}$ G. Madigan, ${ }^{179, \dagger}$ B. Marzocchi, ${ }^{179, \uparrow}$ D. M. Morse ${ }^{179, \dagger}$ V. Nguyen, ${ }^{179, \dagger}$ T. Orimoto,${ }^{179, \dagger}$ L. Skinnari, ${ }^{179, \dagger}$ A. Tishelman-Charny, ${ }^{179, \dagger}$ T. Wamorkar, ${ }^{179, \dagger}$ B. Wang, ${ }^{179, \dagger}$ A. Wisecarver, ${ }^{179, \dagger}$ D. Wood,${ }^{179, \dagger}$ S. Bhattacharya, ${ }^{180, \dagger}$ J. Bueghly, ${ }^{180, \dagger}$ Z. Chen, ${ }^{180, \dagger}$ A. Gilbert, ${ }^{180, \dagger}$ 
T. Gunter, ${ }^{180, \dagger}$ K. A. Hahn, ${ }^{180, \uparrow}$ N. Odell, ${ }^{180, \dagger}$ M. H. Schmitt, ${ }^{180, \dagger}$ K. Sung,${ }^{180, \dagger}$ M. Velasco, ${ }^{180, \dagger}$ R. Bucci, ${ }^{181, \dagger}$ N. Dev, ${ }^{181, \dagger}$ R. Goldouzian, ${ }^{181, \dagger}$ M. Hildreth, ${ }^{181, \dagger}$ K. Hurtado Anampa, ${ }^{181, \dagger}$ C. Jessop, ${ }^{181, \dagger}$ D. J. Karmgard, ${ }^{181, \dagger}$ K. Lannon, ${ }^{181, \dagger}$ W. Li, ${ }^{181, \dagger}$ N. Loukas, ${ }^{181, \dagger}$ N. Marinelli, ${ }^{181, \dagger}$ I. Mcalister, ${ }^{181, \dagger}$ F. Meng,${ }^{181, \dagger}$ K. Mohrman,,${ }^{181, \dagger}$ Y. Musienko, ${ }^{181, t \mathrm{tt}, \dagger}$ R. Ruchti, ${ }^{181, \dagger}$ P. Siddireddy, ${ }^{181, \dagger}$ S. Taroni, ${ }^{181, \dagger}$ M. Wayne, ${ }^{181, \dagger}$ A. Wightman, ${ }^{181, \dagger}$ M. Wolf,${ }^{181, \dagger}$ L. Zygala, ${ }^{181, \dagger}$ J. Alimena, ${ }^{182, \dagger}$ B. Bylsma, ${ }^{182, \dagger}$ B. Cardwell, ${ }^{182, \dagger}$ L. S. Durkin, ${ }^{182, \dagger}$ B. Francis,${ }^{182, \dagger}$ C. Hill, ${ }^{182, \uparrow}$ A. Lefeld, ${ }^{182, \dagger}$ B. L. Winer, ${ }^{182, \dagger}$ B. R. Yates, ${ }^{182, \uparrow}$ G. Dezoort, ${ }^{183, \dagger}$ P. Elmer, ${ }^{183, \dagger}$ B. Greenberg, ${ }^{183, \uparrow}$ N. Haubrich, ${ }^{183, \dagger}$ S. Higginbotham, ${ }^{183, \uparrow}$ A. Kalogeropoulos, ${ }^{183, \uparrow}$ G. Kopp, ${ }^{183, \uparrow}$ S. Kwan, ${ }^{183, \uparrow}$ D. Lange, ${ }^{183, \uparrow}$ M. T. Lucchini, ${ }^{183, \uparrow}$ J. Luo, ${ }^{183, \uparrow}$ D. Marlow, ${ }^{183, \uparrow}$ K. Mei, ${ }^{183, \dagger}$ I. Ojalvo, ${ }^{183, \dagger}$ J. Olsen, ${ }^{183, \dagger}$ C. Palmer,${ }^{183, \dagger}$ P. Piroué, ${ }^{183, \dagger}$ D. Stickland, ${ }^{183, \dagger}$ C. Tully, ${ }^{183, \dagger}$ S. Malik, ${ }^{184, \dagger}$ S. Norberg, ${ }^{184, \dagger}$ V. E. Barnes, ${ }^{185, \dagger}$ R. Chawla, ${ }^{185, \dagger}$ S. Das, ${ }^{185, \dagger}$ L. Gutay, ${ }^{185, \dagger}$ M. Jones, ${ }^{185, \dagger}$ A. W. Jung, ${ }^{185, \dagger}$ B. Mahakud,${ }^{185, \uparrow}$ G. Negro, ${ }^{185, \dagger}$ N. Neumeister, ${ }^{185, \dagger}$ C. C. Peng,${ }^{185, \dagger}$ S. Piperov ${ }^{185, \dagger}$ H. Qiu ${ }^{185, \dagger}$ J. F. Schulte, ${ }^{185, \dagger}$ N. Trevisani, ${ }^{185, \dagger}$ F. Wang, ${ }^{185, \dagger}$ R. Xiao, ${ }^{185, \dagger}$ W. Xie,${ }^{185, \dagger}$ T. Cheng, ${ }^{186, \uparrow}$ J. Dolen, ${ }^{186, \dagger}$ N. Parashar, ${ }^{186, \dagger}$ M. Stojanovic, ${ }^{186, \dagger}$ A. Baty, ${ }^{187, \dagger}$ S. Dildick, ${ }^{187, \dagger}$ K. M. Ecklund, ${ }^{187, \dagger}$ S. Freed, ${ }^{187, \dagger}$ F. J. M. Geurts, ${ }^{187, \dagger}$ M. Kilpatrick, ${ }^{187, \dagger}$ A. Kumar, ${ }^{187, \dagger}$ W. Li, ${ }^{187, \dagger}$ B. P. Padley, ${ }^{187, \dagger}$ R. Redjimi, ${ }^{187, \dagger}$ J. Roberts, ${ }^{187, a, \dagger}$ J. Rorie, ${ }^{187, \dagger}$ W. Shi, ${ }^{187, \dagger}$ A. G. Stahl Leiton, ${ }^{187, \uparrow}$ A. Zhang, ${ }^{187, \dagger}$ A. Bodek, ${ }^{188, \dagger}$ P. de Barbaro, ${ }^{188, \dagger}$ R. Demina, ${ }^{188, \dagger}$ J. L. Dulemba ${ }^{188, \dagger}$ C. Fallon, ${ }^{188, \dagger}$ T. Ferbel ${ }^{188, \dagger}$ M. Galanti, ${ }^{188,}$ A. Garcia-Bellido, ${ }^{188, \dagger}$ O. Hindrichs, ${ }^{188, \dagger}$ A. Khukhunaishvili, ${ }^{18, \dagger}$ E. Ranken, ${ }^{188, \dagger}$ R. Taus, ${ }^{188, \dagger}$ R. Ciesielski, ${ }^{189, \dagger}$ B. Chiarito, ${ }^{190, \dagger}$ J. P. Chou, ${ }^{190, \dagger}$ A. Gandrakota, ${ }^{190, \dagger}$ Y. Gershtein, ${ }^{190, \dagger}$ E. Halkiadakis, ${ }^{190, \dagger}$ A. Hart, ${ }^{190, \dagger}$ M. Heindl, ${ }^{190, \dagger}$ E. Hughes ${ }^{190, \dagger}$ S. Kaplan, ${ }^{190, \dagger}$ O. Karacheban, ${ }^{190, y, \dagger}$ I. Laflotte,${ }^{190, \dagger}$ A. Lath, ${ }^{190, \dagger}$ R. Montalvo, ${ }^{190, \dagger}$ K. Nash, ${ }^{190, \dagger}$ M. Osherson, ${ }^{190, \dagger}$ S. Salur, ${ }^{190, \dagger}$ S. Schnetzer, ${ }^{190, \dagger}$ S. Somalwar, ${ }^{190, \dagger}$ R. Stone,${ }^{190, \dagger}$ S. A. Thayil, ${ }^{190, \dagger}$ S. Thomas, ${ }^{190, \uparrow}$ H. Acharya,${ }^{191, \dagger}$ A. G. Delannoy, ${ }^{191, \dagger}$ S. Spanier, ${ }^{191, \dagger}$ O. Bouhali, ${ }^{192, k k k k, \dagger}$ M. Dalchenko, ${ }^{192, \dagger}$ A. Delgado, ${ }^{192, \dagger}$ R. Eusebi,${ }^{192, \uparrow}$ J. Gilmore ${ }^{192, \dagger}$ T. Huang, ${ }^{192, \dagger}$ T. Kamon, ${ }^{192,1111, \dagger}$ H. Kim, ${ }^{192, \dagger}$ S. Luo, ${ }^{192, \dagger}$ S. Malhotra,${ }^{192, \dagger}$ R. Mueller, ${ }^{192, \dagger}$ D. Overton, ${ }^{192, \dagger}$ L. Perniè ${ }^{192, \dagger}$ D. Rathjens, ${ }^{192, \dagger}$ A. Safonov, ${ }^{192, \dagger}$ J. Sturdy, ${ }^{192, \dagger}$ N. Akchurin, ${ }^{193, \dagger}$ J. Damgov, ${ }^{193, \dagger}$ V. Hegde, ${ }^{193, \dagger}$ S. Kunori, ${ }^{193, \dagger}$ K. Lamichhane, ${ }^{193, \dagger}$ S. W. Lee, ${ }^{193, \dagger}$ T. Mengke, ${ }^{193, \dagger}$ S. Muthumuni, ${ }^{193, \dagger}$ T. Peltola,${ }^{193, \dagger}$ S. Undleeb, ${ }^{193, \dagger}$ I. Volobouev, ${ }^{193, \uparrow}$ Z. Wang,${ }^{193, \dagger}$ A. Whitbeck, ${ }^{193, \dagger}$ E. Appelt, ${ }^{194, \dagger}$ S. Greene, ${ }^{194, \dagger}$ A. Gurrola,${ }^{194, \dagger}$ R. Janjam, ${ }^{194, \dagger}$ W. Johns, ${ }^{194, \dagger}$ C. Maguire,${ }^{194, \dagger}$ A. Melo, ${ }^{194, \dagger}$ H. Ni,${ }^{194, \dagger}$ K. Padeken, ${ }^{194, \dagger}$ F. Romeo, ${ }^{194, \dagger}$ P. Sheldon, ${ }^{194, \dagger}$ S. Tuo, ${ }^{194, \dagger}$ J. Velkovska,${ }^{194, \dagger}$ M. Verweij,${ }^{194, \dagger}$

L. Ang, ${ }^{195, \dagger}$ M. W. Arenton, ${ }^{195, \dagger}$ B. Cox,${ }^{195, \dagger}$ G. Cummings,${ }^{195, \dagger}$ J. Hakala, ${ }^{195, \dagger}$ R. Hirosky, ${ }^{195, \dagger}$ M. Joyce,${ }^{195, \dagger}$ A. Ledovskoy, ${ }^{195, \dagger}$ C. Neu, ${ }^{195, \dagger}$ B. Tannenwald, ${ }^{195, \dagger}$ Y. Wang, ${ }^{195, \dagger}$ E. Wolfe, ${ }^{195, \dagger}$ F. Xia, ${ }^{195, \dagger}$ P. E. Karchin, ${ }^{196, \dagger}$ N. Poudyal, ${ }^{196, \dagger}$ P. Thapa, ${ }^{196, \dagger}$ K. Black, ${ }^{197, \dagger}$ T. Bose, ${ }^{197, \dagger}$ J. Buchanan, ${ }^{197, \dagger}$ C. Caillol,,${ }^{197, \dagger}$ S. Dasu, ${ }^{197, \dagger}$ I. De Bruyn, ${ }^{197, \uparrow}$ C. Galloni, ${ }^{197, \dagger}$ H. He,${ }^{197, \dagger}$ M. Herndon, ${ }^{197, \dagger}$ A. Hervé, ${ }^{197, \dagger}$ U. Hussain,${ }^{197, \dagger}$ A. Lanaro, ${ }^{197, \dagger}$ A. Loeliger, ${ }^{197, \dagger}$ R. Loveless, ${ }^{197, \dagger}$

J. Madhusudanan Sreekala, ${ }^{197, \dagger}$ A. Mallampalli, ${ }^{197, \dagger}$ D. Pinna,${ }^{197, \dagger}$ T. Ruggles, ${ }^{197, \dagger}$ A. Savin, ${ }^{197, \dagger}$ V. Shang, ${ }^{197, \dagger}$ V. Sharma ${ }^{197, \dagger}$ W. H. Smith ${ }^{197, \dagger}$ D. Teague,${ }^{197, \dagger}$ S. Trembath-reichert, ${ }^{197, \dagger}$ W. Vetens,${ }^{197, \dagger}$ G. Antchev, ${ }^{209, \sharp}$ P. Aspell, ${ }^{206, \sharp}$ I. Atanassov, ${ }^{209, \sharp}$ V. Avati, ${ }^{204,206, \ddagger}$ J. Baechler, ${ }^{206, \$}$ C. Baldenegro Barrera, ${ }^{208, \sharp}$ V. Berardi, ${ }^{201 \mathrm{a}, 201 \mathrm{~b}, \sharp}$ M. Berretti, ${ }^{199 \mathrm{a}, \sharp}$

V. Borchsh, ${ }^{205, \$}$ E. Bossini, ${ }^{206,203 \mathrm{~b}, \sharp}$ U. Bottigli, ${ }^{203 \mathrm{c}, \sharp}$ M. Bozzo, ${ }^{202 \mathrm{a}, 202 \mathrm{~b}, \sharp}$ H. Burkhardt, ${ }^{206, \$}$ F. S. Cafagna, ${ }^{201 a, \sharp}$

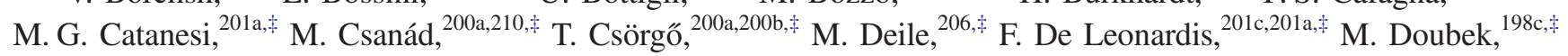

D. Druzhkin, ${ }^{205,206, \sharp}$ K. Eggert, ${ }^{207, \$}$ V. Eremin, ${ }^{212, \$}$ A. Fiergolski, ${ }^{206, \$}$ L. Forthomme, ${ }^{199 a, 199 b, \ddagger}$ F. Garcia, ${ }^{199 a, \sharp}$

V. Georgiev, ${ }^{198 \mathrm{a}, \sharp}$ S. Giani,${ }^{206,}$ L. Grzanka, ${ }^{204, \$}$ J. Hammerbauer, ${ }^{198 a, \sharp}$ T. Isidori, ${ }^{208, \sharp}$ V. Ivanchenko, ${ }^{205, \$}$ M. Janda, ${ }^{198 c, \$}$

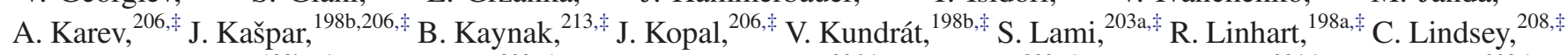
M. V. Lokajíček,${ }^{198 b, a, \sharp}$ L. Losurdo ${ }^{203 c, \$}$ F. Lucas Rodríguez, ${ }^{206, \$}$ M. Macrí, ${ }^{202 a, \$}$ M. Malawski, ${ }^{204, \$}$ N. Minafra, ${ }^{208, \sharp}$ S. Minutoli ${ }^{202 a, \$}$ T. Naaranoja, ${ }^{199 a, 199 b, \$}$ F. Nemes,${ }^{206,200 a, \sharp}$ H. Niewiadomski, ${ }^{207, \sharp}$ T. Novák, ${ }^{200 b, \sharp}$ E. Oliveri, ${ }^{206, \sharp}$ F. Oljemark, ${ }^{199 \mathrm{a}, 199 \mathrm{~b}, \$}$ M. Oriunno, ${ }^{214, \$}$ K. Österberg, ${ }^{199 \mathrm{a}, 199 \mathrm{~b}, \$}$ P. Palazzi, ${ }^{206, \$}$ V. Passaro, ${ }^{201 \mathrm{c}, 201 \mathrm{a}, \$} \mathrm{Z}$. Peroutka, ${ }^{198 \mathrm{a}, \$}$ J. Procházka, ${ }^{198 b, \sharp}$ M. Quinto, ${ }^{201 \mathrm{a}, 201 \mathrm{~b}, \$}$ E. Radermacher, ${ }^{206, \$}$ E. Radicioni, ${ }^{201 \mathrm{a}, \$}$ F. Ravotti, ${ }^{206, \$}$ C. Royon, ${ }^{208, \$}$ G. Ruggiero, ${ }^{206, \ddagger}$ H. Saarikko, ${ }^{199 a, 199 b, \sharp}$ V. D. Samoylenko, ${ }^{211, \sharp}$ A. Scribano, ${ }^{203 a, \not}$ J. Široký, ${ }^{198 a, \sharp}$ J. Smajek, ${ }^{206, \sharp}$ W. Snoeys, ${ }^{206,}$ R. Stefanovitch, ${ }^{206, \$}$ J. Sziklai, ${ }^{200 a, \$}$ C. Taylor, ${ }^{207, \$}$ E. Tcherniaev, ${ }^{205,}$ N. Turini, ${ }^{203 c, \$}$ O. Urban, ${ }^{198 a, \$}$ V. Vacek, ${ }^{198 c, \$}$ O. Vavroch ${ }^{198 \mathrm{a}, \$}$ J. Welti, ${ }^{199 \mathrm{a}, 199 \mathrm{~b}, \$}$ J. Williams, ${ }^{208, \$}$ J. Zich, ${ }^{198 \mathrm{a}, \$}$ and K. Zielinski ${ }^{204, \$}$

(CMS Collaboration $)^{\dagger}$

(TOTEM Collaboration) $^{\ddagger}$

${ }^{1}$ Yerevan Physics Institute, Yerevan, Armenia

${ }^{2}$ Institut für Hochenergiephysik, Wien, Austria 


\author{
${ }^{3}$ Institute for Nuclear Problems, Minsk, Belarus \\ ${ }^{4}$ Universiteit Antwerpen, Antwerpen, Belgium \\ ${ }^{5}$ Vrije Universiteit Brussel, Brussel, Belgium \\ ${ }^{6}$ Université Libre de Bruxelles, Bruxelles, Belgium \\ ${ }^{7}$ Ghent University, Ghent, Belgium \\ ${ }^{8}$ Université Catholique de Louvain, Louvain-la-Neuve, Belgium \\ ${ }^{9}$ Centro Brasileiro de Pesquisas Fisicas, Rio de Janeiro, Brazil \\ ${ }^{10}$ Universidade do Estado do Rio de Janeiro, Rio de Janeiro, Brazil \\ ${ }^{11 a}$ Universidade Estadual Paulista, São Paulo, Brazil \\ ${ }^{11 \mathrm{~b}}$ Universidade Federal do ABC, São Paulo, Brazil \\ ${ }^{12}$ Institute for Nuclear Research and Nuclear Energy, Bulgarian Academy of Sciences, Sofia, Bulgaria \\ ${ }^{13}$ University of Sofia, Sofia, Bulgaria \\ ${ }^{14}$ Beihang University, Beijing, China \\ ${ }^{15}$ Department of Physics, Tsinghua University, Beijing, China \\ ${ }^{16}$ Institute of High Energy Physics, Beijing, China \\ ${ }^{17}$ State Key Laboratory of Nuclear Physics and Technology, Peking University, Beijing, China \\ ${ }^{18}$ Sun Yat-Sen University, Guangzhou, China \\ ${ }^{19}$ Institute of Modern Physics and Key Laboratory of Nuclear Physics and Ion-beam Application \\ (MOE)_Fudan University, Shanghai, China \\ ${ }^{20}$ Zhejiang University, Hangzhou, China \\ ${ }^{21}$ Universidad de Los Andes, Bogota, Colombia \\ ${ }^{22}$ Universidad de Antioquia, Medellin, Colombia \\ ${ }^{23}$ University of Split, Faculty of Electrical Engineering, Mechanical Engineering and Naval Architecture, \\ Split, Croatia \\ ${ }^{24}$ University of Split, Faculty of Science, Split, Croatia \\ ${ }^{25}$ Institute Rudjer Boskovic, Zagreb, Croatia \\ ${ }^{26}$ University of Cyprus, Nicosia, Cyprus \\ ${ }^{27}$ Charles University, Prague, Czech Republic \\ ${ }^{28}$ Escuela Politecnica Nacional, Quito, Ecuador \\ ${ }^{29}$ Universidad San Francisco de Quito, Quito, Ecuador \\ ${ }^{30}$ Academy of Scientific Research and Technology of the Arab Republic of Egypt, \\ Egyptian Network of High Energy Physics, Cairo, Egypt \\ ${ }^{31}$ Center for High Energy Physics (CHEP-FU), Fayoum University, El-Fayoum, Egypt \\ ${ }^{32}$ National Institute of Chemical Physics and Biophysics, Tallinn, Estonia \\ ${ }^{33}$ Department of Physics, University of Helsinki, Helsinki, Finland \\ ${ }^{34}$ Helsinki Institute of Physics, Helsinki, Finland \\ ${ }^{35}$ Lappeenranta University of Technology, Lappeenranta, Finland \\ ${ }^{36}$ IRFU, CEA, Université Paris-Saclay, Gif-sur-Yvette, France \\ ${ }^{37}$ Laboratoire Leprince-Ringuet, CNRS/IN2P3, Ecole Polytechnique, Institut Polytechnique de Paris, \\ Palaiseau, France \\ ${ }^{38}$ Université de Strasbourg, CNRS, IPHC UMR 7178, Strasbourg, France \\ ${ }^{39}$ Université de Lyon, Université Claude Bernard Lyon 1, CNRS-IN2P3, \\ Institut de Physique Nucléaire de Lyon, Villeurbanne, France \\ ${ }^{40}$ Georgian Technical University, Tbilisi, Georgia \\ ${ }^{41}$ RWTH Aachen University, I. Physikalisches Institut, Aachen, Germany \\ ${ }^{42}$ RWTH Aachen University, III. Physikalisches Institut A, Aachen, Germany \\ ${ }^{43}$ RWTH Aachen University, III. Physikalisches Institut B, Aachen, Germany \\ ${ }^{44}$ Deutsches Elektronen-Synchrotron, Hamburg, Germany \\ ${ }^{45}$ University of Hamburg, Hamburg, Germany \\ ${ }^{46}$ Karlsruher Institut fuer Technologie, Karlsruhe, Germany \\ ${ }^{47}$ Institute of Nuclear and Particle Physics (INPP), NCSR Demokritos, Aghia Paraskevi, Greece \\ ${ }^{48}$ National and Kapodistrian University of Athens, Athens, Greece \\ ${ }^{49}$ National Technical University of Athens, Athens, Greece \\ ${ }^{50}$ University of Ioánnina, Ioánnina, Greece \\ ${ }^{51}$ MTA-ELTE Lendület CMS Particle and Nuclear Physics Group, Eötvös Loránd University, \\ Budapest, Hungary \\ ${ }^{52}$ Wigner Research Centre for Physics, Budapest, Hungary \\ ${ }^{53}$ Institute of Nuclear Research ATOMKI, Debrecen, Hungary \\ ${ }^{54}$ Institute of Physics, University of Debrecen, Debrecen, Hungary \\ ${ }^{55}$ Eszterhazy Karoly University, Karoly Robert Campus, Gyongyos, Hungary
}




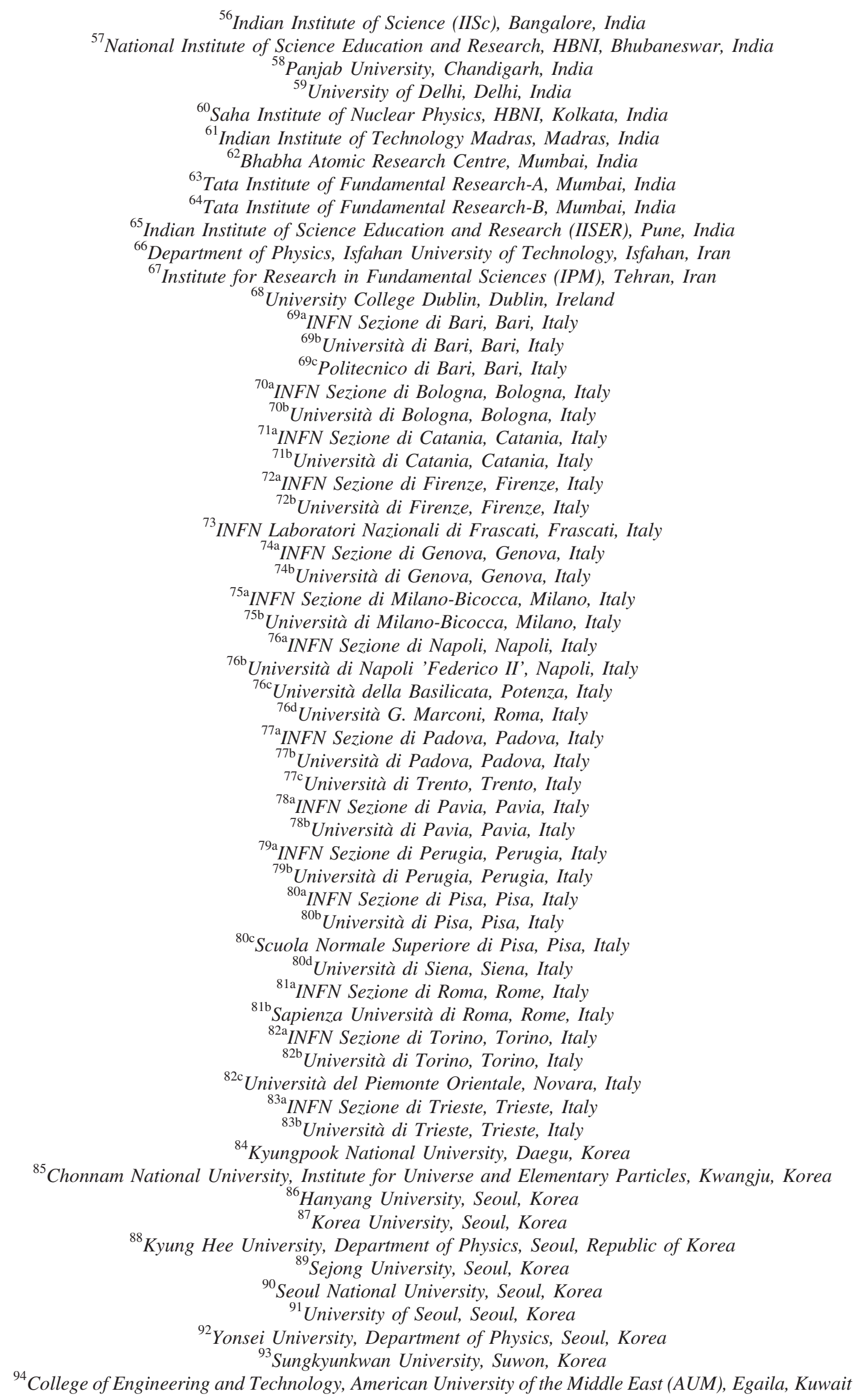




\author{
${ }^{95}$ Riga Technical University, Riga, Latvia \\ ${ }^{96}$ Vilnius University, Vilnius, Lithuania \\ ${ }^{97}$ National Centre for Particle Physics, Universiti Malaya, Kuala Lumpur, Malaysia \\ ${ }^{98}$ Universidad de Sonora (UNISON), Hermosillo, Mexico \\ ${ }^{99}$ Centro de Investigacion y de Estudios Avanzados del IPN, Mexico City, Mexico \\ ${ }^{100}$ Universidad Iberoamericana, Mexico City, Mexico \\ ${ }^{101}$ Benemerita Universidad Autonoma de Puebla, Puebla, Mexico \\ ${ }^{102}$ Universidad Autónoma de San Luis Potosí, San Luis Potosí, Mexico \\ ${ }^{103}$ University of Montenegro, Podgorica, Montenegro \\ ${ }^{104}$ University of Auckland, Auckland, New Zealand \\ ${ }^{105}$ University of Canterbury, Christchurch, New Zealand \\ ${ }^{106}$ National Centre for Physics, Quaid-I-Azam University, Islamabad, Pakistan \\ ${ }^{107}$ AGH University of Science and Technology Faculty of Computer Science, Electronics and \\ Telecommunications, Krakow, Poland \\ ${ }^{108}$ National Centre for Nuclear Research, Swierk, Poland \\ ${ }^{109}$ Institute of Experimental Physics, Faculty of Physics, University of Warsaw, Warsaw, Poland \\ ${ }^{110}$ Laboratório de Instrumentação e Física Experimental de Partículas, Lisboa, Portugal \\ ${ }^{111}$ Joint Institute for Nuclear Research, Dubna, Russia \\ ${ }^{112}$ Petersburg Nuclear Physics Institute, Gatchina (St. Petersburg), Russia \\ ${ }^{113}$ Institute for Nuclear Research, Moscow, Russia \\ ${ }^{114}$ Institute for Theoretical and Experimental Physics named by A.I. Alikhanov of NRC \\ 'Kurchatov Institute', Moscow, Russia \\ ${ }^{115}$ Moscow Institute of Physics and Technology, Moscow, Russia \\ ${ }^{116}$ National Research Nuclear University 'Moscow Engineering Physics Institute' (MEPhI), \\ Moscow, Russia \\ ${ }^{117}$ P.N. Lebedev Physical Institute, Moscow, Russia \\ ${ }^{118}$ Skobeltsyn Institute of Nuclear Physics, Lomonosov Moscow State University, Moscow, Russia \\ ${ }^{119}$ Novosibirsk State University (NSU), Novosibirsk, Russia \\ ${ }^{120}$ Institute for High Energy Physics of National Research Centre 'Kurchatov Institute', Protvino, Russia \\ ${ }^{121}$ National Research Tomsk Polytechnic University, Tomsk, Russia \\ ${ }^{122}$ Tomsk State University, Tomsk, Russia \\ ${ }^{123}$ University of Belgrade: Faculty of Physics and VINCA Institute of Nuclear Sciences, Belgrade, Serbia \\ ${ }^{124}$ Centro de Investigaciones Energéticas Medioambientales y Tecnológicas (CIEMAT), Madrid, Spain \\ ${ }^{125}$ Universidad Autónoma de Madrid, Madrid, Spain \\ ${ }^{126}$ Universidad de Oviedo, Instituto Universitario de Ciencias y Tecnologías Espaciales de Asturias \\ (ICTEA), Oviedo, Spain \\ ${ }^{127}$ Instituto de Física de Cantabria (IFCA), CSIC-Universidad de Cantabria, Santander, Spain \\ ${ }^{128}$ University of Colombo, Colombo, Sri Lanka \\ ${ }^{129}$ University of Ruhuna, Department of Physics, Matara, Sri Lanka \\ ${ }^{130}$ CERN, European Organization for Nuclear Research, Geneva, Switzerland \\ ${ }^{131}$ Paul Scherrer Institut, Villigen, Switzerland \\ ${ }^{132}$ ETH Zurich-Institute for Particle Physics and Astrophysics (IPA), Zurich, Switzerland \\ ${ }^{133}$ Universität Zürich, Zurich, Switzerland \\ ${ }^{134}$ National Central University, Chung-Li, Taiwan \\ ${ }^{135}$ National Taiwan University (NTU), Taipei, Taiwan \\ ${ }^{136}$ Chulalongkorn University, Faculty of Science, Department of Physics, Bangkok, Thailand \\ ${ }^{137}$ Çukurova University, Physics Department, Science and Art Faculty, Adana, Turkey \\ ${ }^{138}$ Middle East Technical University, Physics Department, Ankara, Turkey \\ ${ }^{139}$ Bogazici University, Istanbul, Turkey \\ ${ }^{140}$ Istanbul Technical University, Istanbul, Turkey \\ ${ }^{141}$ Istanbul U., Istanbul, Turkey \\ ${ }^{142}$ Institute for Scintillation Materials of National Academy of Science of Ukraine, Kharkov, Ukraine \\ ${ }^{143}$ National Scientific Center, Kharkov Institute of Physics and Technology, Kharkov, Ukraine \\ ${ }^{144}$ University of Bristol, Bristol, United Kingdom \\ ${ }^{145}$ Rutherford Appleton Laboratory, Didcot, United Kingdom \\ ${ }^{146}$ Imperial College, London, United Kingdom \\ ${ }^{147}$ Brunel University, Uxbridge, United Kingdom \\ ${ }^{148}$ Baylor University, Waco, Texas, USA \\ ${ }^{149}$ Catholic University of America, Washington, DC, USA \\ ${ }^{150}$ The University of Alabama, Tuscaloosa, Alabama, USA
}




\author{
${ }^{151}$ Boston University, Boston, Massachusetts, USA \\ ${ }^{152}$ Brown University, Providence, Rhode Island, USA \\ ${ }^{153}$ University of California, Davis, Davis, California, USA \\ ${ }^{154}$ University of California, Los Angeles, California, USA \\ ${ }^{155}$ University of California, Riverside, Riverside, California, USA \\ ${ }^{156}$ University of California, San Diego, La Jolla, California, USA \\ ${ }^{157}$ University of California, Santa Barbara-Department of Physics, Santa Barbara, California, USA \\ ${ }^{158}$ California Institute of Technology, Pasadena, California, USA \\ ${ }^{159}$ Carnegie Mellon University, Pittsburgh, Pennsylvania, USA \\ ${ }^{160}$ University of Colorado Boulder, Boulder, Colorado, USA \\ ${ }^{161}$ Cornell University, Ithaca, New York, USA \\ ${ }^{162}$ Fermi National Accelerator Laboratory, Batavia, Illinois, USA \\ ${ }^{163}$ University of Florida, Gainesville, Florida, USA \\ ${ }^{164}$ Florida International University, Miami, Florida, USA \\ ${ }^{165}$ Florida State University, Tallahassee, Florida, USA \\ ${ }^{166}$ Florida Institute of Technology, Melbourne, Florida, USA \\ ${ }^{167}$ University of Illinois at Chicago (UIC), Chicago, Illinois, USA \\ ${ }^{168}$ The University of Iowa, Iowa City, Iowa, USA \\ ${ }^{169}$ Johns Hopkins University, Baltimore, Maryland, USA \\ ${ }^{170}$ The University of Kansas, Lawrence, Kansas, USA \\ ${ }^{171}$ Kansas State University, Manhattan, Kansas, USA \\ ${ }^{172}$ Lawrence Livermore National Laboratory, Livermore, California, USA \\ ${ }^{173}$ University of Maryland, College Park, Maryland, USA \\ ${ }^{174}$ Massachusetts Institute of Technology, Cambridge, Massachusetts, USA \\ ${ }^{175}$ University of Minnesota, Minneapolis, Minnesota, USA \\ ${ }^{176}$ University of Mississippi, Oxford, Mississippi, USA \\ ${ }^{177}$ University of Nebraska-Lincoln, Lincoln, Nebraska, USA \\ ${ }^{178}$ State University of New York at Buffalo, Buffalo, New York, USA \\ ${ }^{179}$ Northeastern University, Boston, Massachusetts, USA \\ ${ }^{180}$ Northwestern University, Evanston, Illinois, USA \\ ${ }^{181}$ University of Notre Dame, Notre Dame, Indiana, USA \\ ${ }^{182}$ The Ohio State University, Columbus, Ohio, USA \\ ${ }^{183}$ Princeton University, Princeton, New Jersey, USA \\ ${ }^{184}$ University of Puerto Rico, Mayaguez, Puerto Rico, USA \\ ${ }^{185}$ Purdue University, West Lafayette, Indiana, USA \\ ${ }^{186}$ Purdue University Northwest, Hammond, Indiana, USA \\ ${ }^{187}$ Rice University, Houston, Texas, USA \\ ${ }^{188}$ University of Rochester, Rochester, New York, USA \\ ${ }^{189}$ The Rockefeller University, New York, New York, USA \\ ${ }^{190}$ Rutgers, The State University of New Jersey, Piscataway, New Jersey, USA \\ ${ }^{191}$ University of Tennessee, Knoxville, Tennessee, USA \\ ${ }^{192}$ Texas A\&M University, College Station, Texas, USA \\ ${ }^{193}$ Texas Tech University, Lubbock, Texas, USA \\ ${ }^{194}$ Vanderbilt University, Nashville, Tennessee, USA \\ ${ }^{195}$ University of Virginia, Charlottesville, Virginia, USA \\ ${ }^{196}$ Wayne State University, Detroit, Michigan, USA \\ ${ }^{197}$ University of Wisconsin-Madison, Madison, WI, Wisconsin, USA \\ ${ }^{198 a}$ University of West Bohemia, Pilsen, Czech Republic \\ ${ }^{198 \mathrm{~b}}$ Institute of Physics of the Academy of Sciences of the Czech Republic, Prague, Czech Republic \\ ${ }^{198 \mathrm{c}}$ Czech Technical University, Prague, Czech Republic \\ ${ }^{199 a}$ Helsinki Institute of Physics, University of Helsinki, Helsinki, Finland \\ ${ }^{199 \mathrm{~b}}$ Department of Physics, University of Helsinki, Helsinki, Finland \\ ${ }^{200 a}$ Wigner Research Centre for Physics, RMKI, Budapest, Hungary \\ ${ }^{200 \mathrm{~b}}$ Eszterhazy Karoly University KRC, Gyöngyös, Hungary \\ ${ }^{201 a}$ INFN Sezione di Bari, Bari, Italy \\ ${ }^{201 \mathrm{~b}}$ Dipartimento Interateneo di Fisica di Bari, University of Bari, Bari, Italy \\ ${ }^{201 \mathrm{c}}$ Dipartimento di Ingegneria Elettrica e dell'Informazione-Politecnico di Bari, Bari, Italy \\ ${ }^{202 a}$ INFN Sezione di Genova, Genova, Italy \\ ${ }^{202 \mathrm{~b}}$ Università degli Studi di Genova, Genova, Italy \\ ${ }^{203 a}$ INFN Sezione di Pisa, Pisa, Italy
}




\author{
${ }^{203 b}$ Università degli Studi di Pisa, Pisa, Italy \\ ${ }^{203 c}$ Università degli Studi di Siena and Gruppo Collegato INFN di Siena, Siena, Italy \\ ${ }^{204}$ Akademia Górniczo-Hutnicza (AGH) University of Science and Technology, Krakow, Poland \\ ${ }^{205}$ Tomsk State University, Tomsk, Russia \\ ${ }^{206}$ CERN, Geneva, Switzerland \\ ${ }^{207}$ Department of Physics, Case Western Reserve University, Cleveland, Ohio, USA \\ ${ }^{208}$ The University of Kansas, Lawrence, Kansas, USA \\ ${ }^{209}$ INRNE-BAS, Institute for Nuclear Research and Nuclear Energy, Bulgarian Academy of Sciences, \\ Sofia, Bulgaria \\ ${ }^{210}$ Department of Atomic Physics, Eötvös Loránd University, Budapest, Hungary \\ ${ }^{211}$ NRC 'Kurchatov Institute'-IHEP, Protvino, Russia \\ ${ }^{212}$ Ioffe Phys. Tech. Inst., Russian Academy of Sciences, St. Petersburg, Russia \\ ${ }^{213}$ Istanbul U., Istanbul, Turkey \\ ${ }^{214}$ SLAC, Palo Alto, California, USA
}

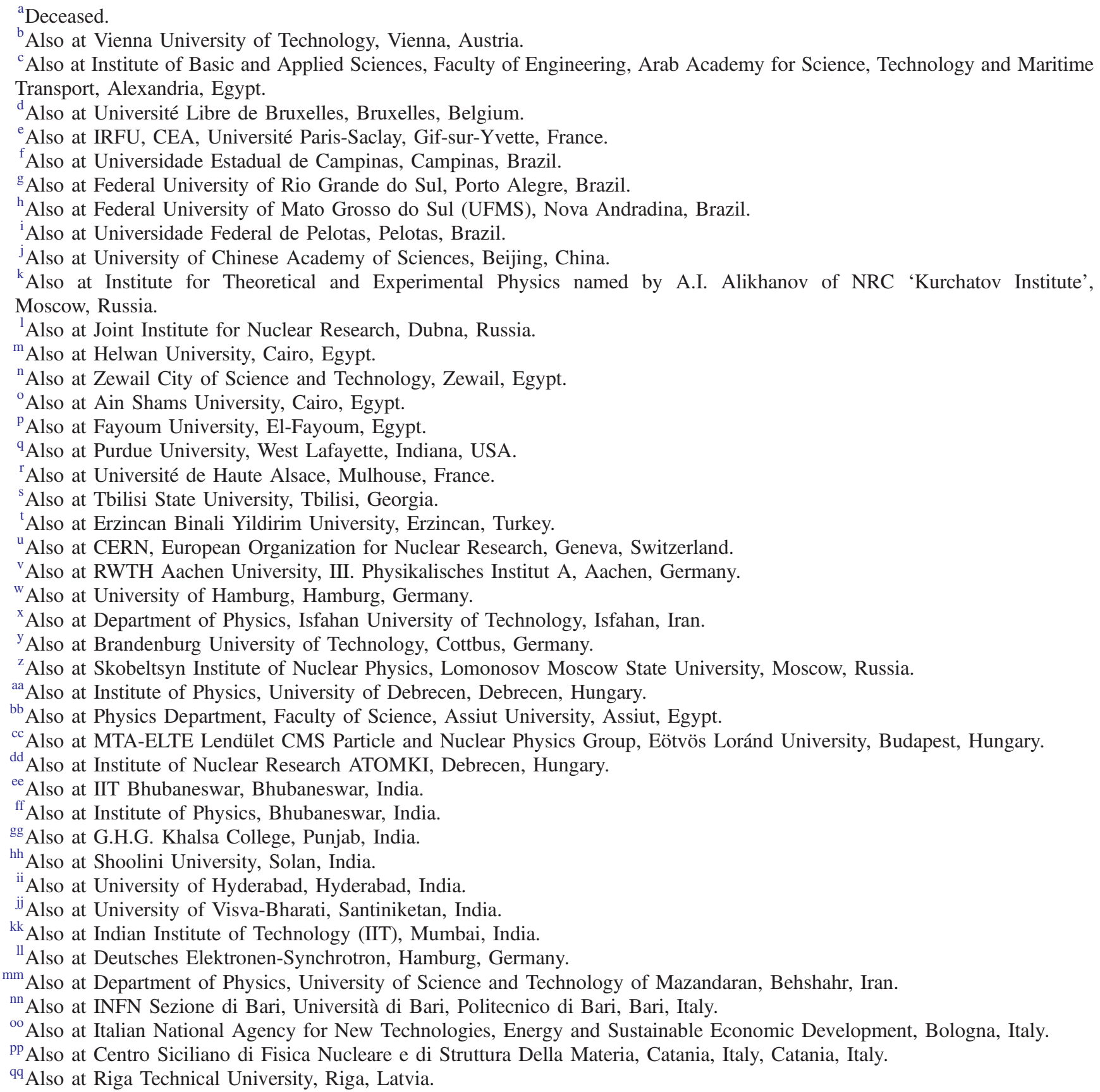


${ }^{r r}$ Also at Consejo Nacional de Ciencia y Tecnología, Mexico City, Mexico.

${ }^{\text {ss }}$ Also at Warsaw University of Technology, Institute of Electronic Systems, Warsaw, Poland.

${ }^{t t}$ Also at Institute for Nuclear Research, Moscow, Russia.

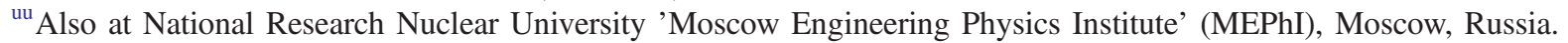

${ }^{\mathrm{v}}$ Also at St. Petersburg State Polytechnical University, St. Petersburg, Russia.

${ }^{w w}$ Also at University of Florida, Gainesville, Florida, USA.

${ }^{\mathrm{xx}}$ Also at Imperial College, London, United Kingdom.

${ }^{\text {yy }}$ Also at P.N. Lebedev Physical Institute, Moscow, Russia.

${ }^{\mathrm{zz}}$ Also at Budker Institute of Nuclear Physics, Novosibirsk, Russia.

${ }^{\text {aaa }}$ Also at Faculty of Physics, University of Belgrade, Belgrade, Serbia.

${ }^{b b b}$ Also at Università degli Studi di Siena, Siena, Italy.

${ }^{c c c}$ Also at Trincomalee Campus, Eastern University, Sri Lanka.

${ }^{\text {ddd }}$ Also at INFN Sezione di Pavia, Università di Pavia, Pavia, Italy.

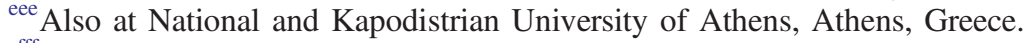

${ }^{\mathrm{fff}}$ Also at Universität Zürich, Zurich, Switzerland.

${ }^{\mathrm{ggg}}$ Also at Stefan Meyer Institute for Subatomic Physics, Vienna, Austria.

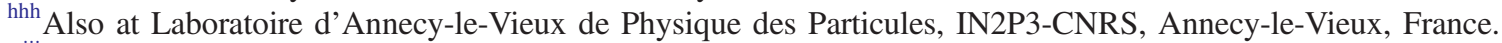

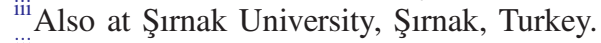

${ }^{\mathrm{jjj}}$ Also at Department of Physics, Tsinghua University, Beijing, China.

${ }^{k k k}$ Also at Near East University, Research Center of Experimental Health Science, Nicosia, Turkey.

${ }^{111}$ Also at Beykent University, Istanbul, Turkey.

${ }^{\mathrm{mmm}}$ Also at Istanbul Aydin University, Application and Research Center for Advanced Studies (App. \& Res. Cent. for Advanced Studies), Istanbul, Turkey.

${ }^{\mathrm{nnn}}$ Also at Mersin University, Mersin, Turkey.

${ }^{\circ o o}$ Also at Piri Reis University, Istanbul, Turkey.

${ }^{\mathrm{ppp}}$ Also at Adiyaman University, Adiyaman, Turkey.

${ }^{\mathrm{qqq}}$ Also at Ozyegin University, Istanbul, Turkey.

${ }^{\text {rrr }}$ Also at Izmir Institute of Technology, Izmir, Turkey.

${ }^{\text {sss }}$ Also at Necmettin Erbakan University, Konya, Turkey.

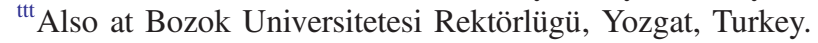

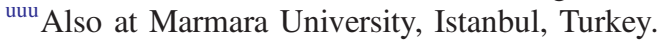

${ }^{\mathrm{vvv}}$ Also at Milli Savunma University, Istanbul, Turkey.

${ }^{\text {www }}$ Also at Kafkas University, Kars, Turkey.

${ }^{\mathrm{xxx}}$ Also at Istanbul Bilgi University, Istanbul, Turkey.

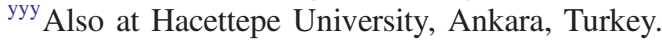

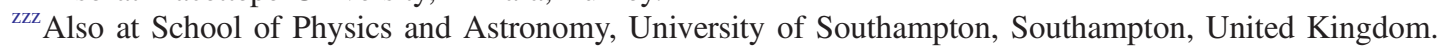

${ }^{\text {aaa }}$ Also at IPPP Durham University, Durham, United Kingdom.

${ }^{b b b b}$ Also at Monash University, Faculty of Science, Clayton, Australia.

${ }^{\mathrm{cccc}}$ Also at Bethel University, St. Paul, Minneapolis, USA.

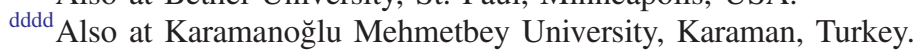

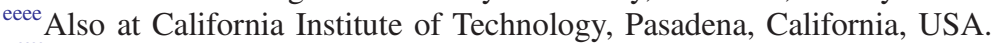

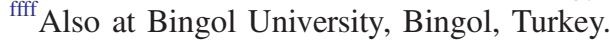

${ }^{\text {gggg }}$ Also at Georgian Technical University, Tbilisi, Georgia.

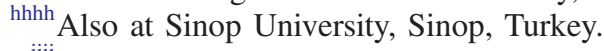

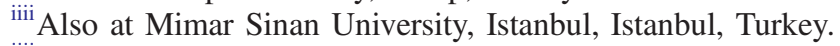

${ }^{j j j j}$ Also at Nanjing Normal University Department of Physics, Nanjing, China.

${ }^{k k k k}$ Also at Texas A\&M University at Qatar, Doha, Qatar.

${ }^{1111}$ Also at Kyungpook National University, Daegu, Korea. 